\title{
Microwave-Assisted Synthesis of Phenanthridines by Radical Insertion/Cyclization of Biphenyl Isocyanides
}

\author{
Yulong Xu, ${ }^{\dagger}$ Yiyi Chen ${ }^{\dagger}$ Wei $\mathrm{Li},{ }^{\dagger}$ Qiong Xie, ${ }^{\dagger}$ and Liming Shao ${ }^{*}, \dagger$ \\ ${ }^{\dagger}$ School of Pharmacy, Fudan University, 826 Zhangheng Road, Zhangjiang Hi-tech \\ Park, Pudong, Shanghai 201203, China \\ ${ }^{\ddagger}$ Shanghai Center for Drug Discovery \& Development, 826 Zhangheng Road, \\ Zhangjiang Hi-tech Park, Pudong, Shanghai 201203, China \\ *E-mail for L. Shao: limingshao@fudan.edu.cn.
}

\section{Supporting Information}

I . General Information 2

II. Kinetic Isotope Effect Studies 3-5

III. Radical Inhibition Studies 6-7

IV. ${ }^{1} \mathrm{H}$ and ${ }^{13} \mathrm{C}$ NMR $\quad 8-50$ 


\section{Supporting Information}

\section{General Information}

All reactions were carried out under an argon atmosphere. All commercially available reagents were used without further purification unless otherwise stated. All of the microwave-assisted reactions were performed in an Initiator+ microwave system at the specified temperature using the standard mode of operation. The reactions were monitored by thin-layer chromatography (TLC) analysis. The silica gel (200-300 mesh) was used for column chromatography. High-resolution MS (HRMS) was analyzed by a TOF analyzer. The ion source is electrospray ionization (ESI). ${ }^{1} \mathrm{H}$ and ${ }^{13} \mathrm{C}$ spectra were recorded at $400 \mathrm{MHz}$ and $600 \mathrm{MHz}$, respectively. Chemical shifts in ${ }^{1} \mathrm{H}$ NMR spectra are reported in parts per million (ppm) on the $\delta$ scale from an internal standard of $\mathrm{CDCl}_{3}$ (7.26 ppm). Data are reported as follows: chemical shift $(\delta \mathrm{ppm})$, multiplicity $(\mathrm{s}=$ singlet, $\mathrm{d}=$ doublet, $\mathrm{t}=$ triplet, $\mathrm{q}=$ quartet, $\mathrm{m}=$ multiplet, $\mathrm{br}=\mathrm{Broad})$, coupling constant in hertz $(\mathrm{Hz})$, and integration. Chemical shifts of ${ }^{13} \mathrm{C}$ NMR spectra are reported in ppm from the central peak of $\mathrm{CDCl}_{3}(77.0 \mathrm{ppm})$ on the $\delta$ scale. 


\section{Kinetic Isotope Effect Studies}

Kinetic Isotope Effect Study between $2 \mathrm{c}$ and $\left[\mathrm{D}_{8}\right]-2 \mathrm{c}$

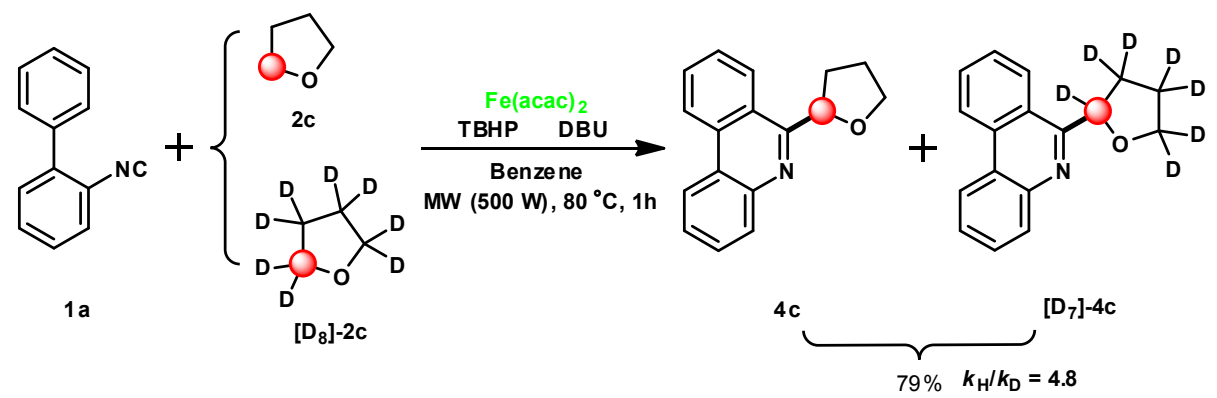

$k_{\mathrm{H}} / k_{\mathrm{D}}=0.83 / 0.17=4.8 / 1$. (The KIE was determined by ${ }^{1} \mathrm{H}$ NMR spectroscopy by analyzing the ratio of $4 \mathbf{c}$ and $\left.\left[\mathbf{D}_{7}\right]-\mathbf{4} \mathbf{c}\right)$.

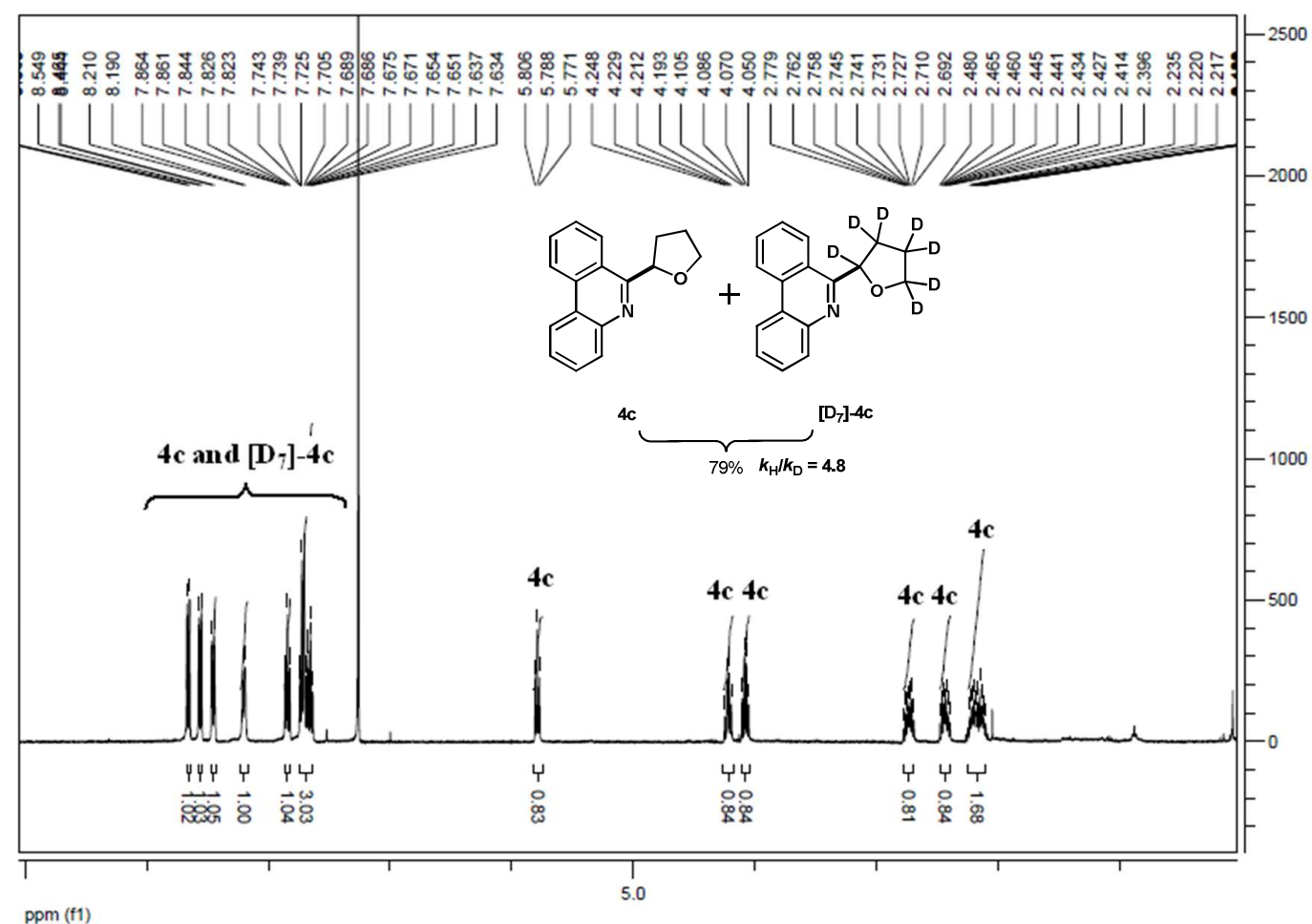


Intermolecular Kinetic Isotope Effect Study

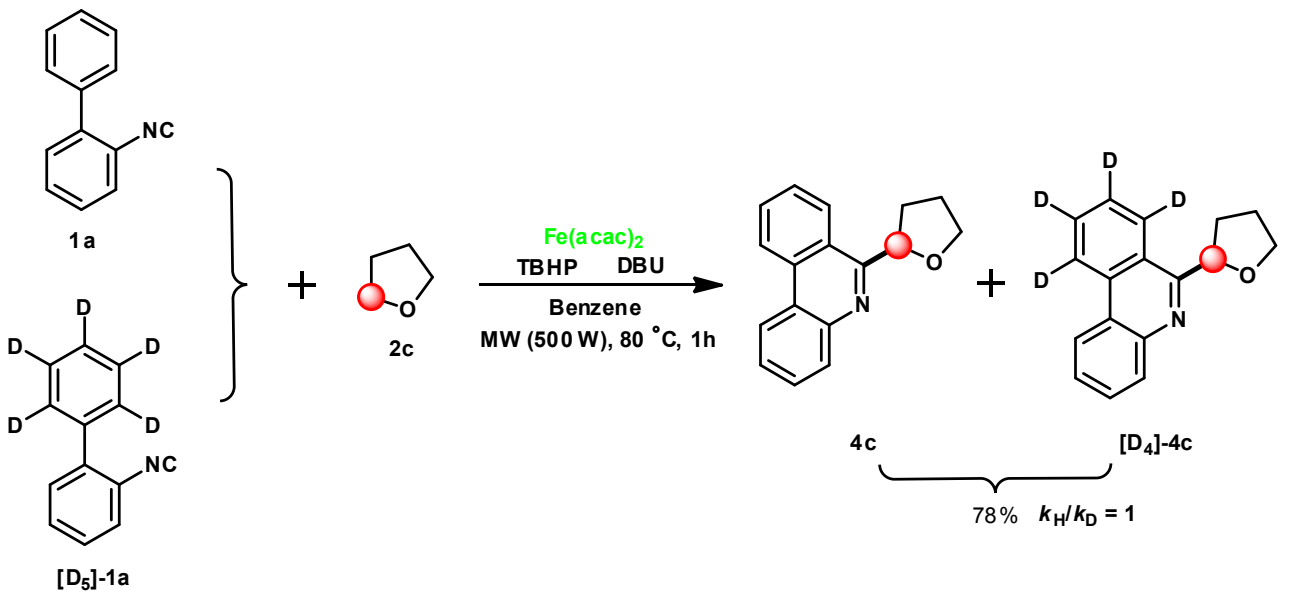

$k_{\mathrm{H}} / k_{\mathrm{D}}=0.5 / 0.5=1 / 1$. (The KIE was determined by ${ }^{1} \mathrm{H}$ NMR spectroscopy by analyzing the ratio of $4 c$ and $\left.\left[D_{4}\right]-4 c\right)$.

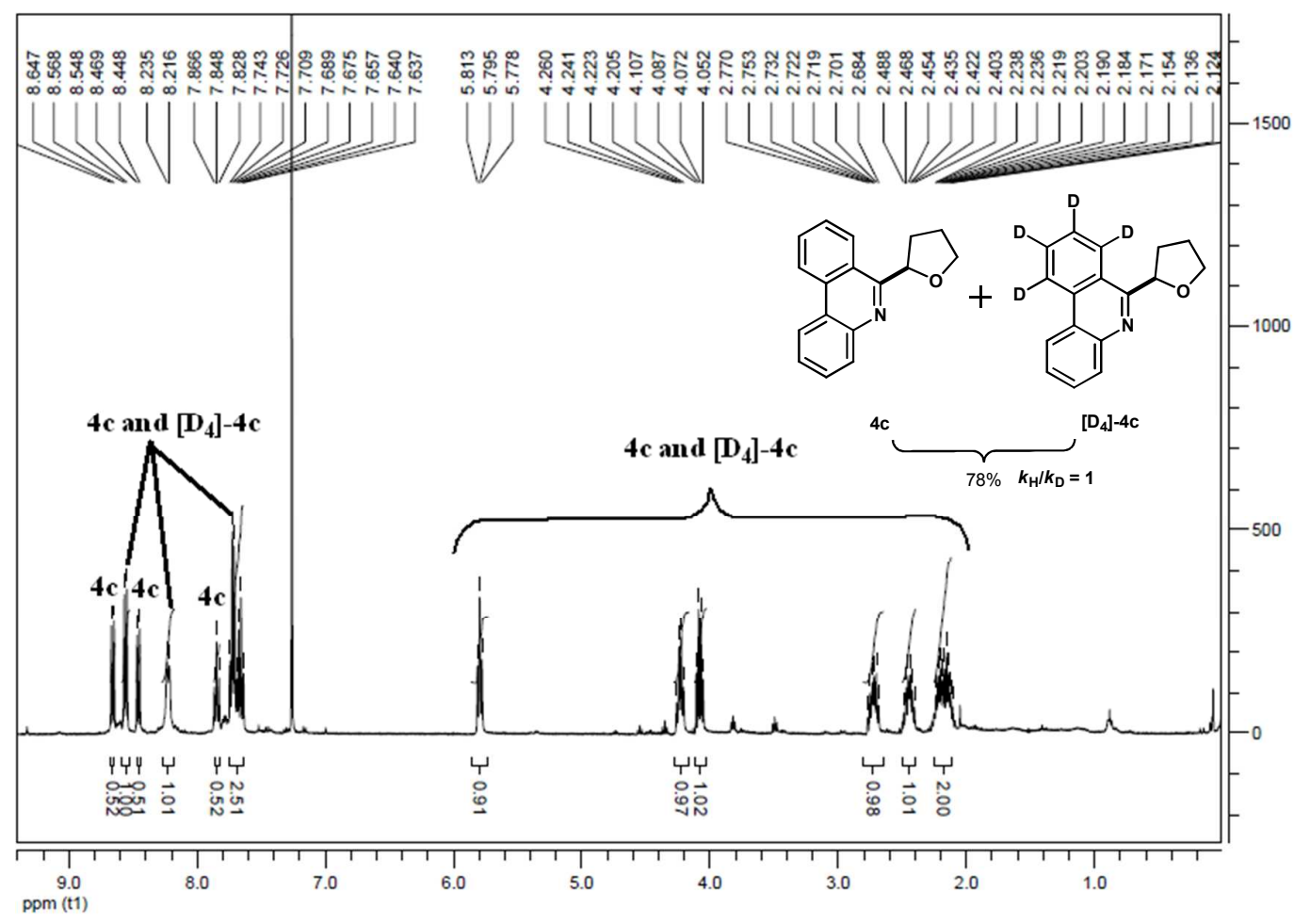


Intramolecular Kinetic Isotope Effect Study<smiles>[2H]c1ccccc1-c1ccccc1N=O</smiles>

[D, 1 -1a
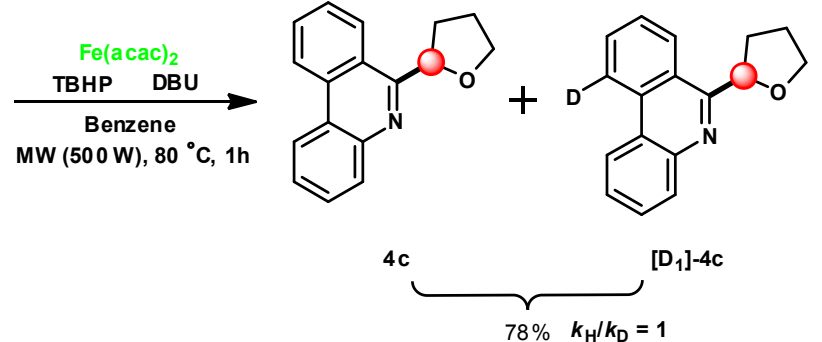

$k_{\mathrm{H}} / k_{\mathrm{D}}=0.5 / 0.5=1 / 1$. (The KIE was determined by ${ }^{1} \mathrm{H}$ NMR spectroscopy by analyzing the ratio of $4 c$ and $\left.\left[D_{1}\right]-4 c\right)$.

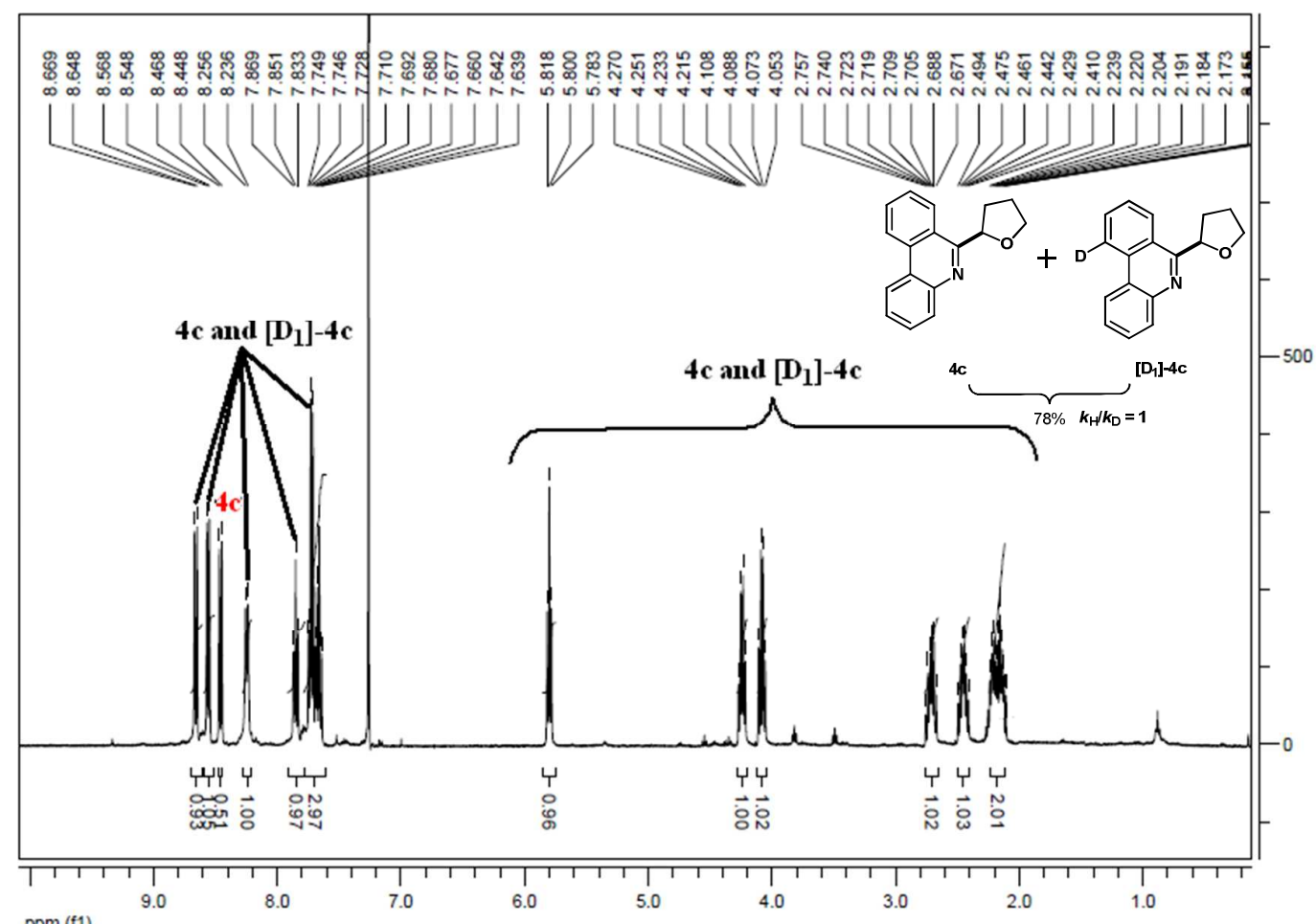

ppm (f1) 
III. Radical Inhibition Studies

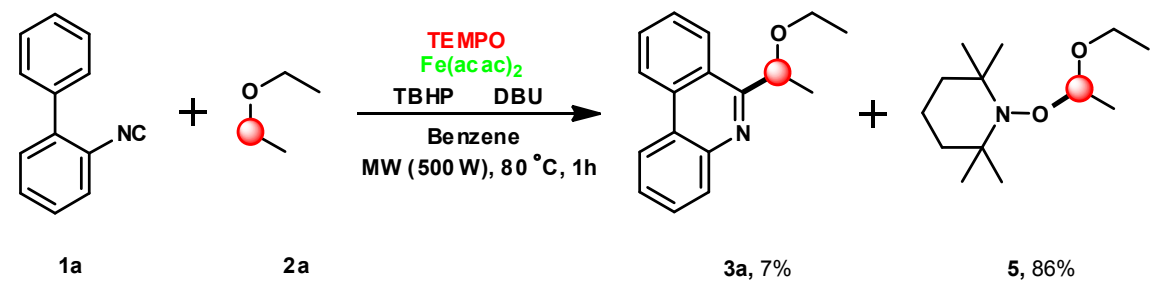

1-(1-ethoxyethoxy)-2,2,6,6-tetramethylpiperidine (5)

Proton NMR $\left(\mathrm{CDCl}_{3}\right)$

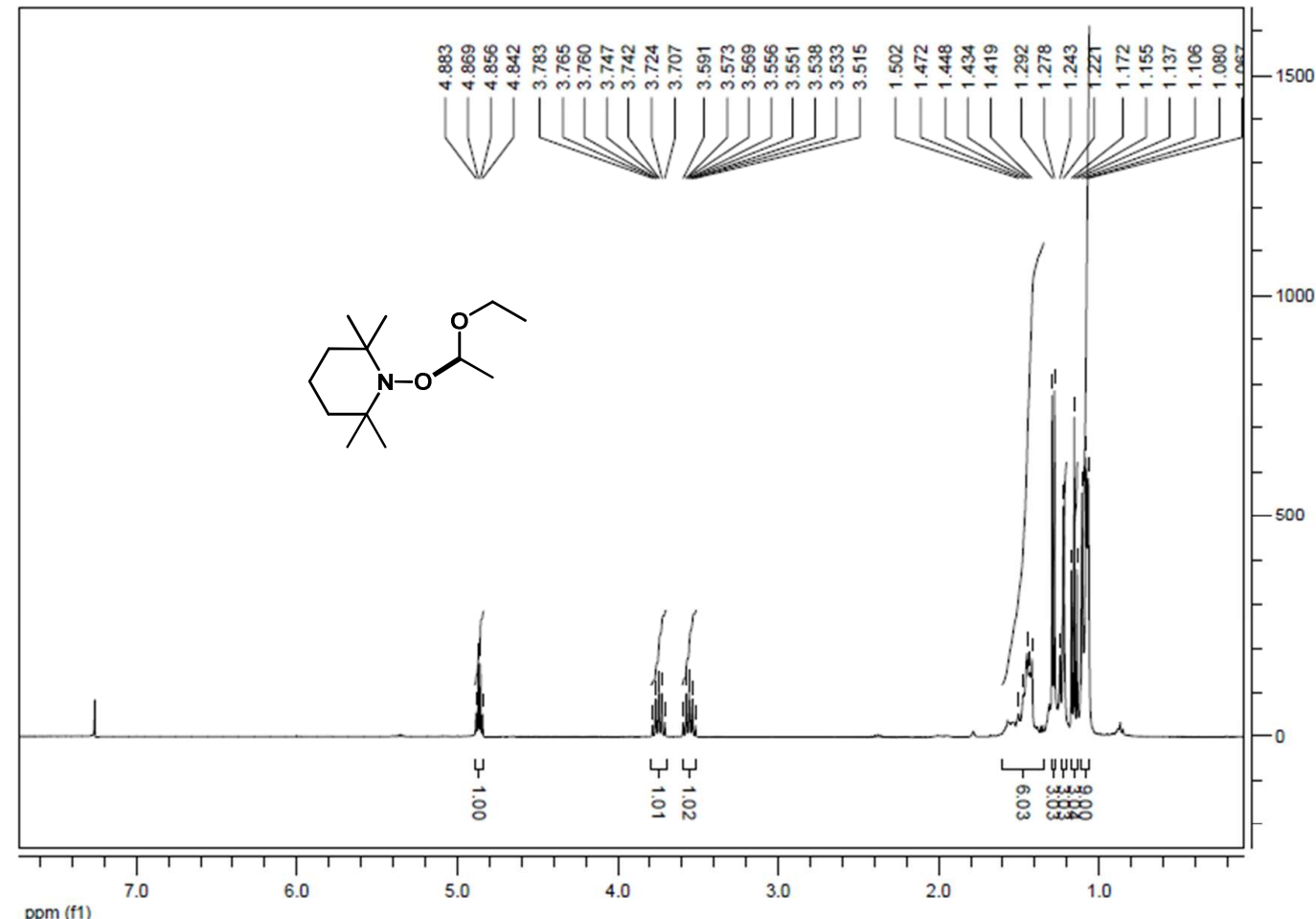

ppm (f1) 


\section{Carbon NMR $\left(\mathrm{CDCl}_{3}\right)$}

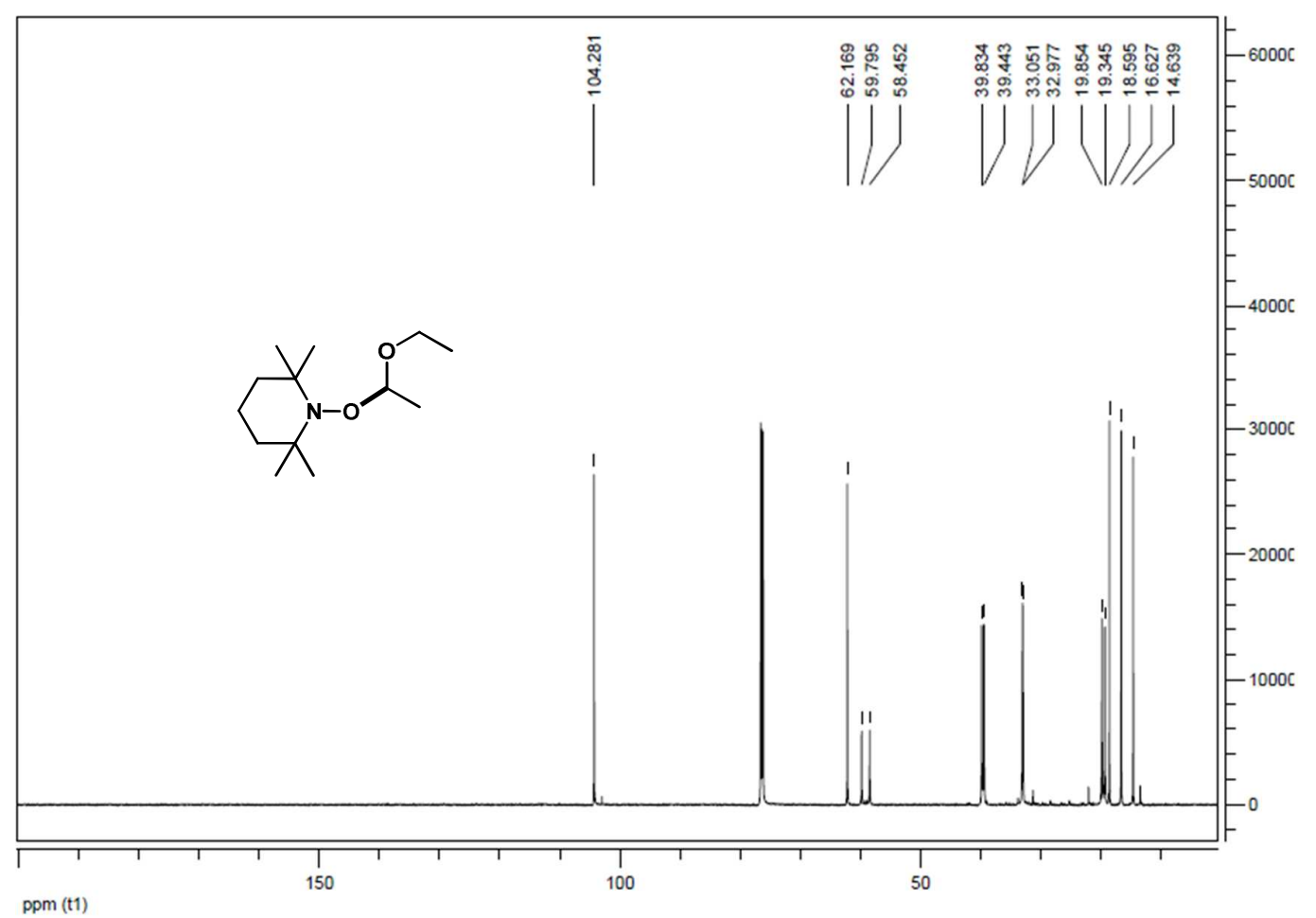


IV. ${ }^{1} \mathrm{H}$ and ${ }^{13} \mathrm{C}$ NMR

2-isocyano-1,1'-biphenyl (1a)

Proton NMR $\left(\mathrm{CDCl}_{3}\right)$

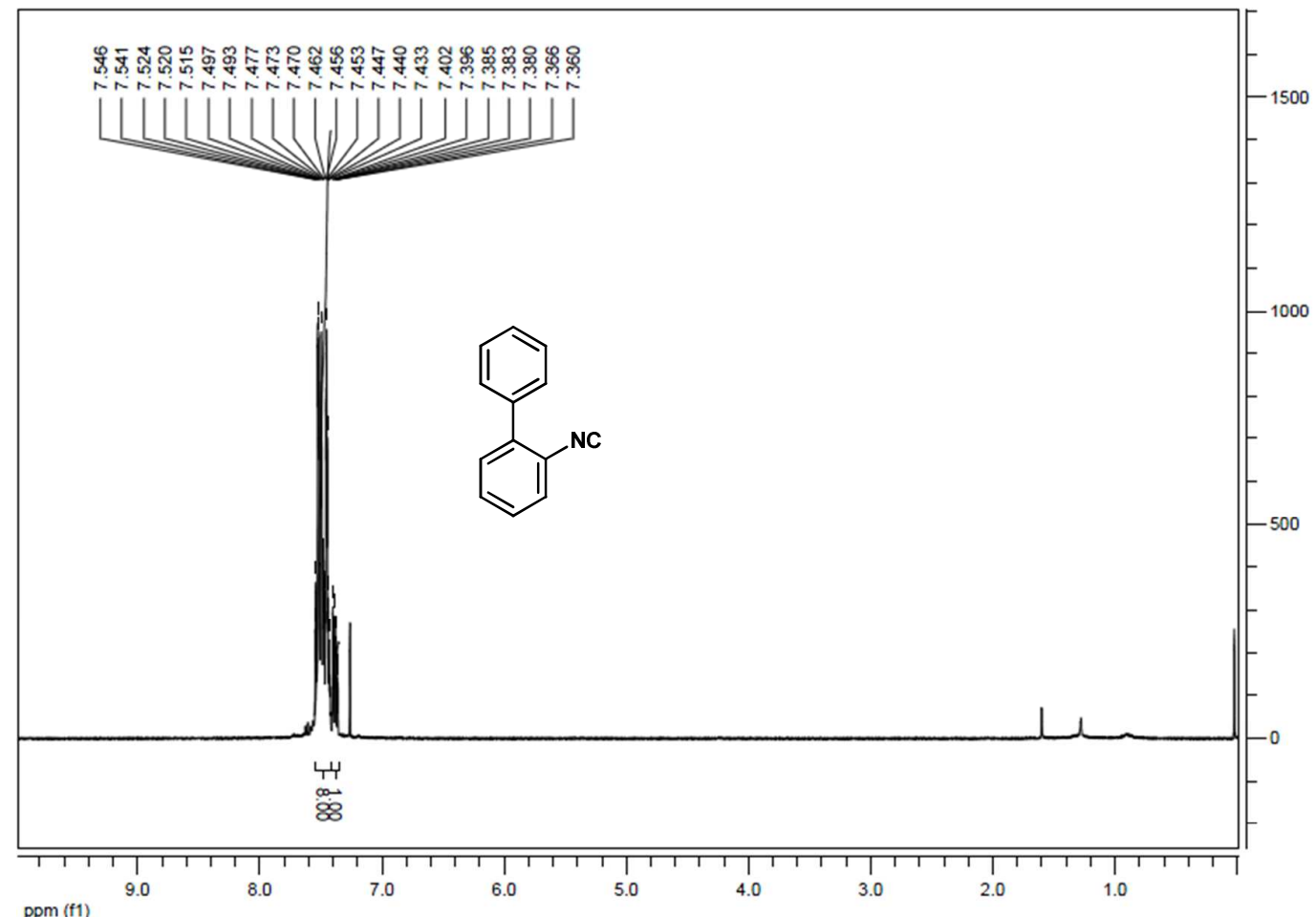

Carbon NMR (CDCl $\left.)_{3}\right)$

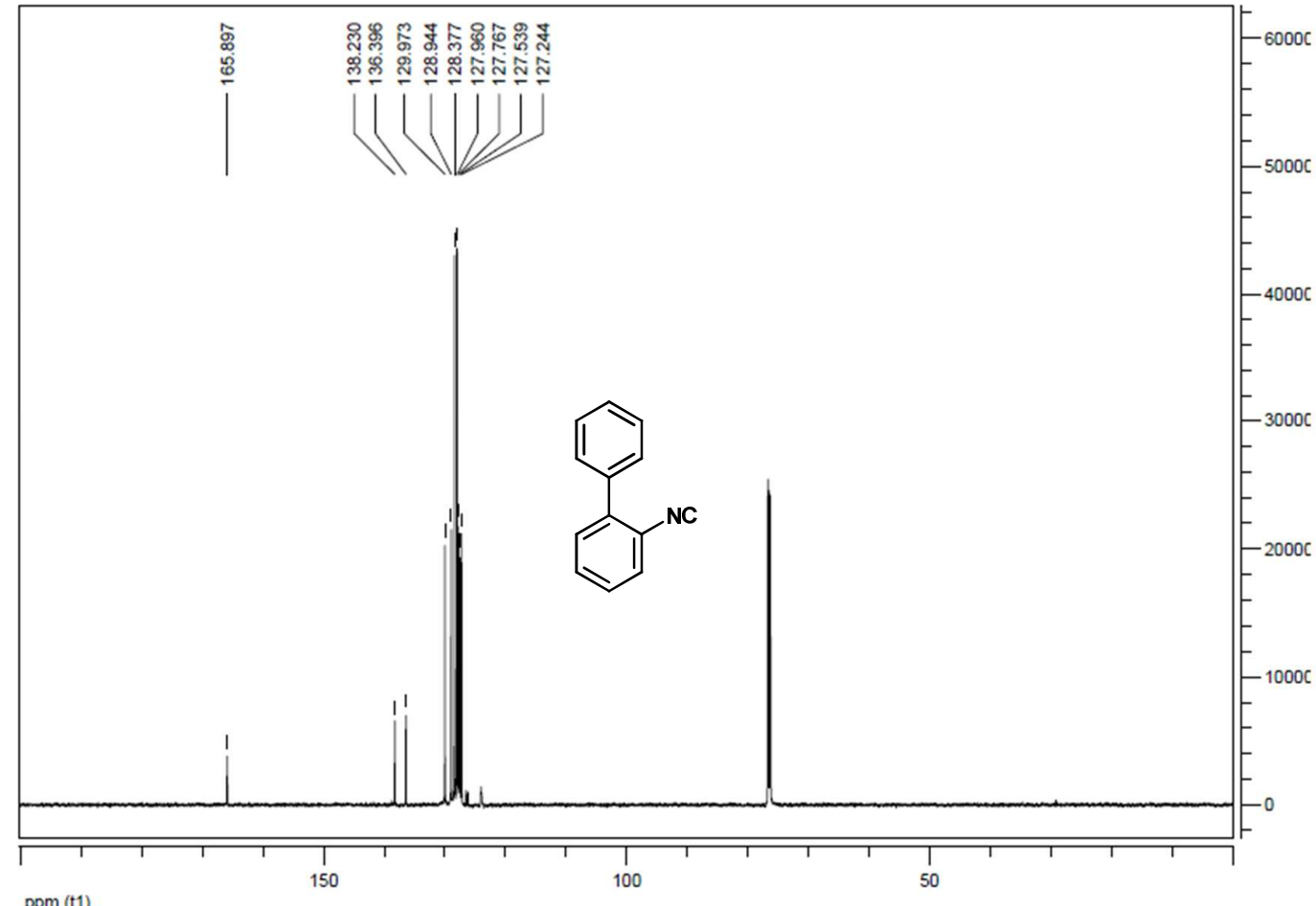

ppm (t1) 
2-isocyano-5-methyl-1,1'-biphenyl (1b)

Proton NMR $\left(\mathrm{CDCl}_{3}\right)$

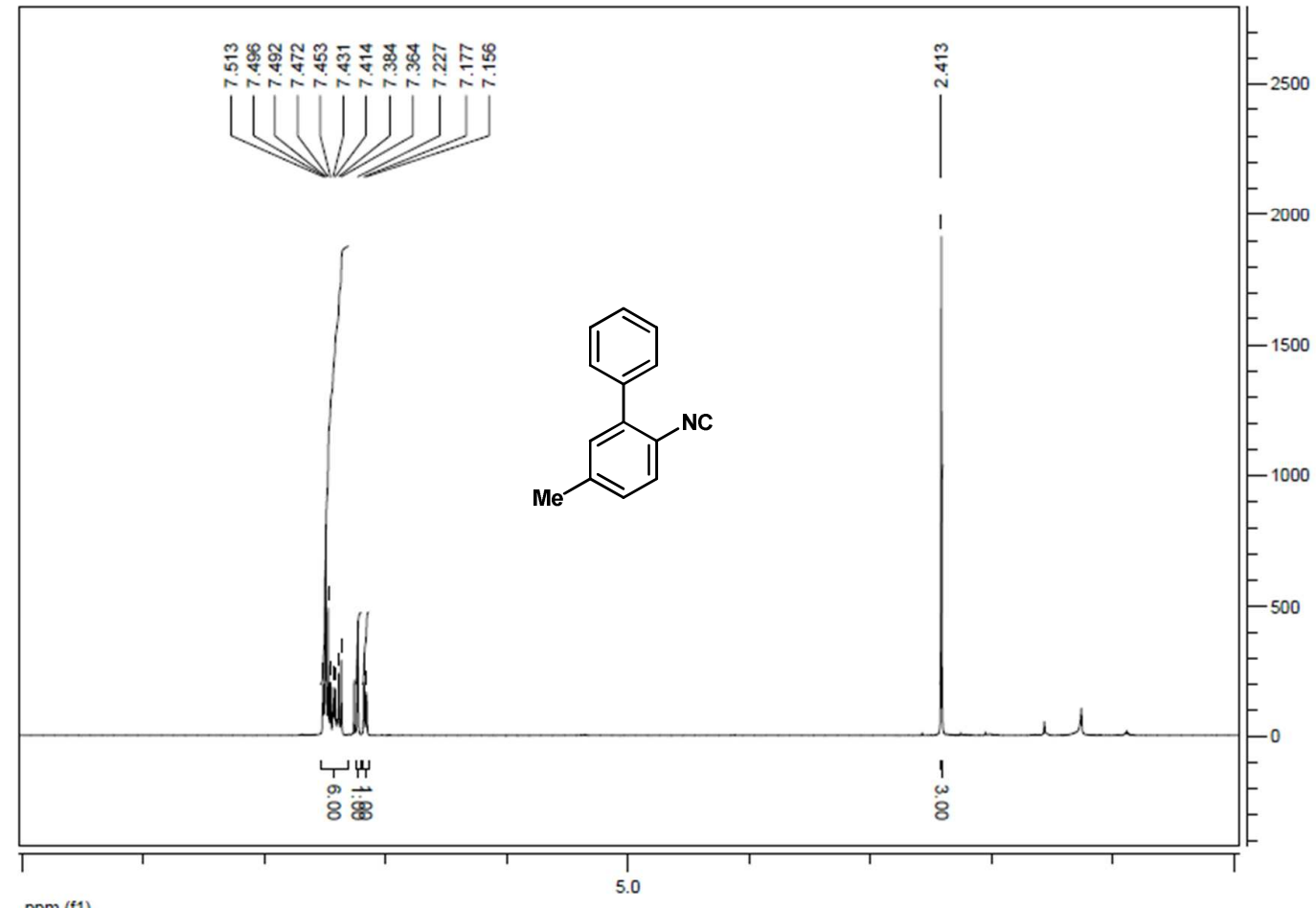

Carbon NMR (CDCl $\left.)_{3}\right)$

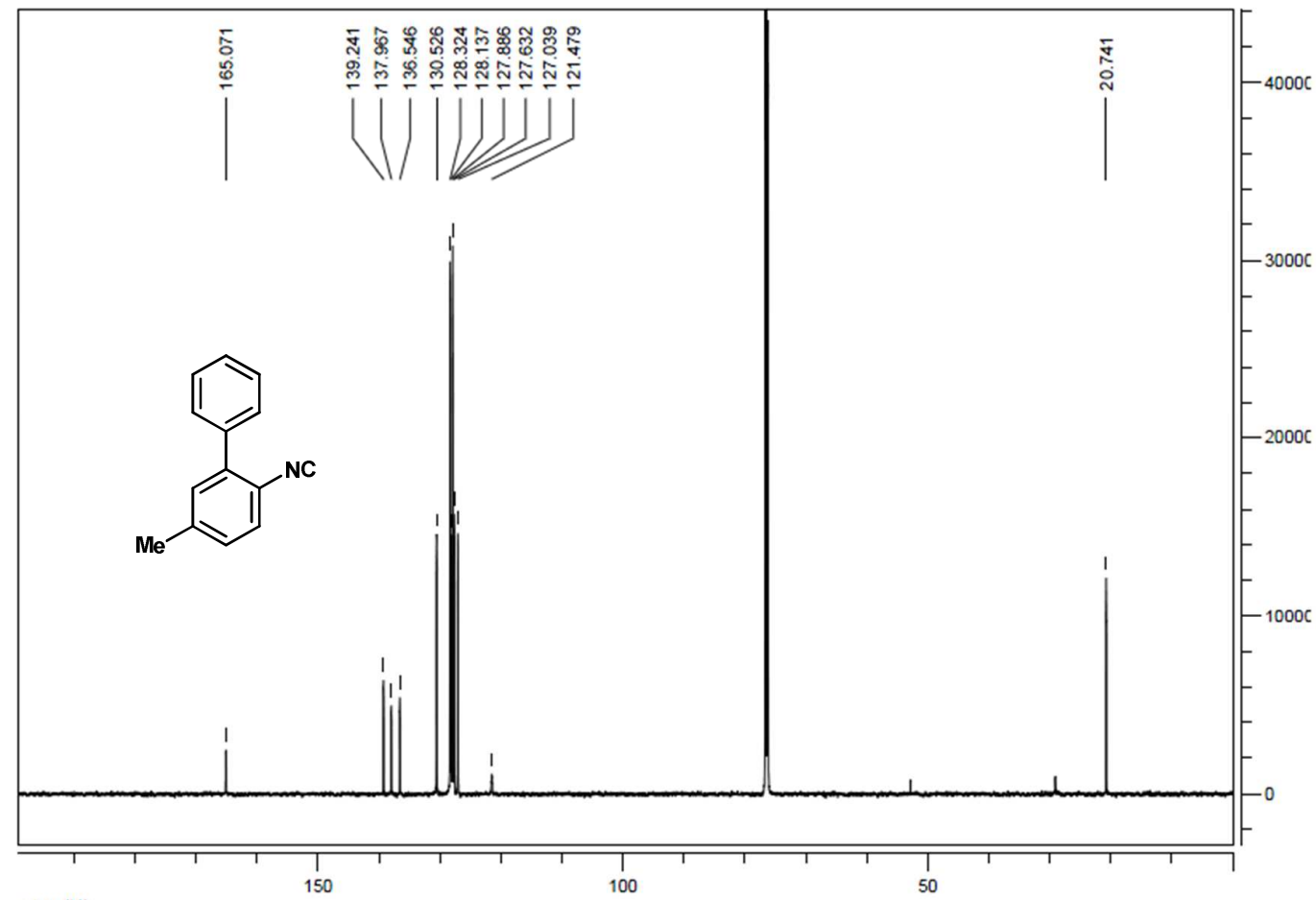

$\mathrm{ppm}(\mathrm{t} 1)$ 
2-isocyano-5-methoxy-1,1'-biphenyl (1c)

Proton NMR $\left(\mathrm{CDCl}_{3}\right)$

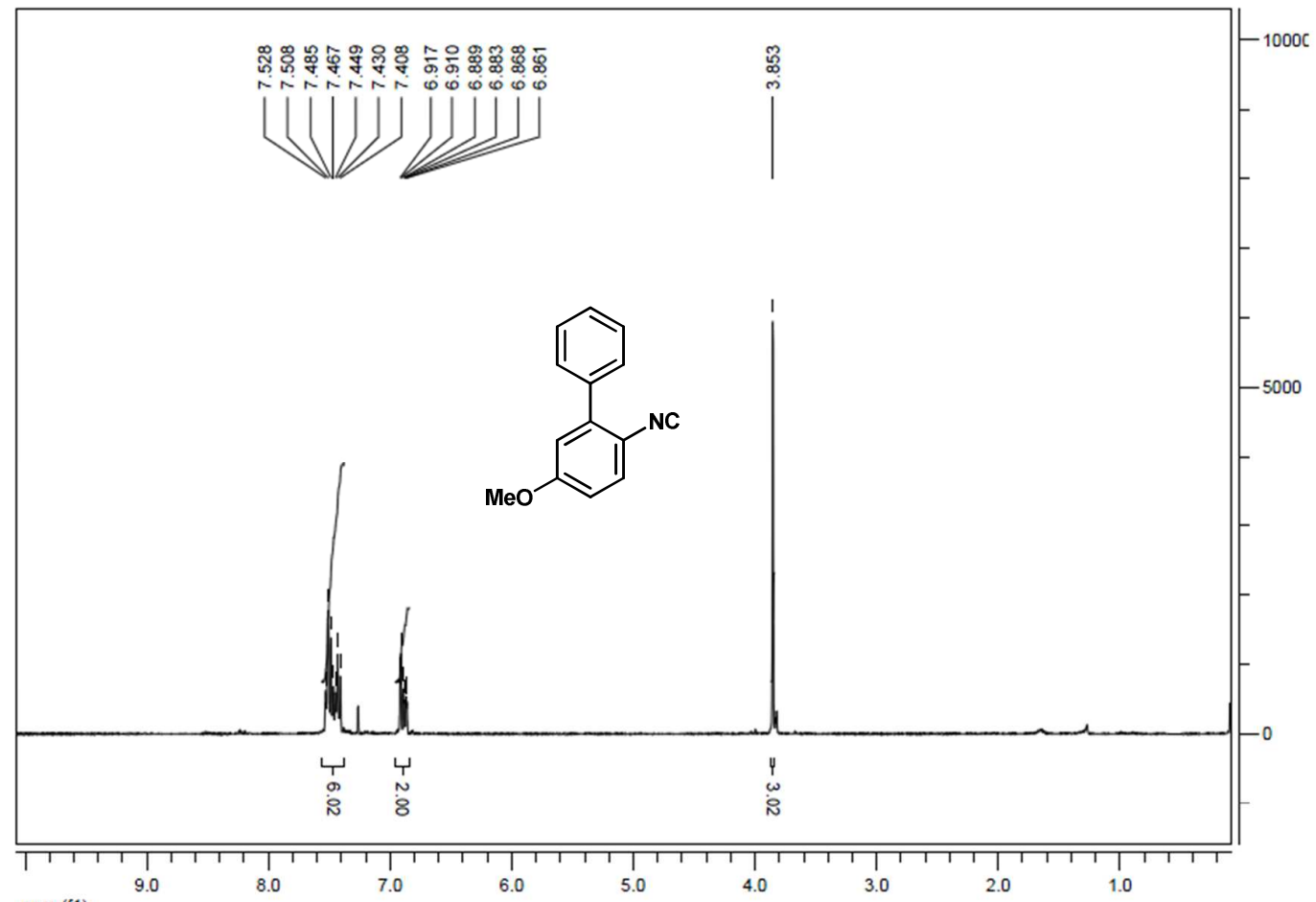

ppm (f1)

\section{Carbon NMR (CDCl $)_{3}$}

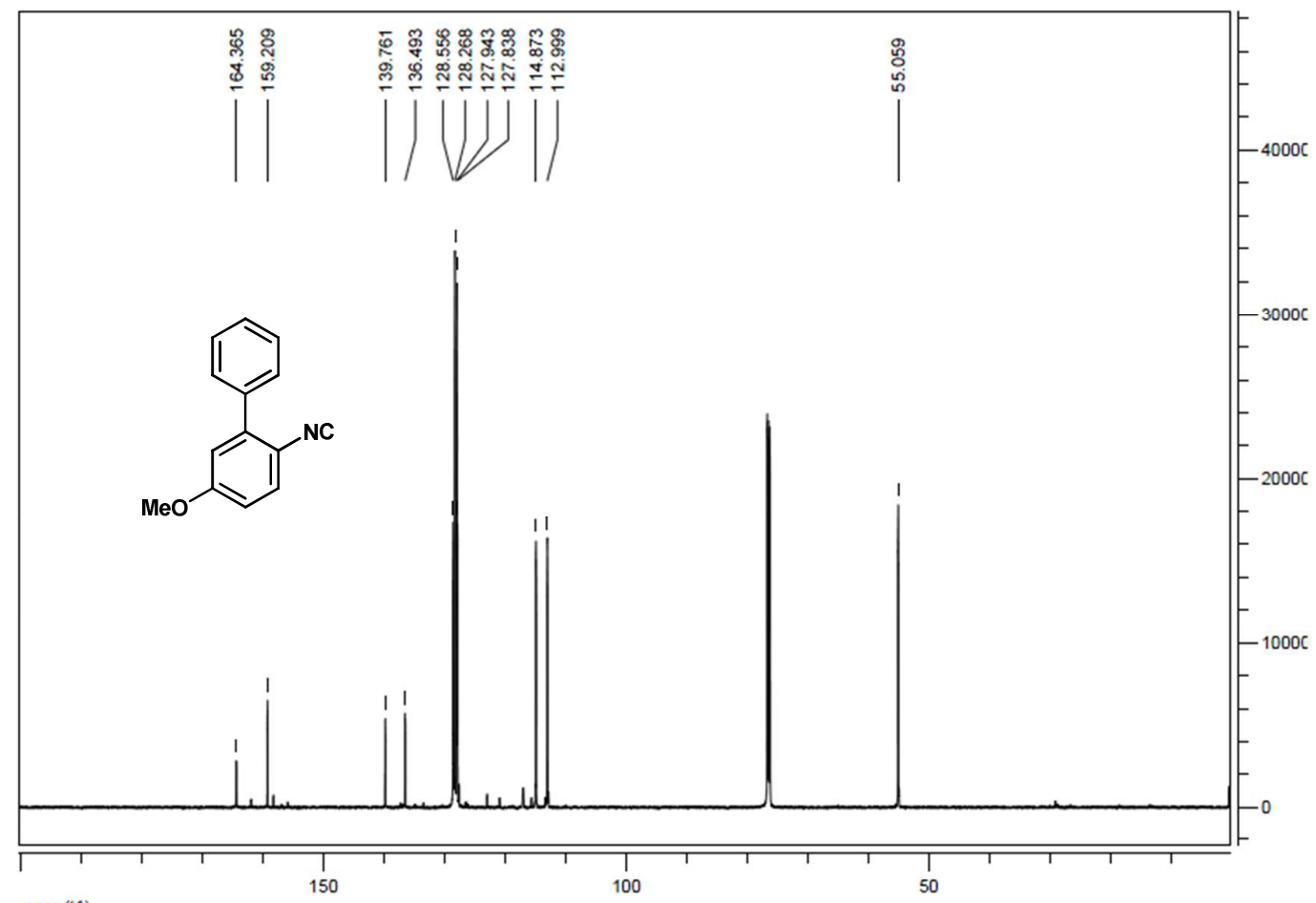

ppm (t1) 
5-fluoro-2-isocyano-1,1'-biphenyl (1d)

Proton NMR $\left(\mathrm{CDCl}_{3}\right)$

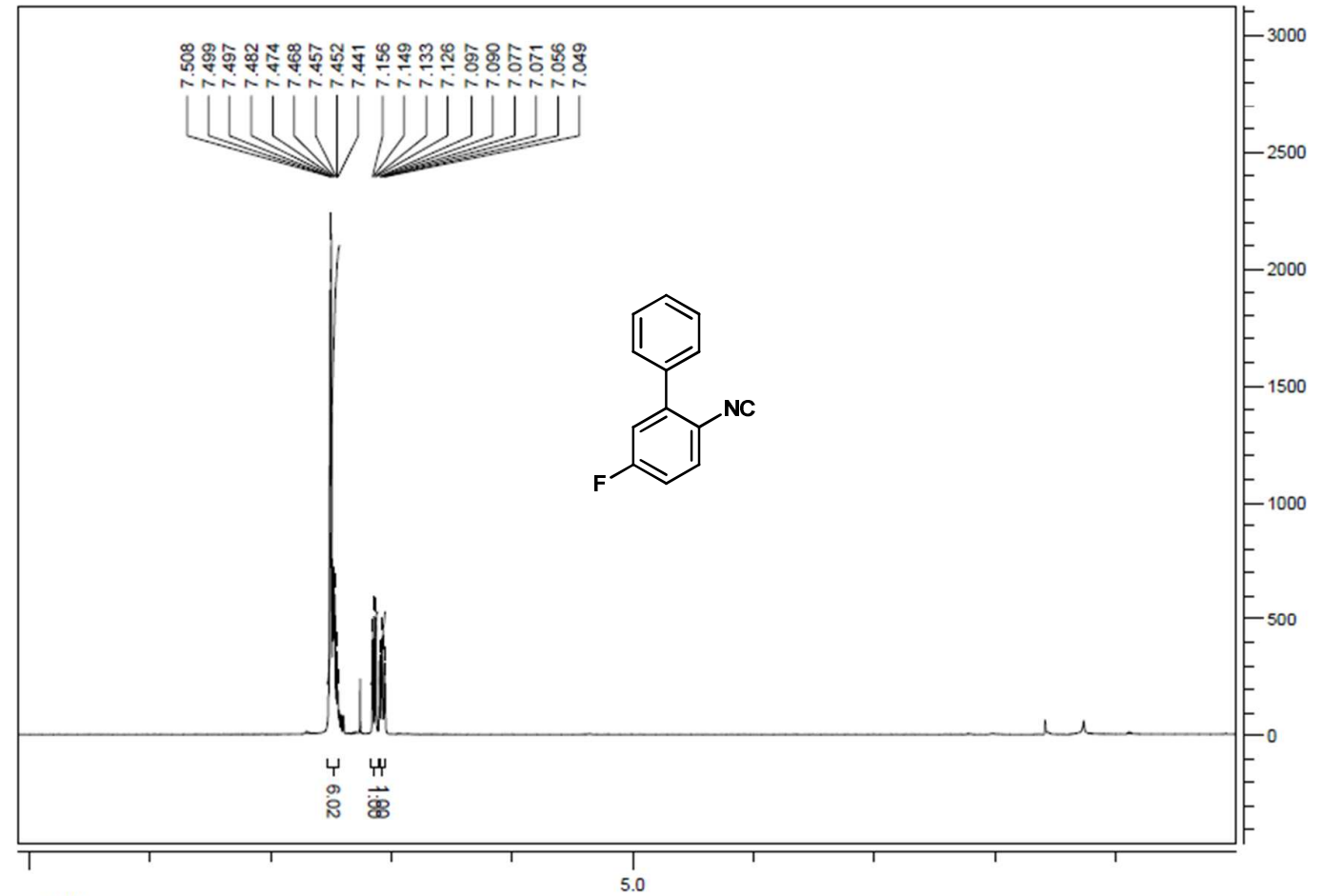

ppm (11)

\section{Carbon NMR (CDCl $)_{3}$}

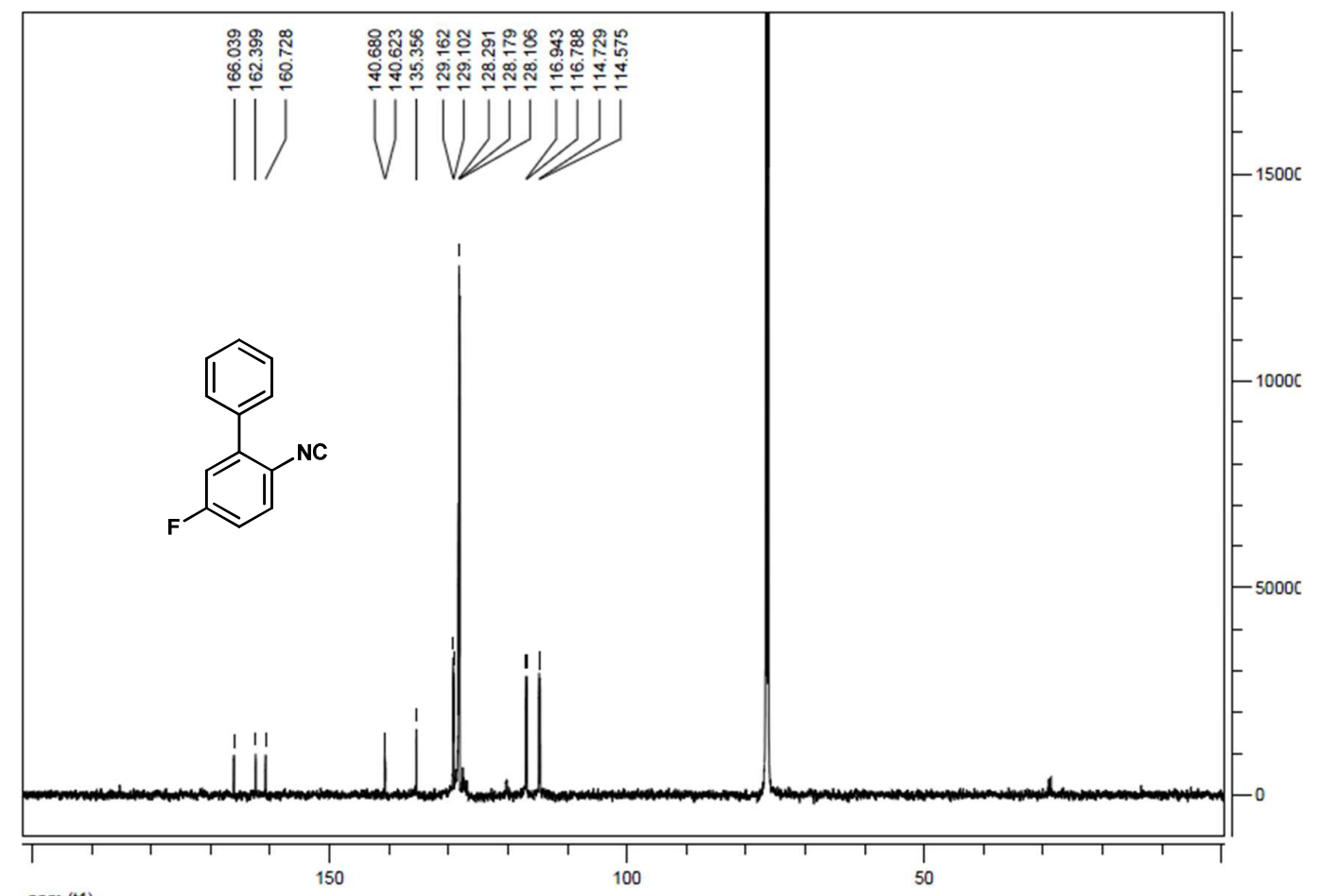

ppm (t1) 
5-chloro-2-isocyano-1,1'-biphenyl (1e)

Proton NMR $\left(\mathrm{CDCl}_{3}\right)$

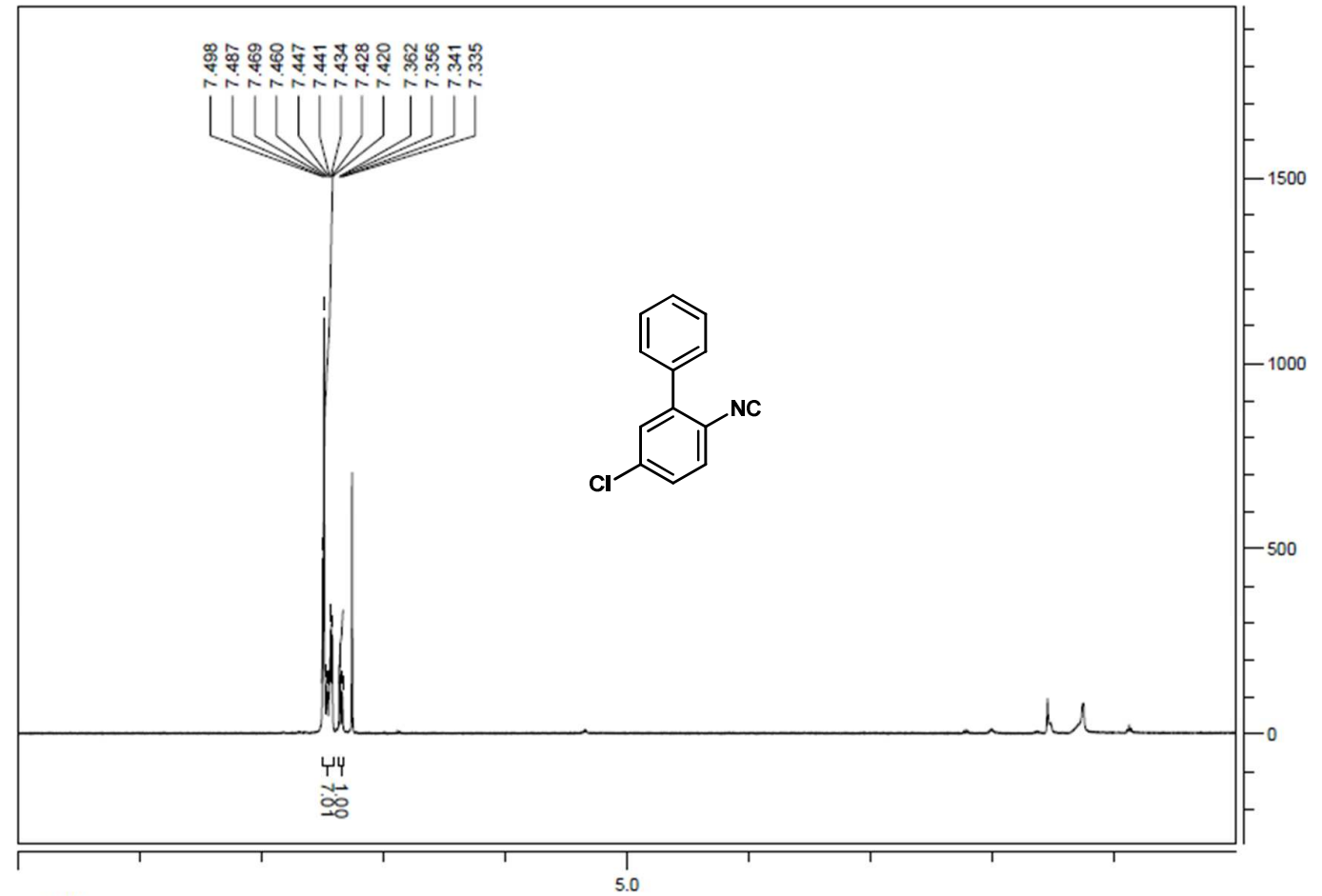

ppm (f1)

\section{Carbon NMR (CDCl $)_{3}$}

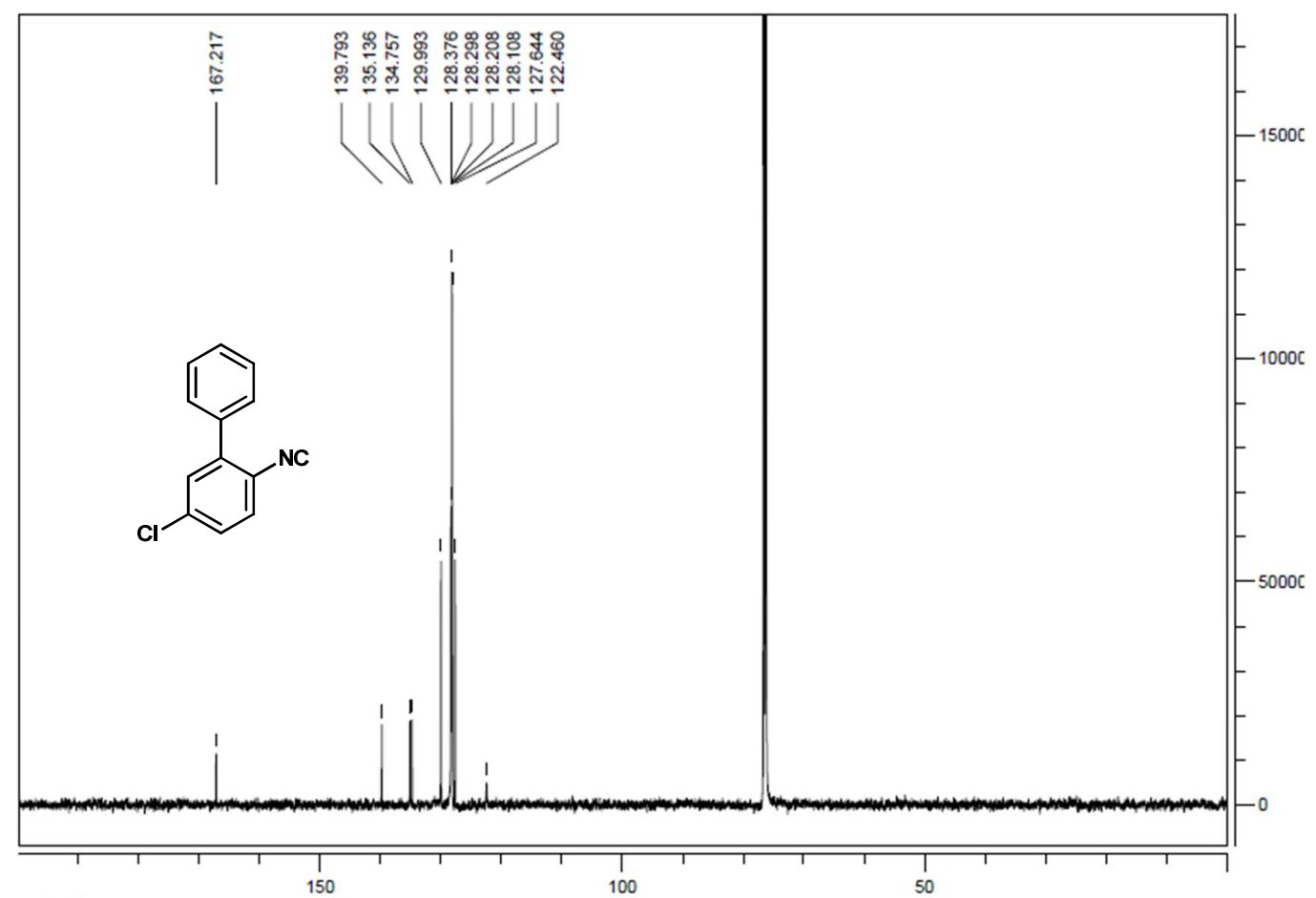

$\mathrm{ppm}(\mathrm{t} 1)$ 
2-isocyano-5-(trifluoromethyl)-1,1'-biphenyl (1f)

Proton NMR $\left(\mathrm{CDCl}_{3}\right)$

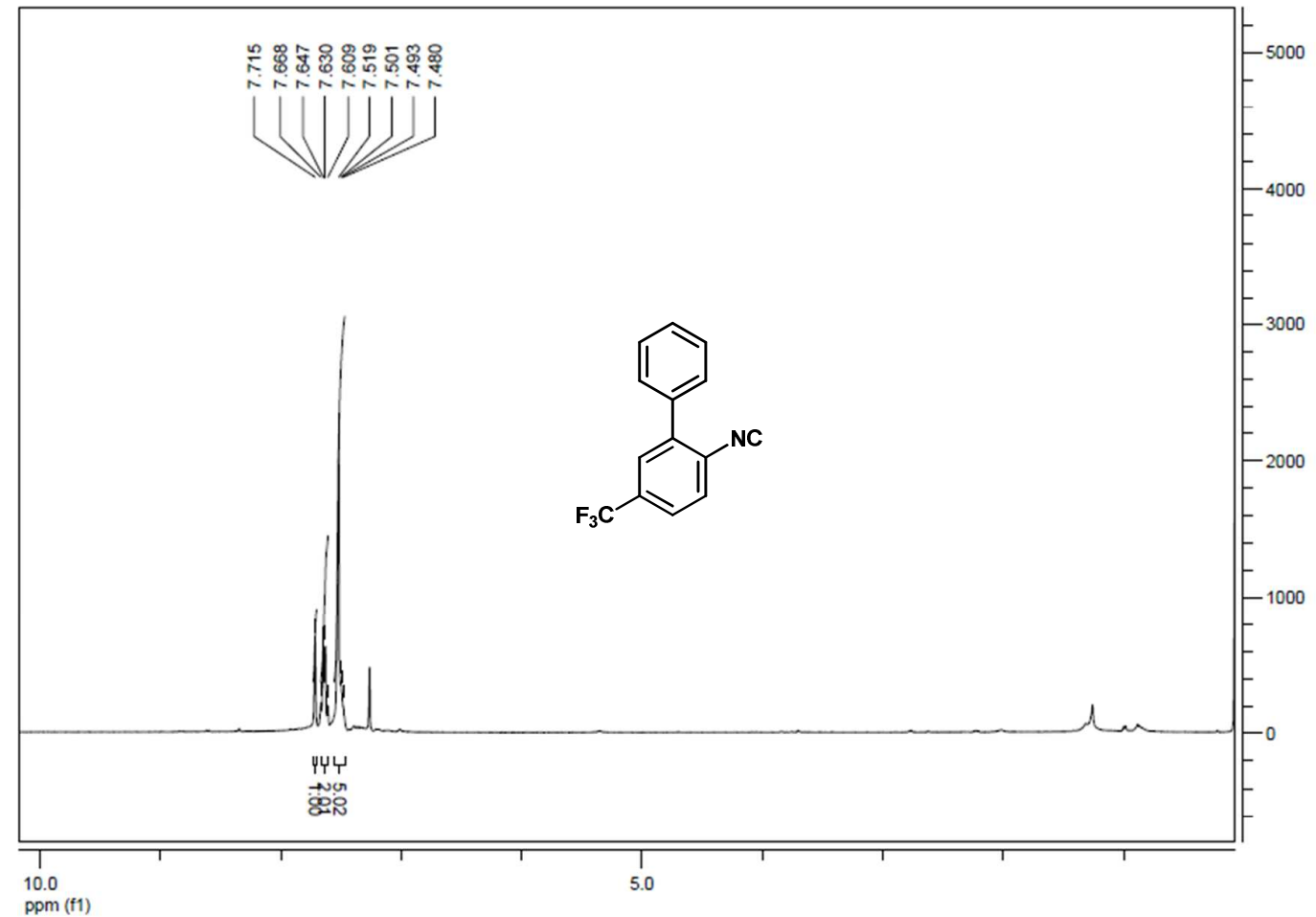

Carbon NMR (CDCl $\left.)_{3}\right)$

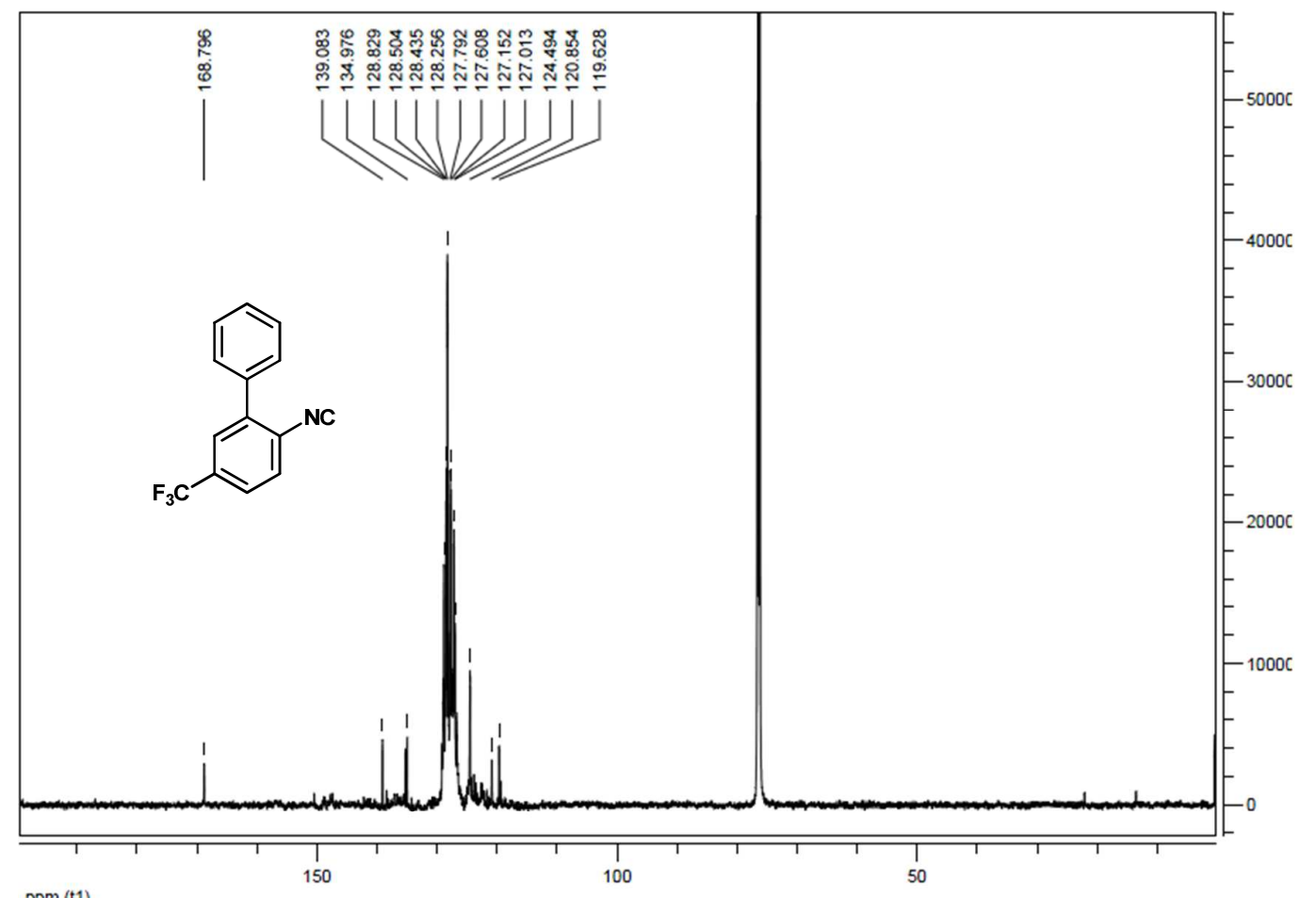

ppm (t1) 
2-isocyano-4'-methyl-1,1'-biphenyl (1g)

Proton NMR $\left(\mathrm{CDCl}_{3}\right)$

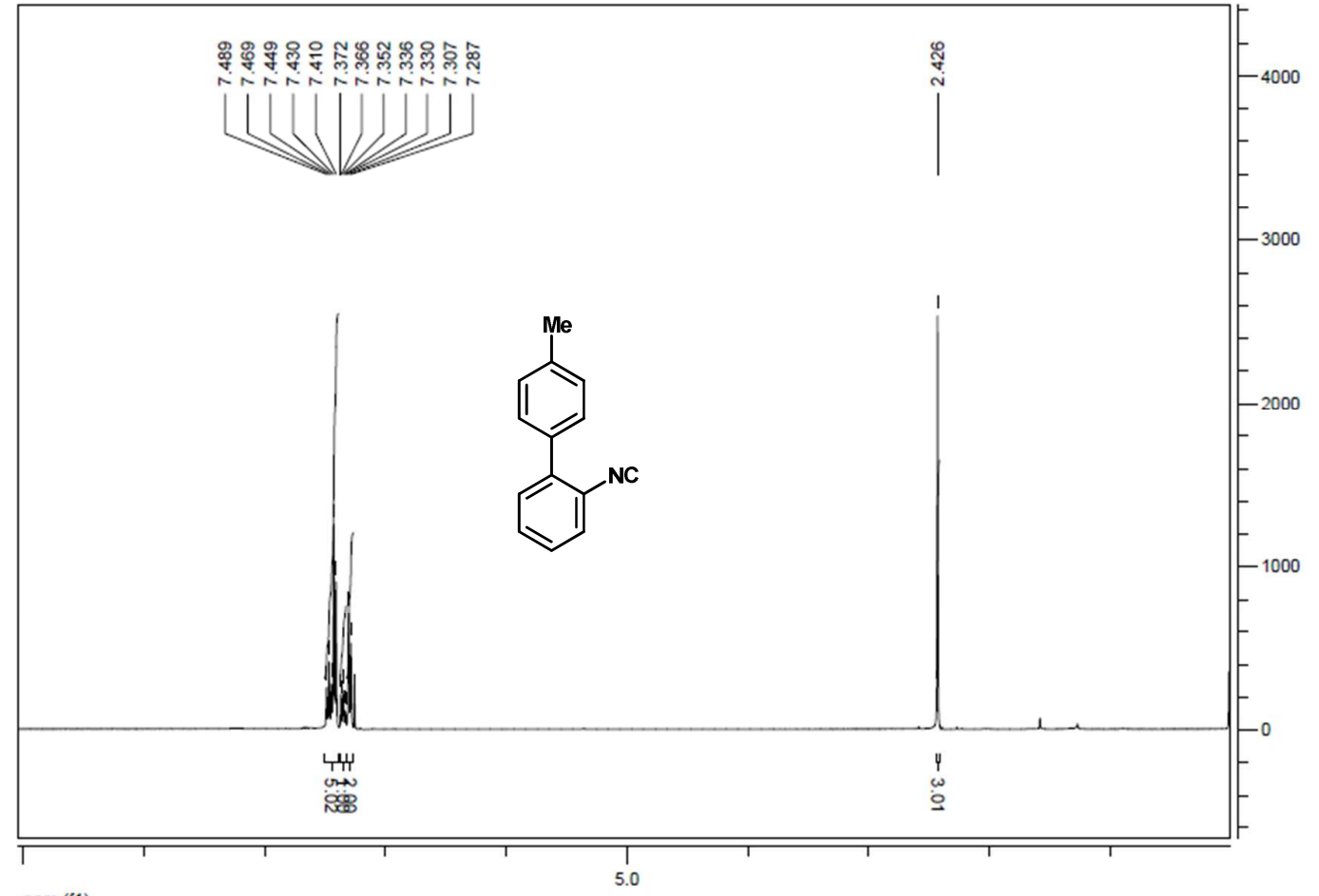

ppm (f1)

\section{Carbon NMR (CDCl $)_{3}$}

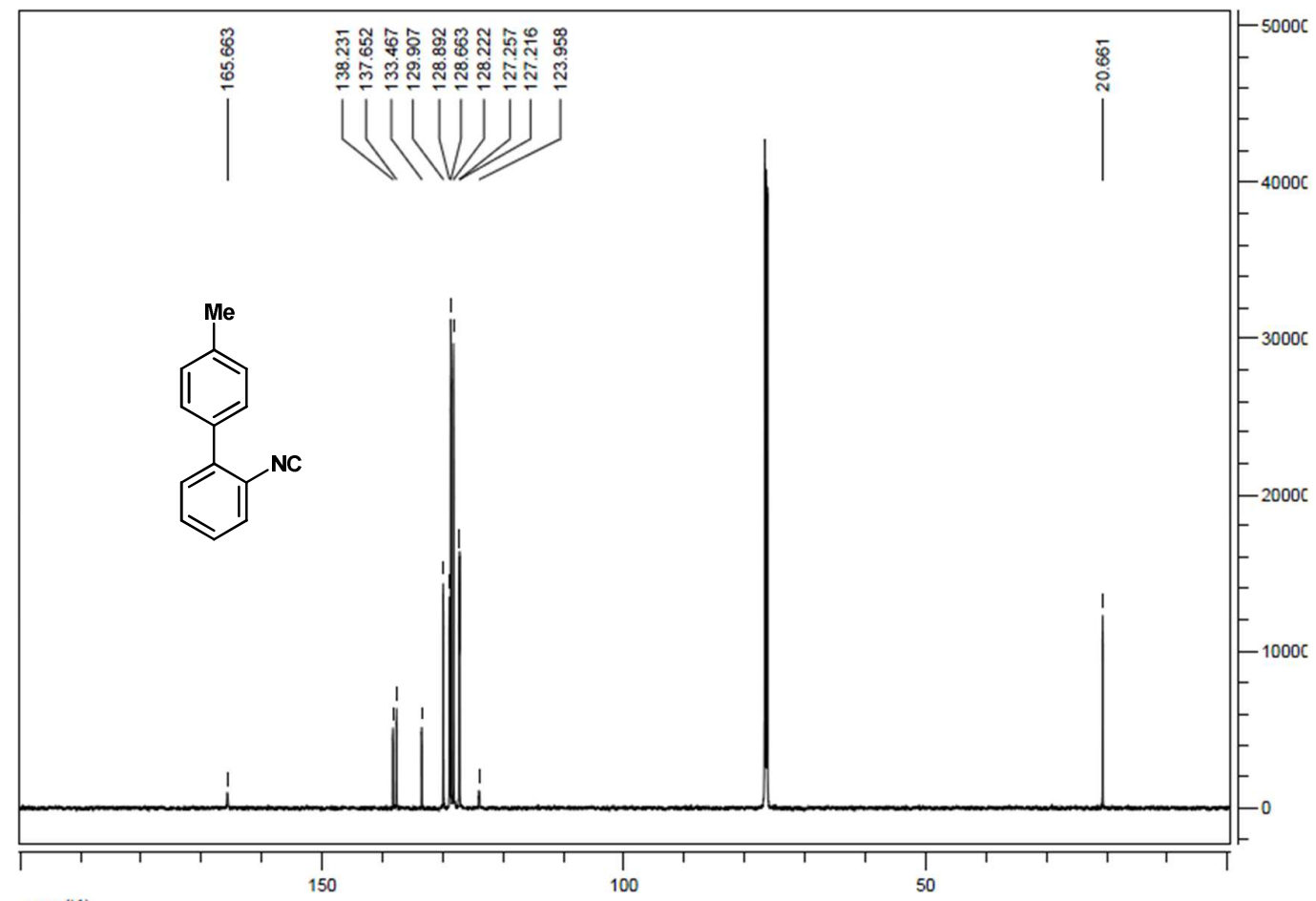

ppm (t1) 
2-isocyano-4'-methoxy-1,1'-biphenyl (1h)

Proton NMR $\left(\mathrm{CDCl}_{3}\right)$

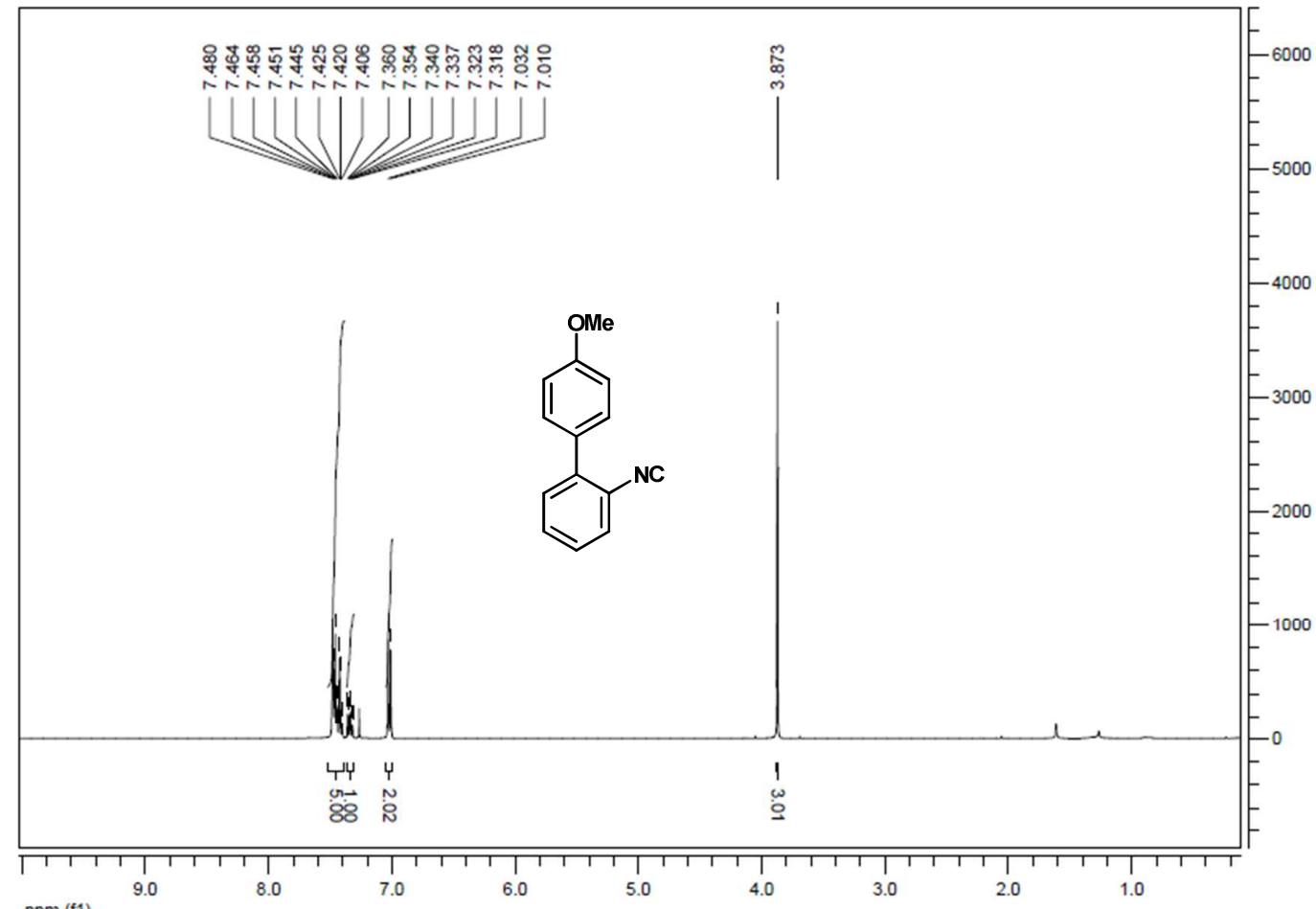

Carbon NMR $\left(\mathrm{CDCl}_{3}\right)$

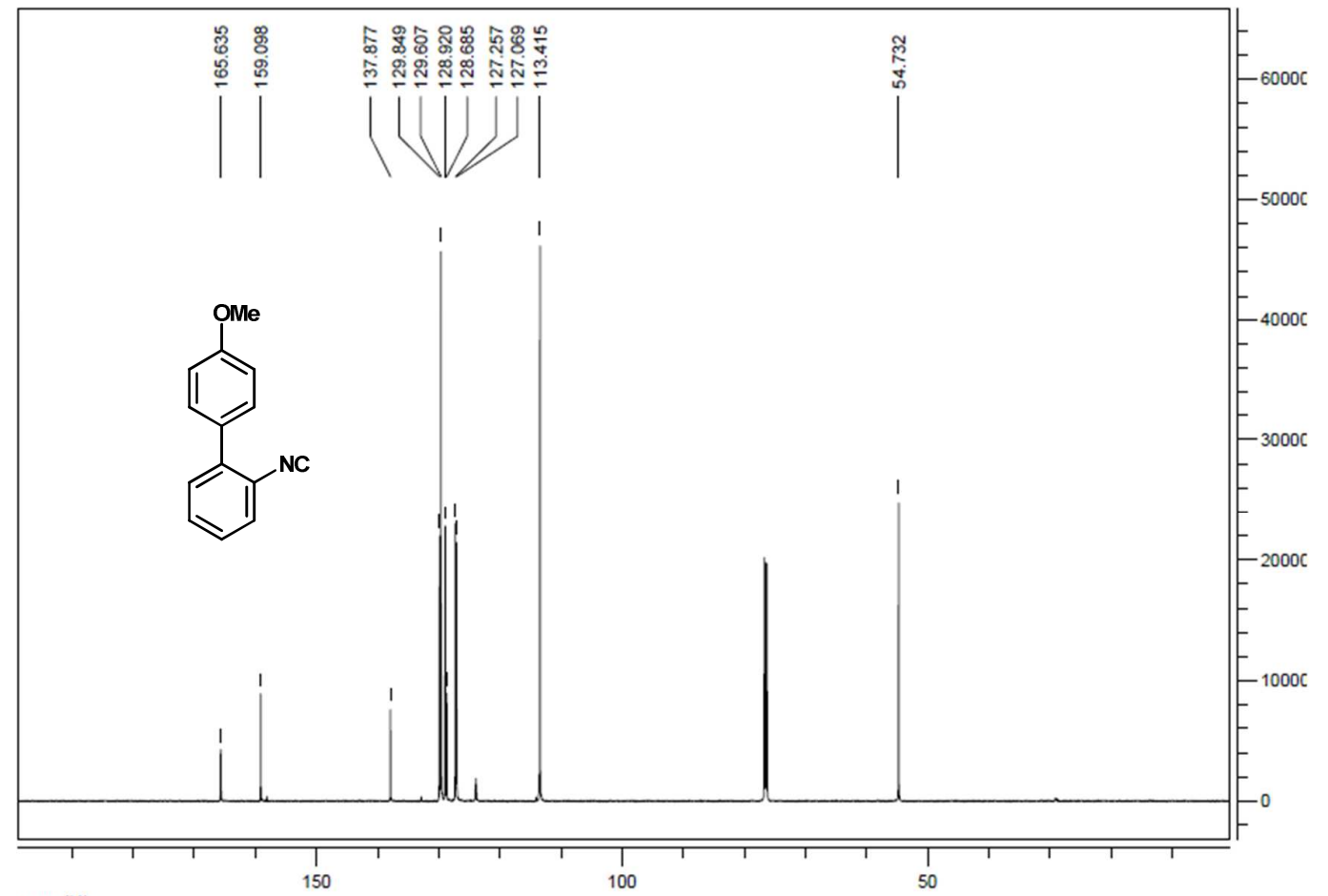

ppm (t1) 
4'-fluoro-2-isocyano-1,1'-biphenyl (1i)

Proton NMR $\left(\mathrm{CDCl}_{3}\right)$

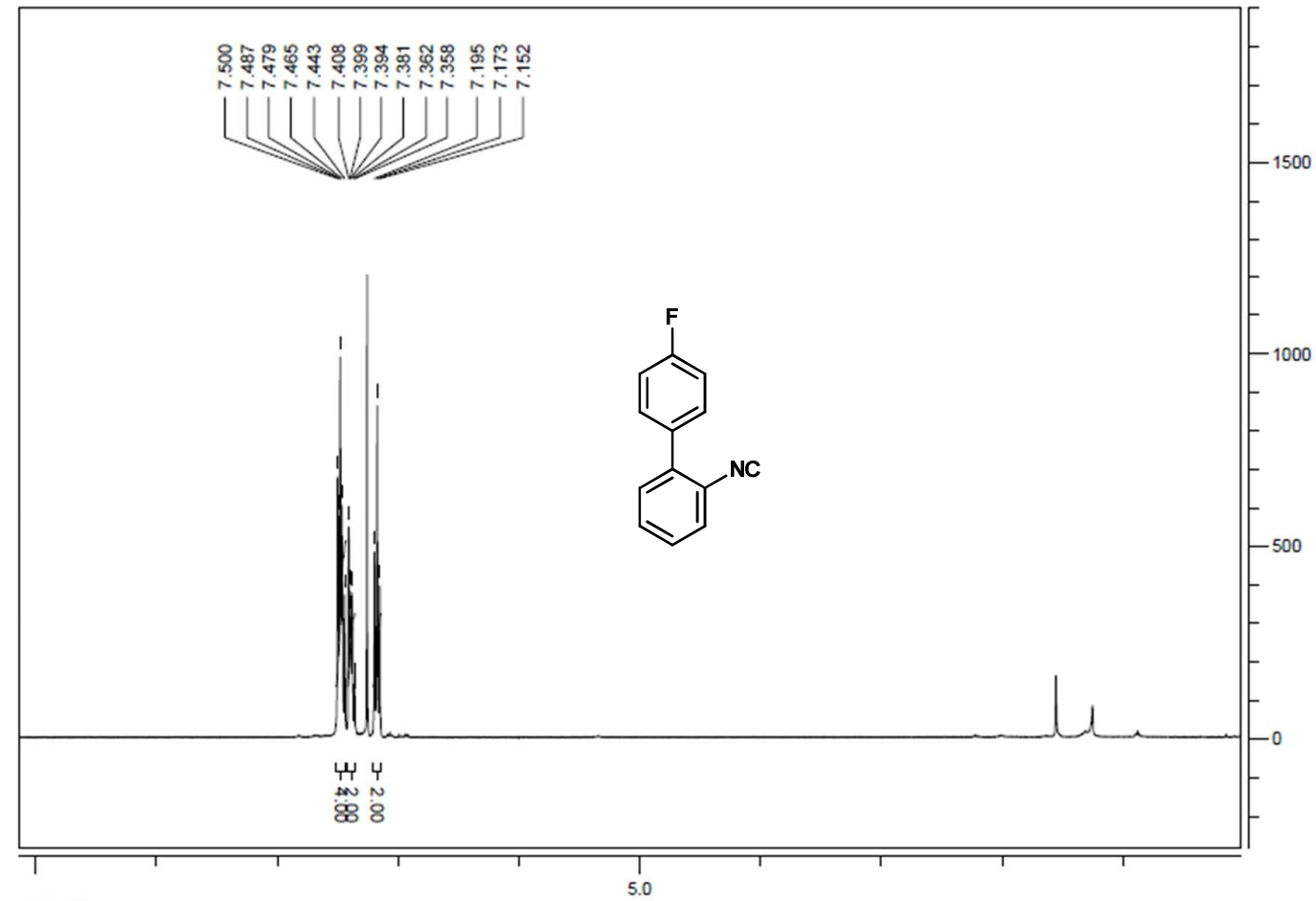

ppm (11)

\section{Carbon NMR (CDCl $)_{3}$}

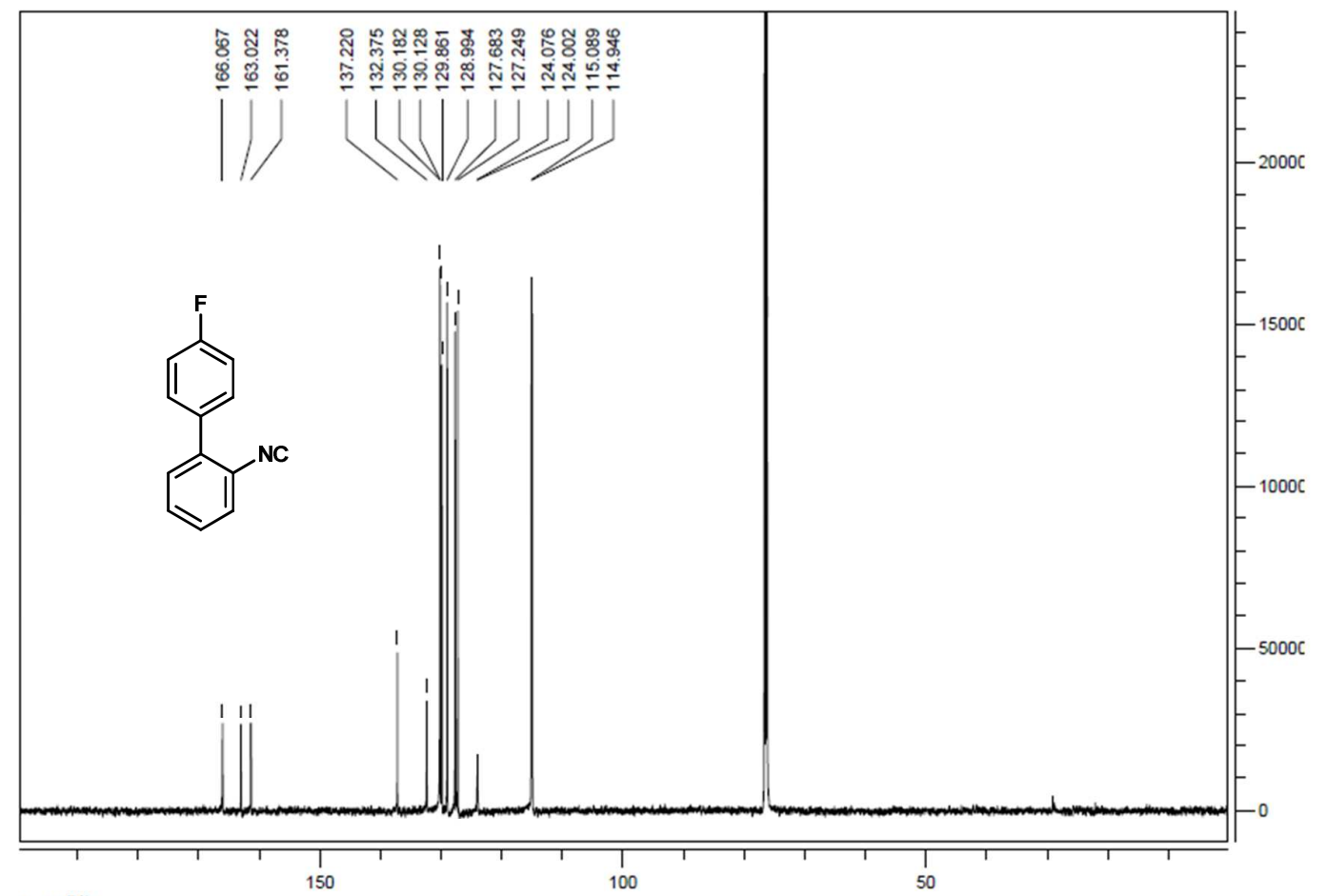

ppm (t1) 
4'-chloro-2-isocyano-1,1'-biphenyl (1j)

Proton NMR $\left(\mathrm{CDCl}_{3}\right)$

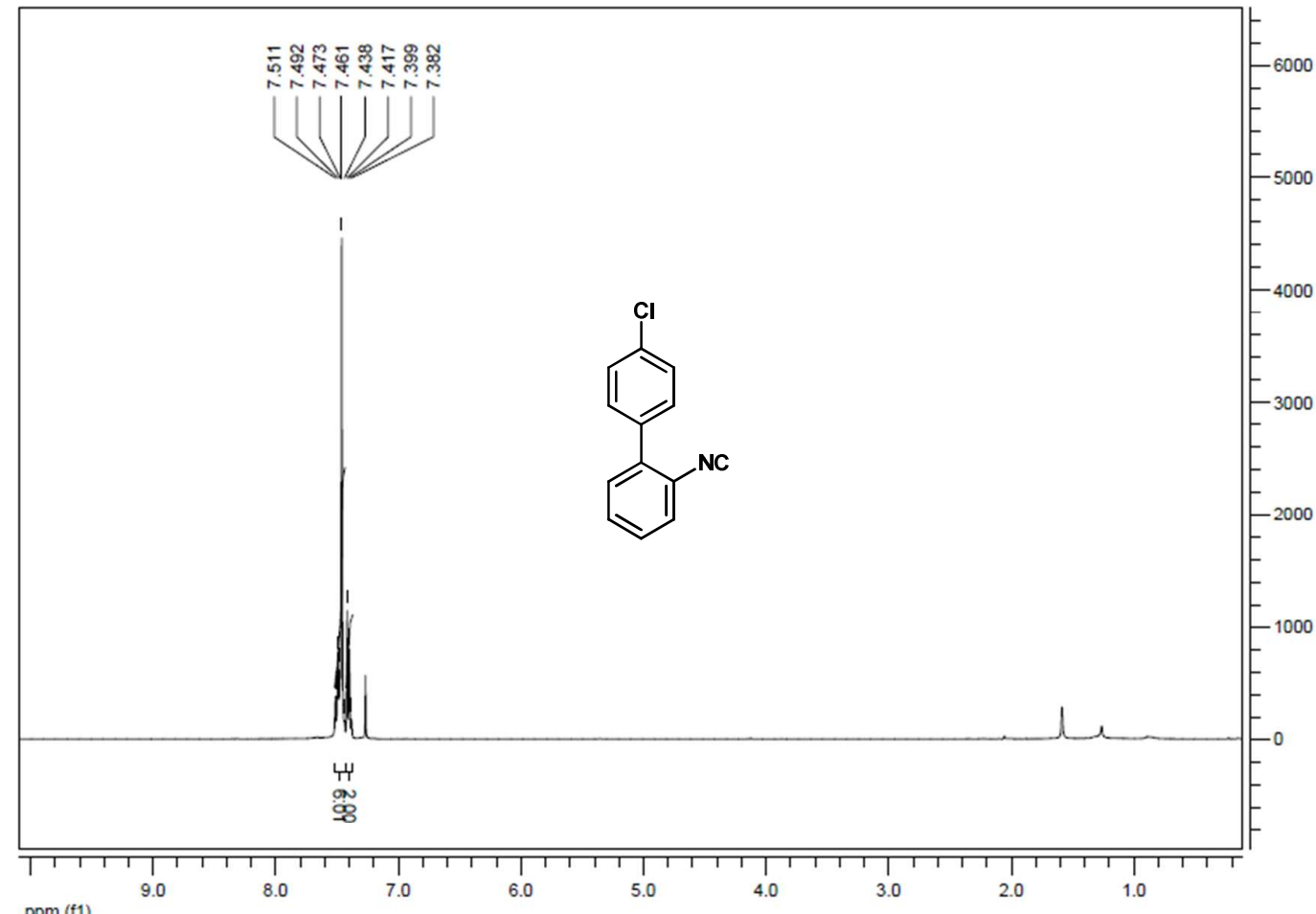

Carbon NMR $\left(\mathrm{CDCl}_{3}\right)$

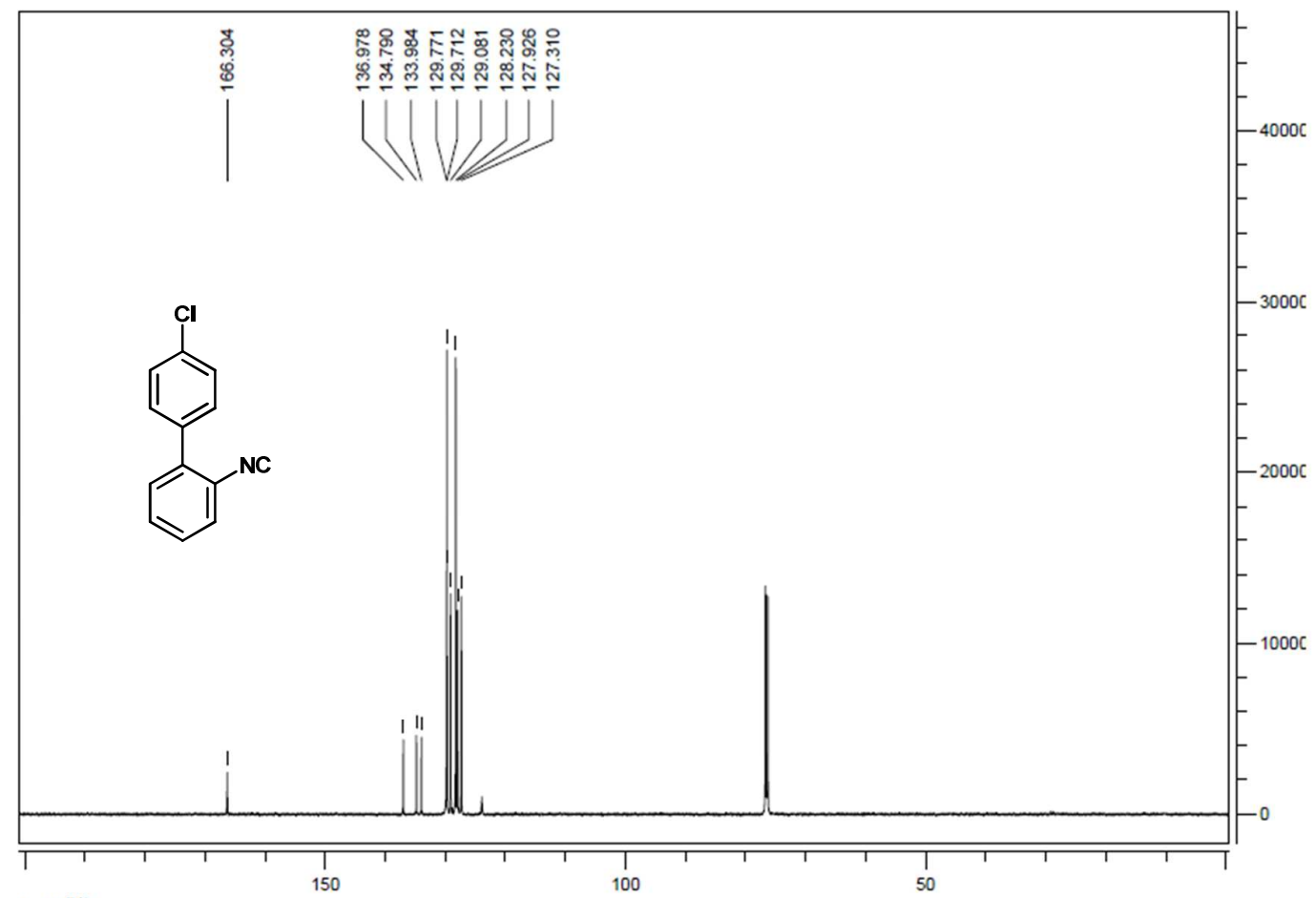

$\mathrm{ppm}(\mathrm{t} 1)$ 
2-isocyano-4'-(trifluoromethyl)-1,1'-biphenyl (1k)

Proton NMR $\left(\mathrm{CDCl}_{3}\right)$

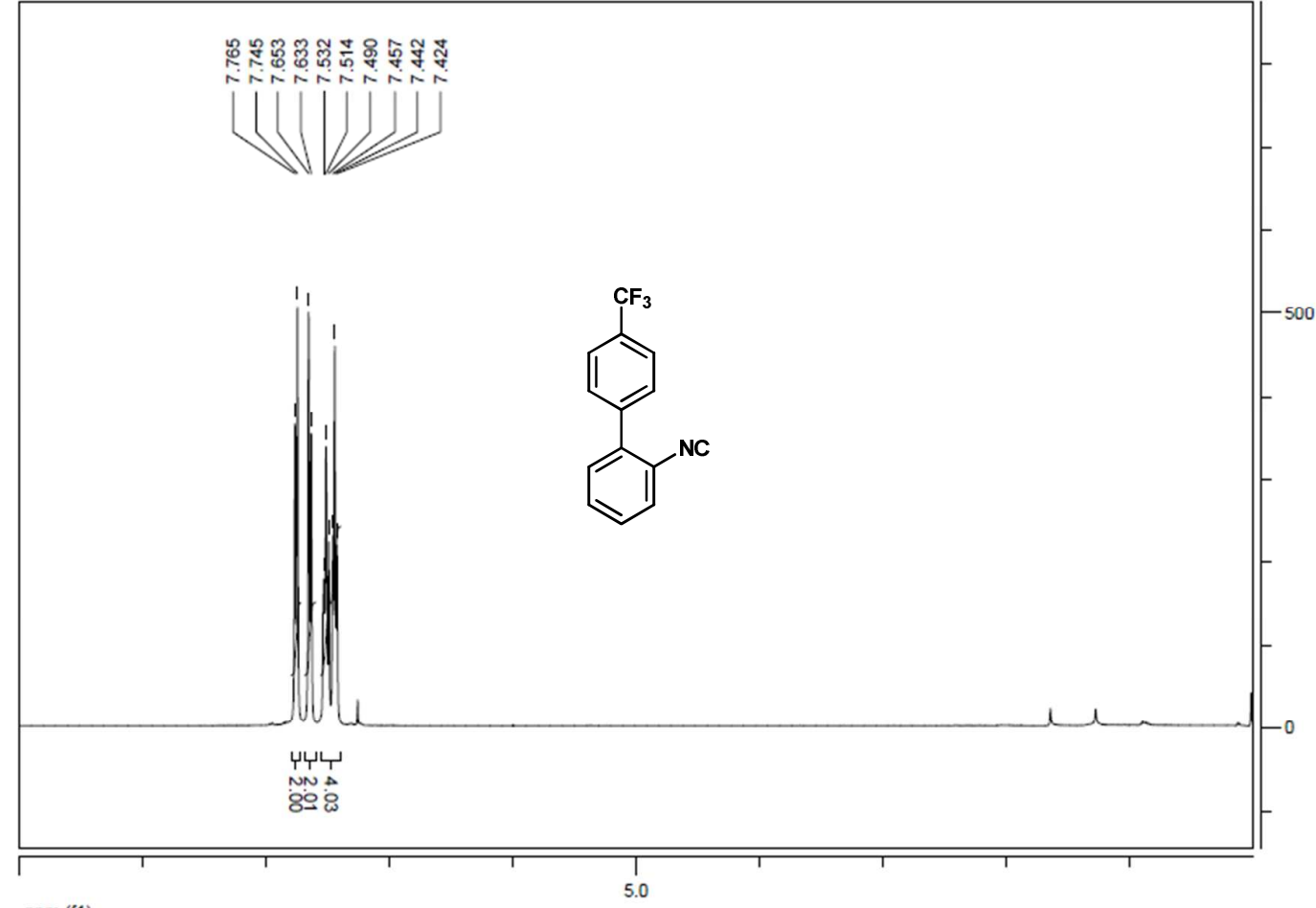

ppm (f1)

Carbon NMR ( $\left.\mathrm{CDCl}_{3}\right)$

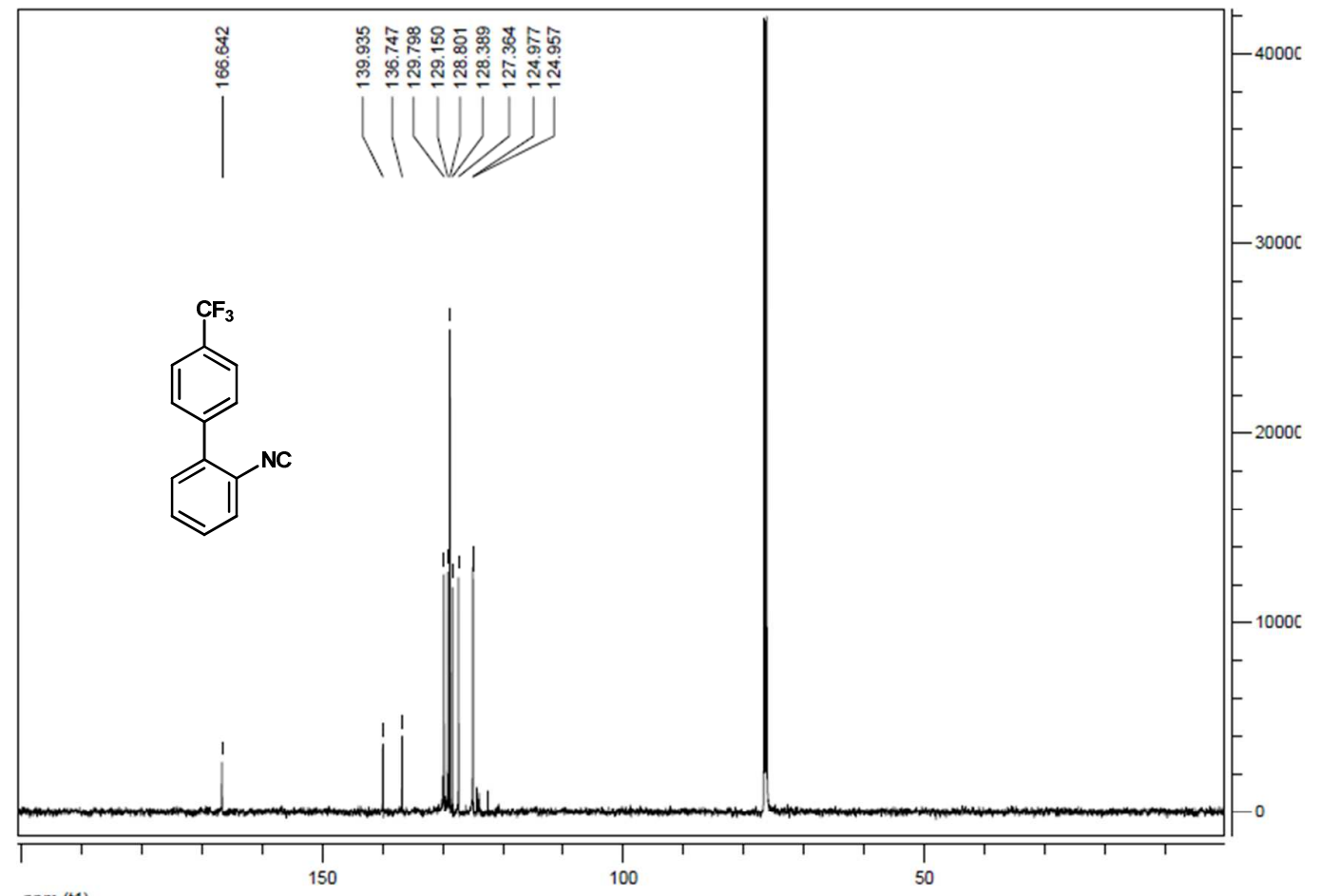

$\mathrm{ppm}(\mathrm{t} 1)$ 
2-isocyano-4-methyl-1,1'-biphenyl (11)

Proton NMR $\left(\mathrm{CDCl}_{3}\right)$

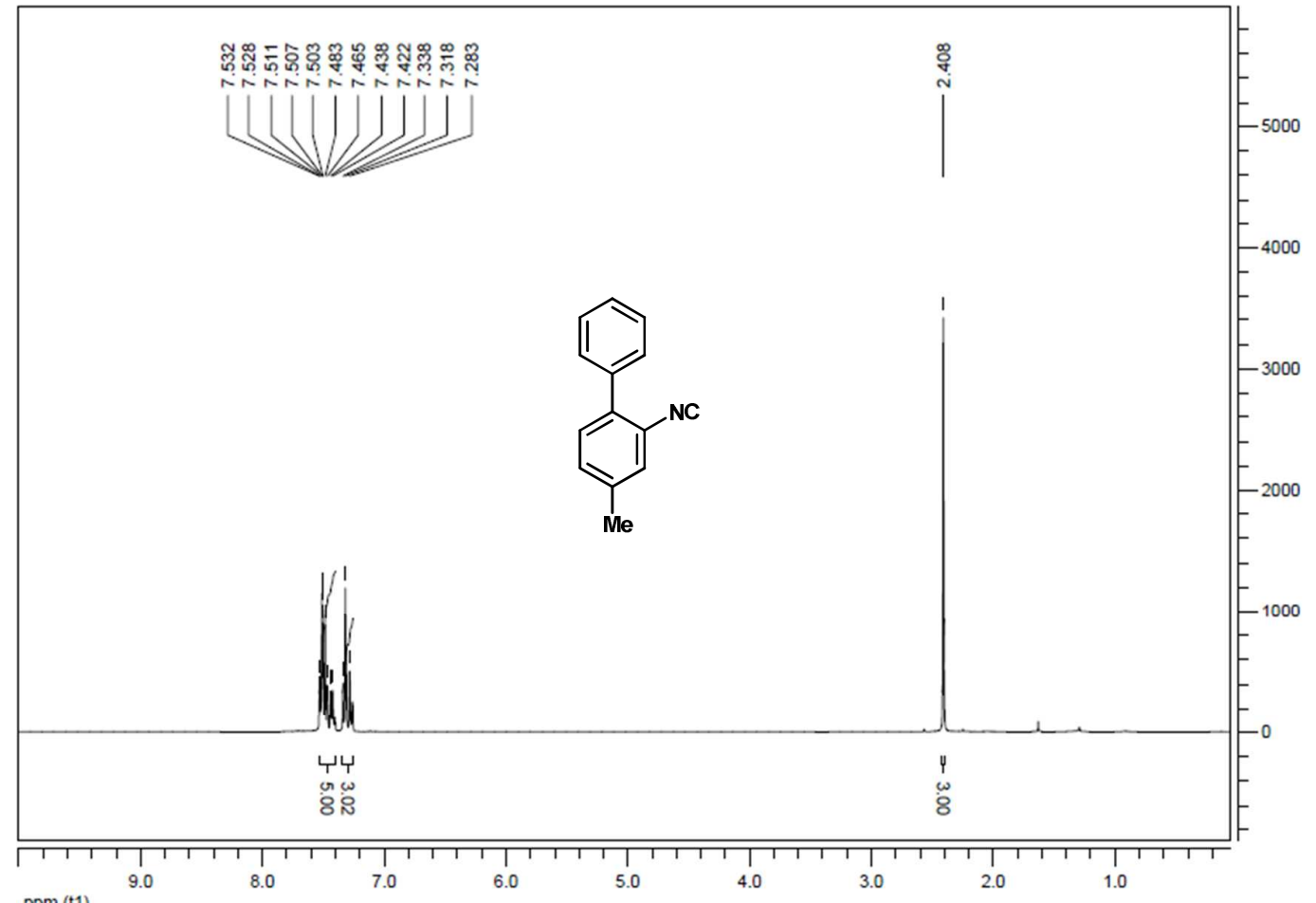

\section{Carbon NMR $\left(\mathrm{CDCl}_{3}\right)$}

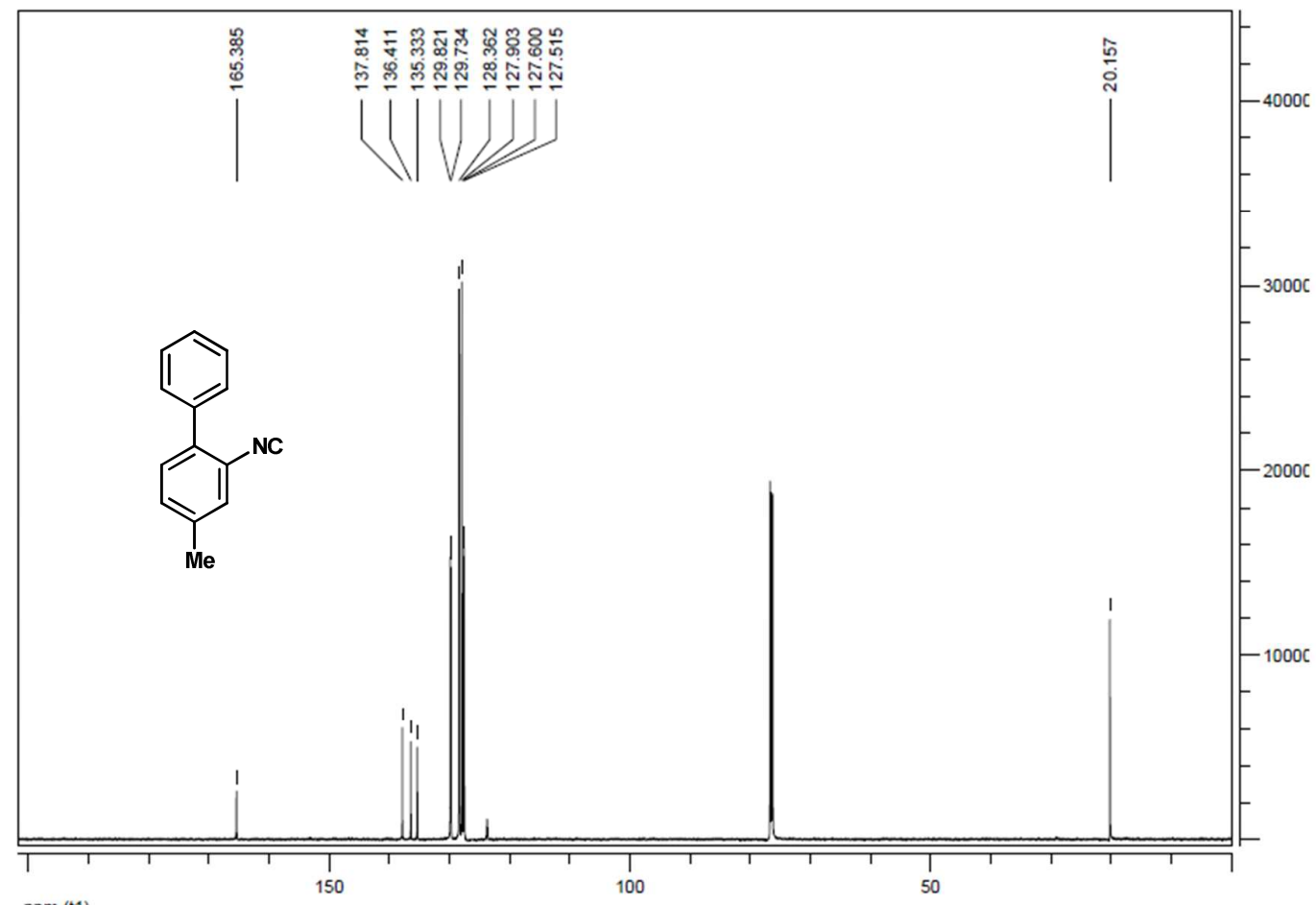

ppm (t1) 
2-isocyano-4-methoxy-1,1'-biphenyl (1m)

Proton NMR $\left(\mathrm{CDCl}_{3}\right)$

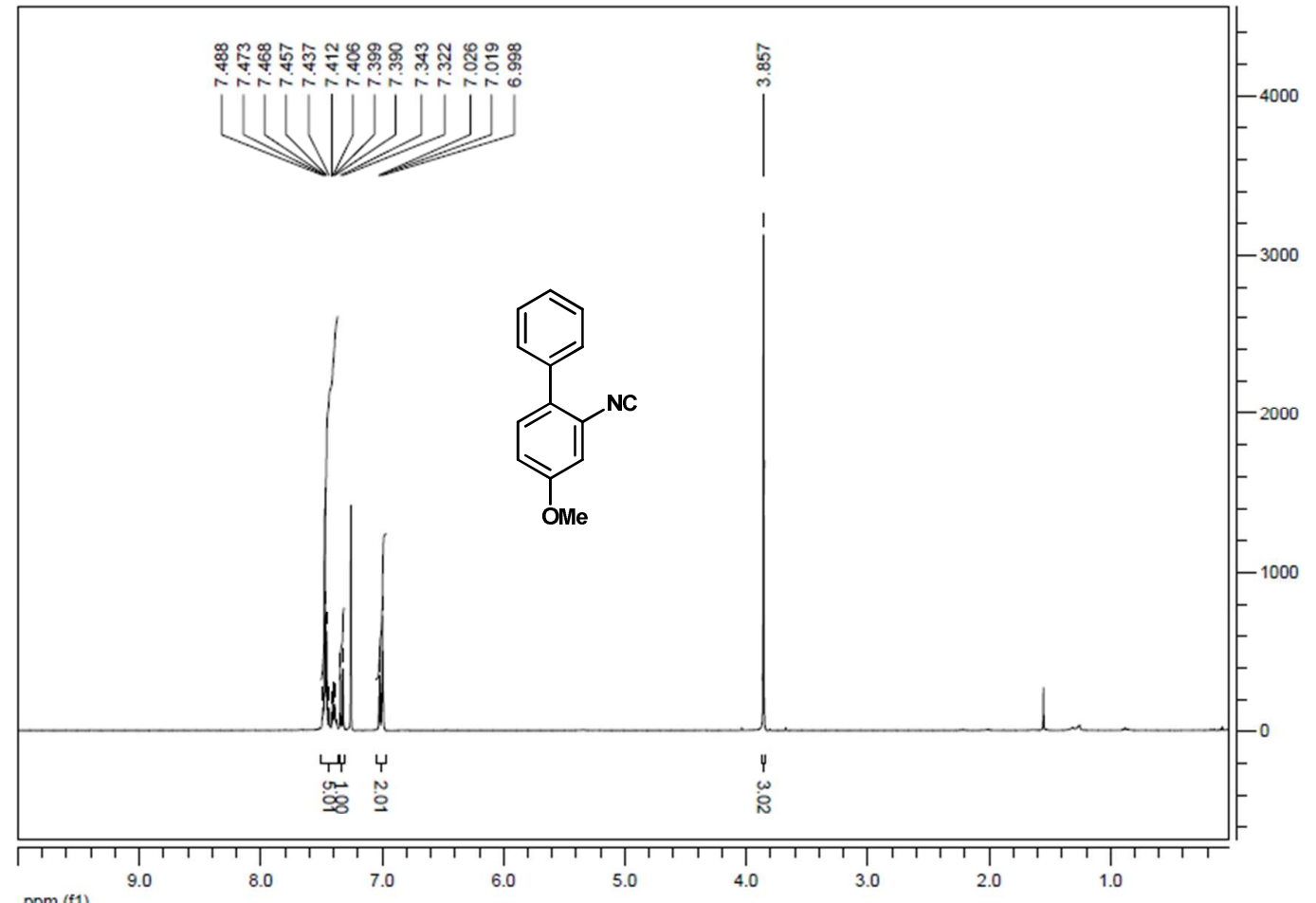

\section{Carbon NMR (CDCl $)$}

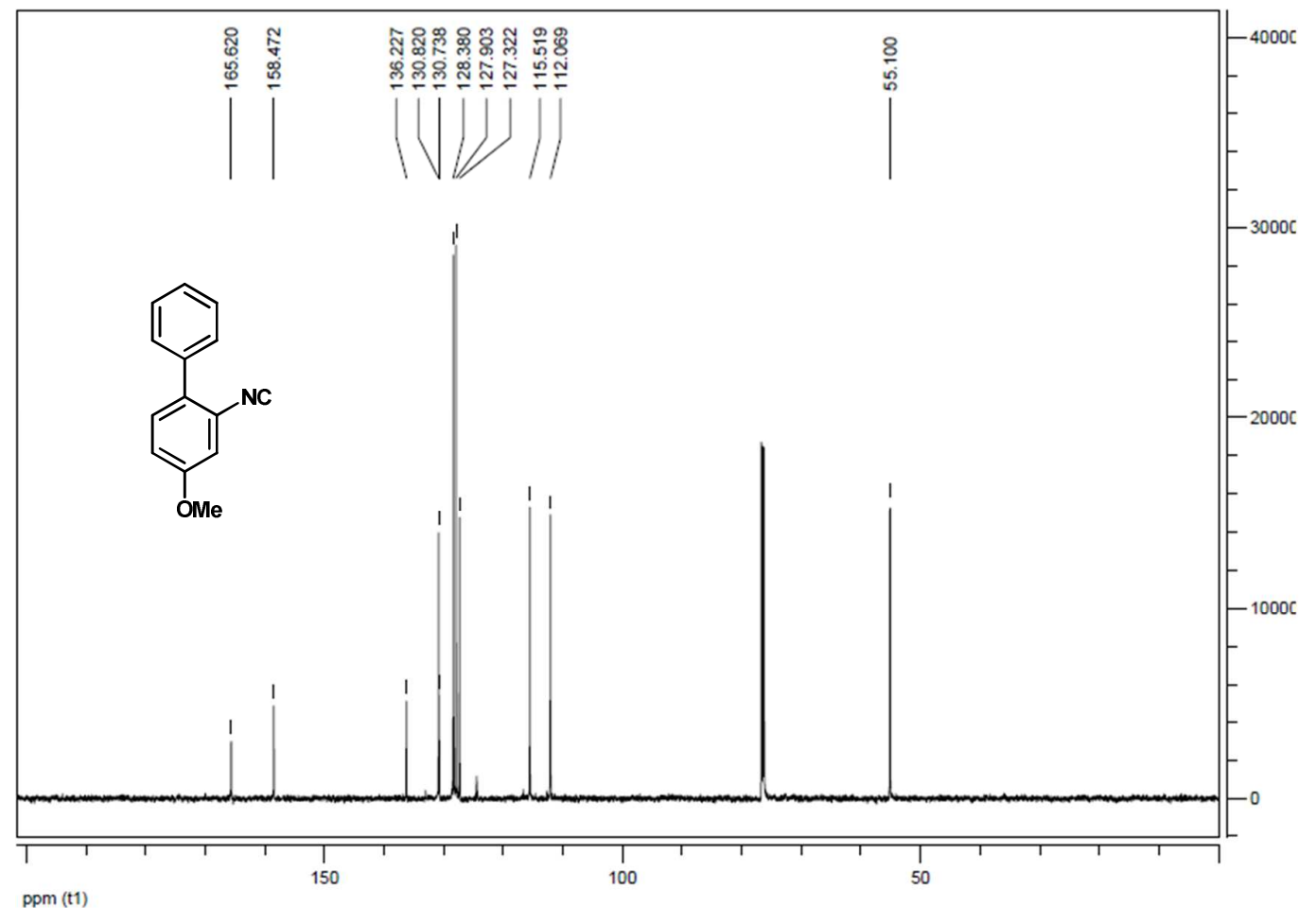


2-isocyano-3'-methyl-1,1'-biphenyl (1n)

Proton NMR $\left(\mathrm{CDCl}_{3}\right)$

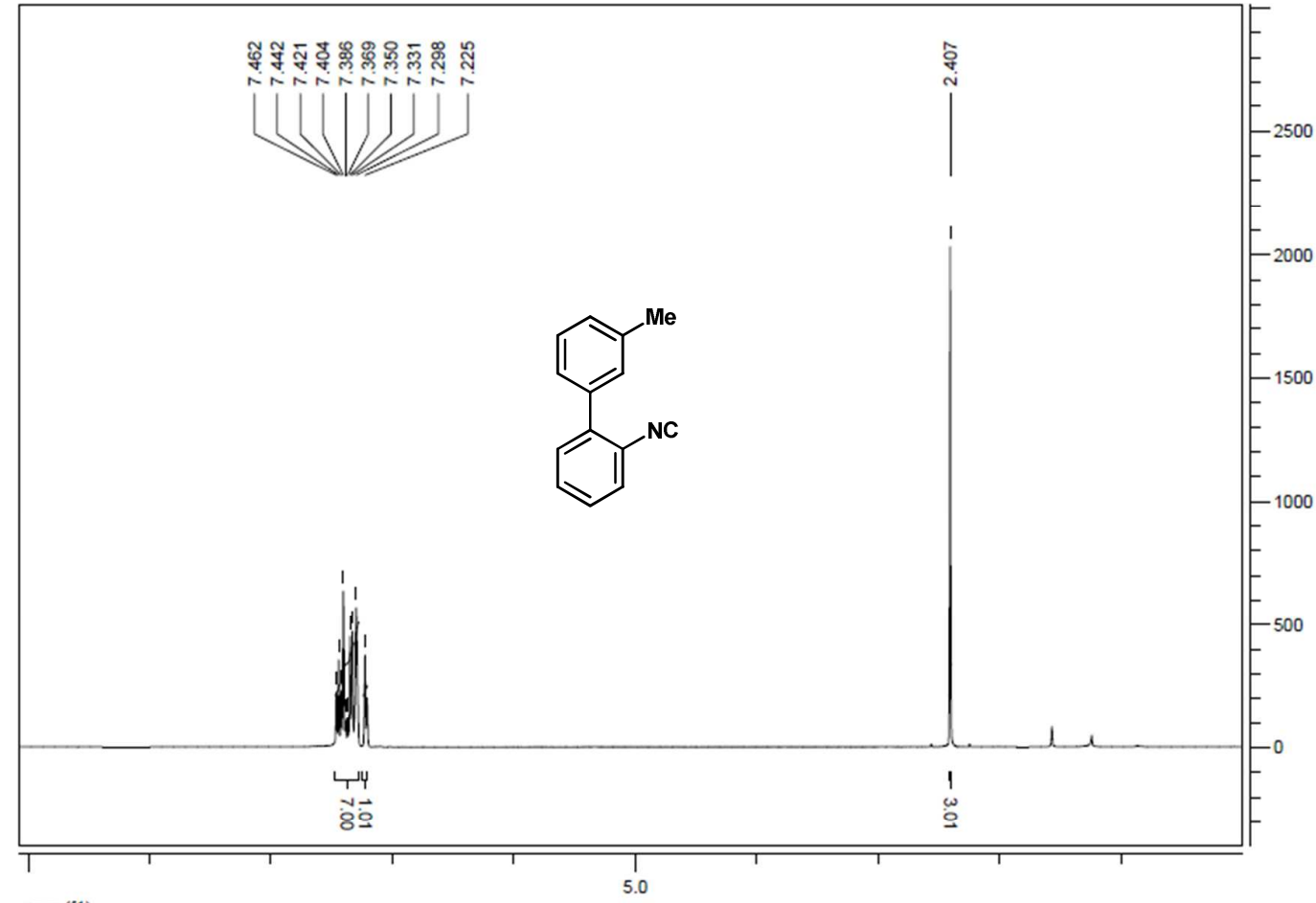

ppm (f1)

\section{Carbon NMR (CDCl $)_{3}$}

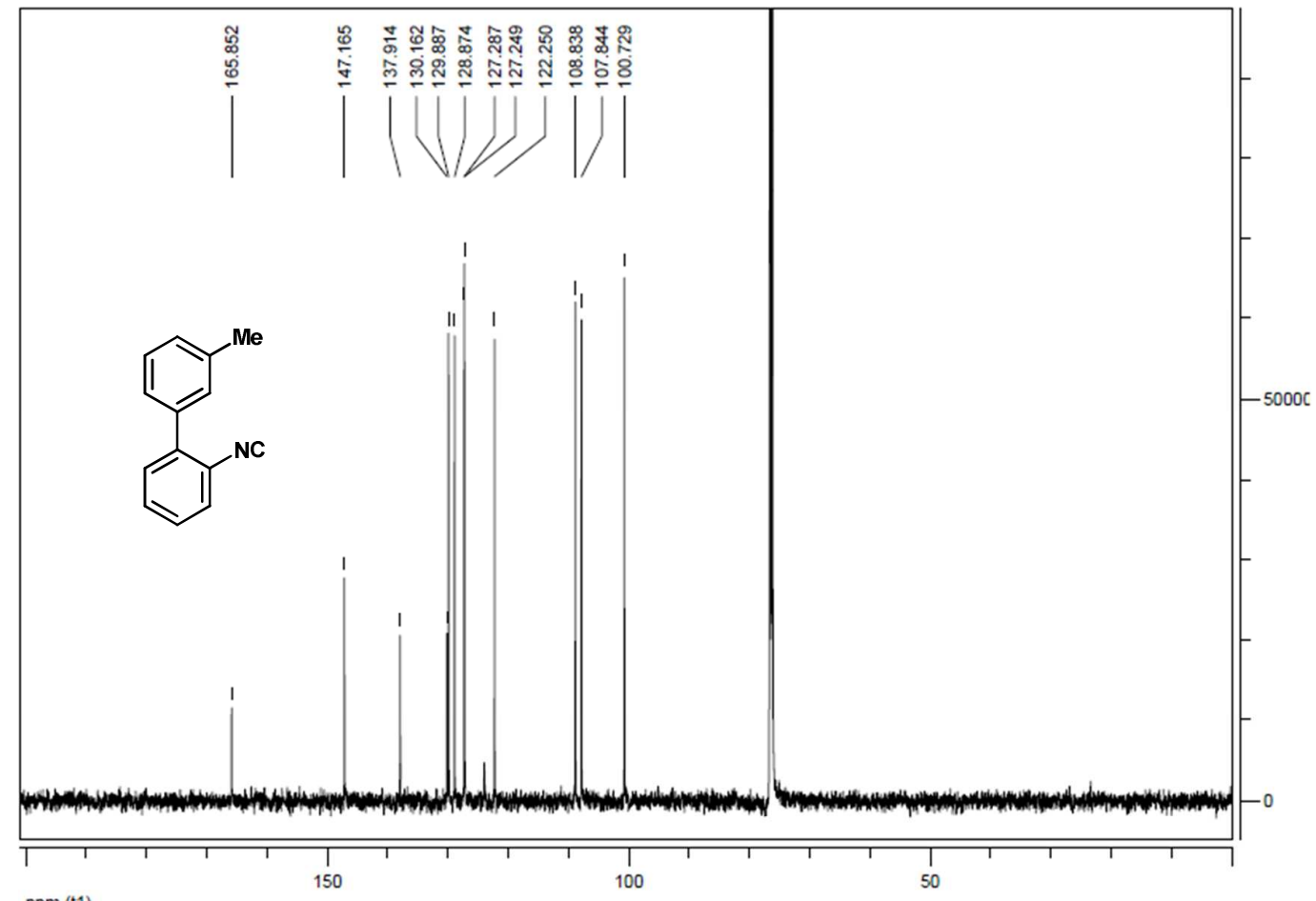

ppm (t1) 
2-isocyano-4',5-dimethyl-1,1'-biphenyl (10)

Proton NMR $\left(\mathrm{CDCl}_{3}\right)$

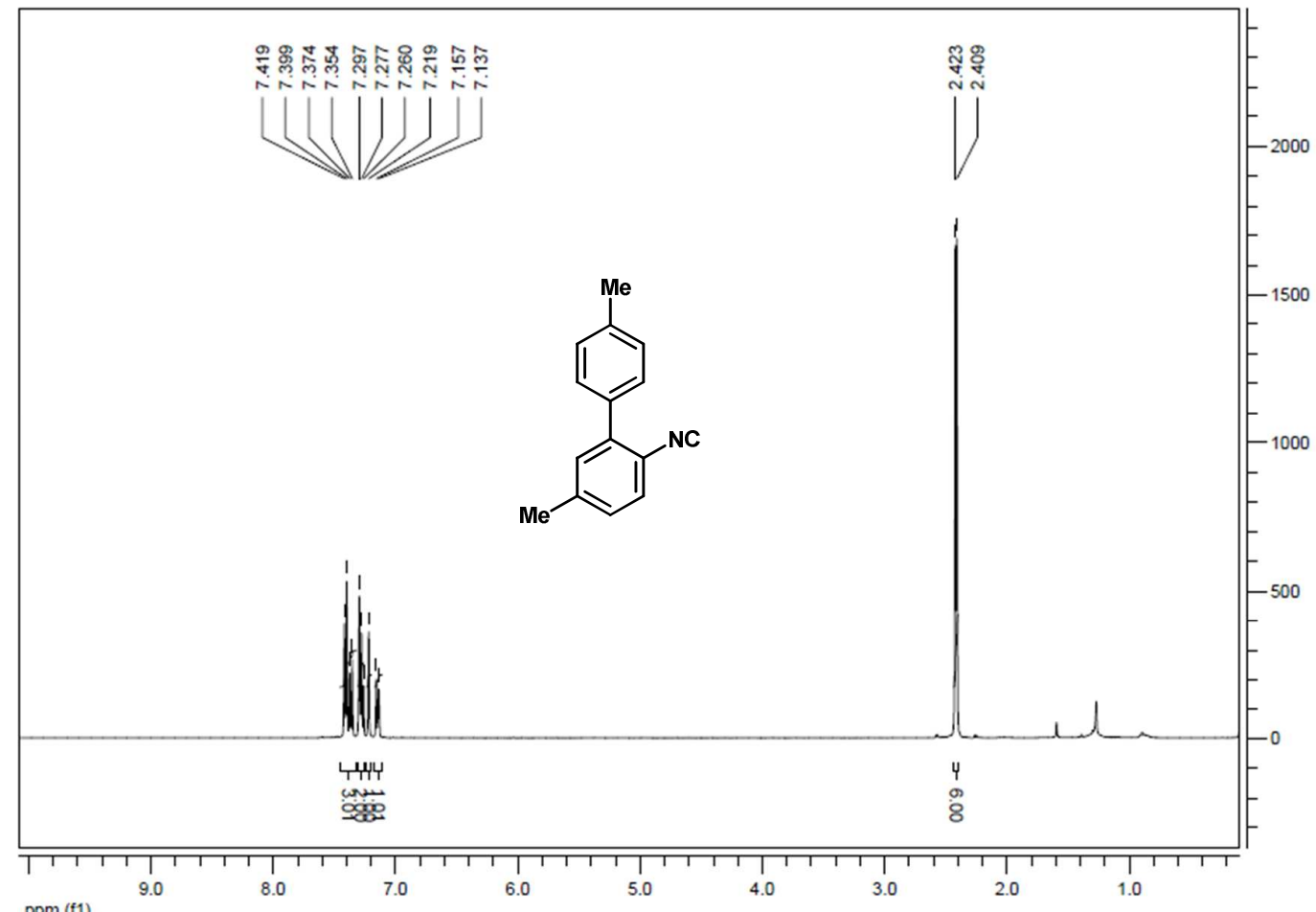

\section{Carbon NMR (CDCl $)_{3}$}

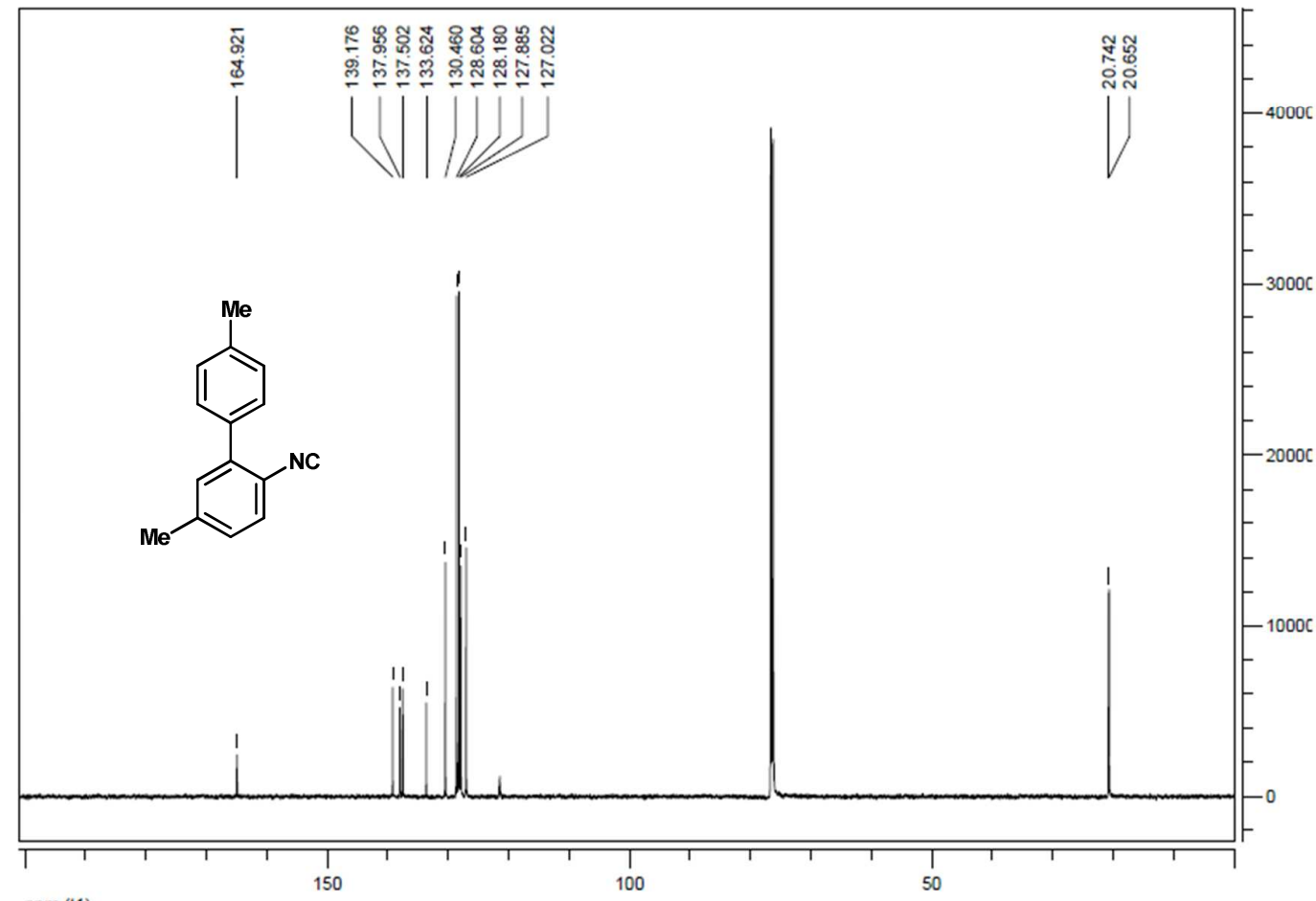

$\mathrm{ppm}(\mathrm{t} 1)$ 
4'-fluoro-2-isocyano-5-methyl-1,1'-biphenyl (1p)

Proton NMR $\left(\mathrm{CDCl}_{3}\right)$

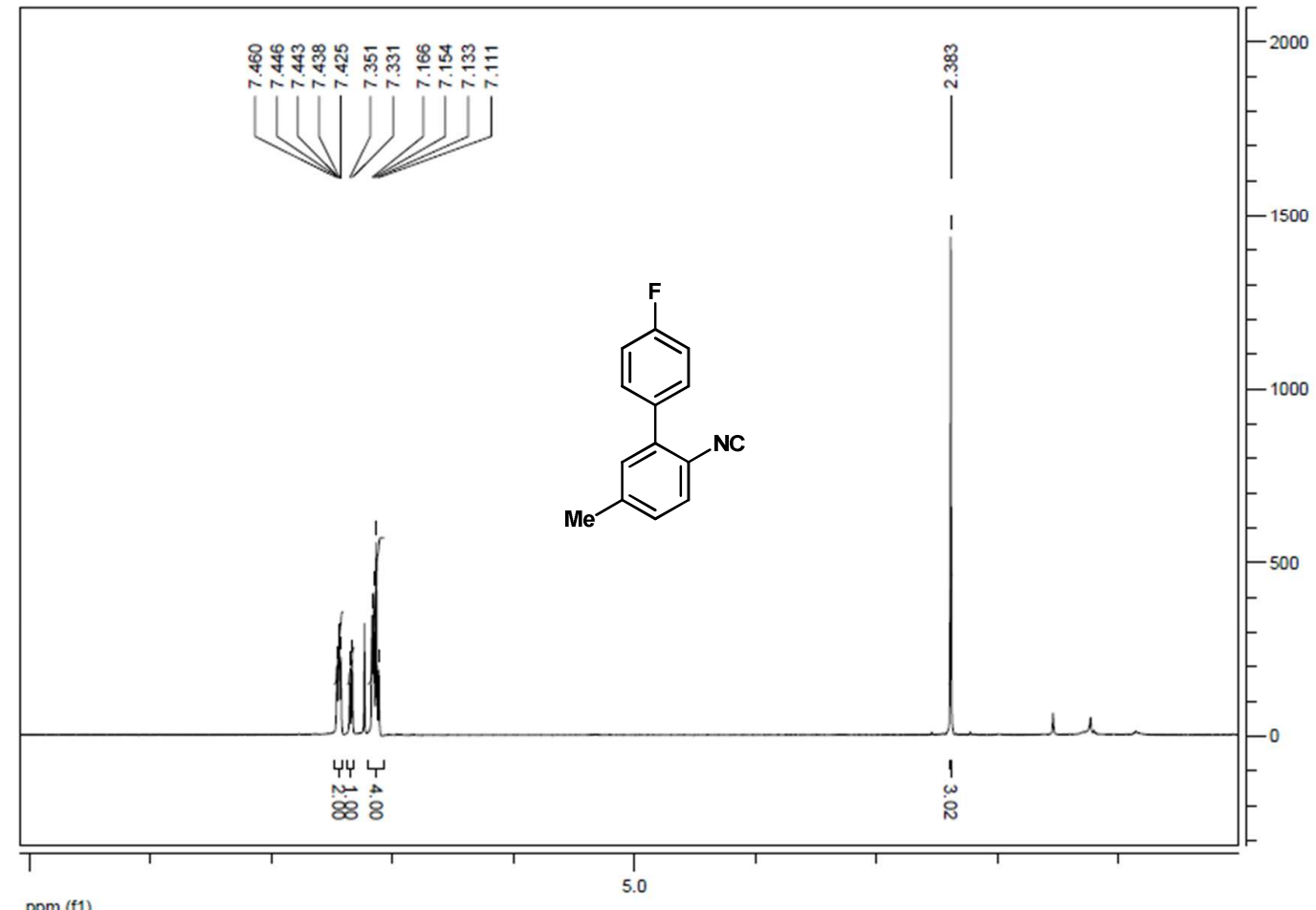

Carbon NMR (CDCl $\left.)_{3}\right)$

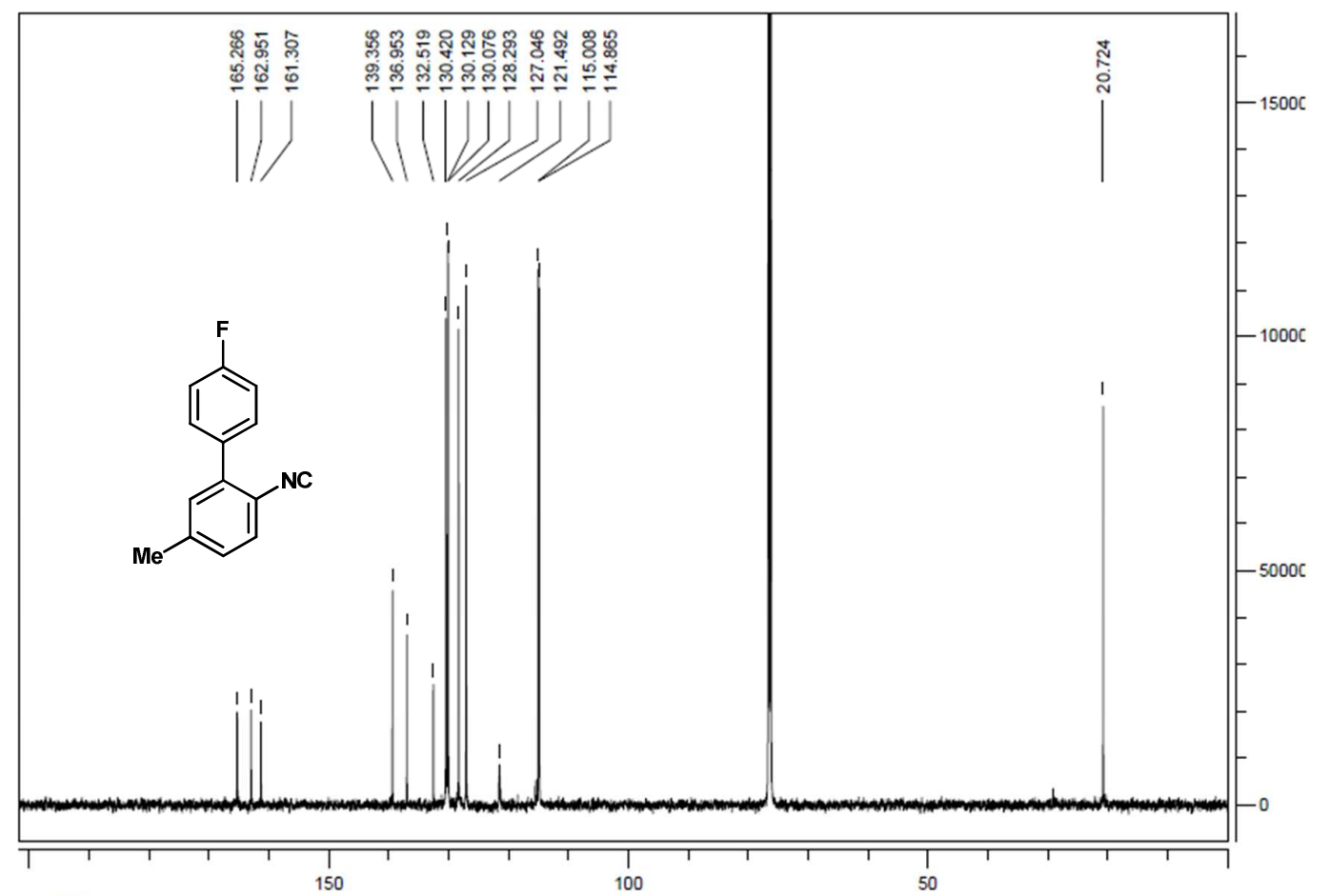

ppm (t1) 
5-(2-isocyano-5-methylphenyl)benzo $[d][1,3]$ dioxole (1q)

Proton NMR $\left(\mathrm{CDCl}_{3}\right)$

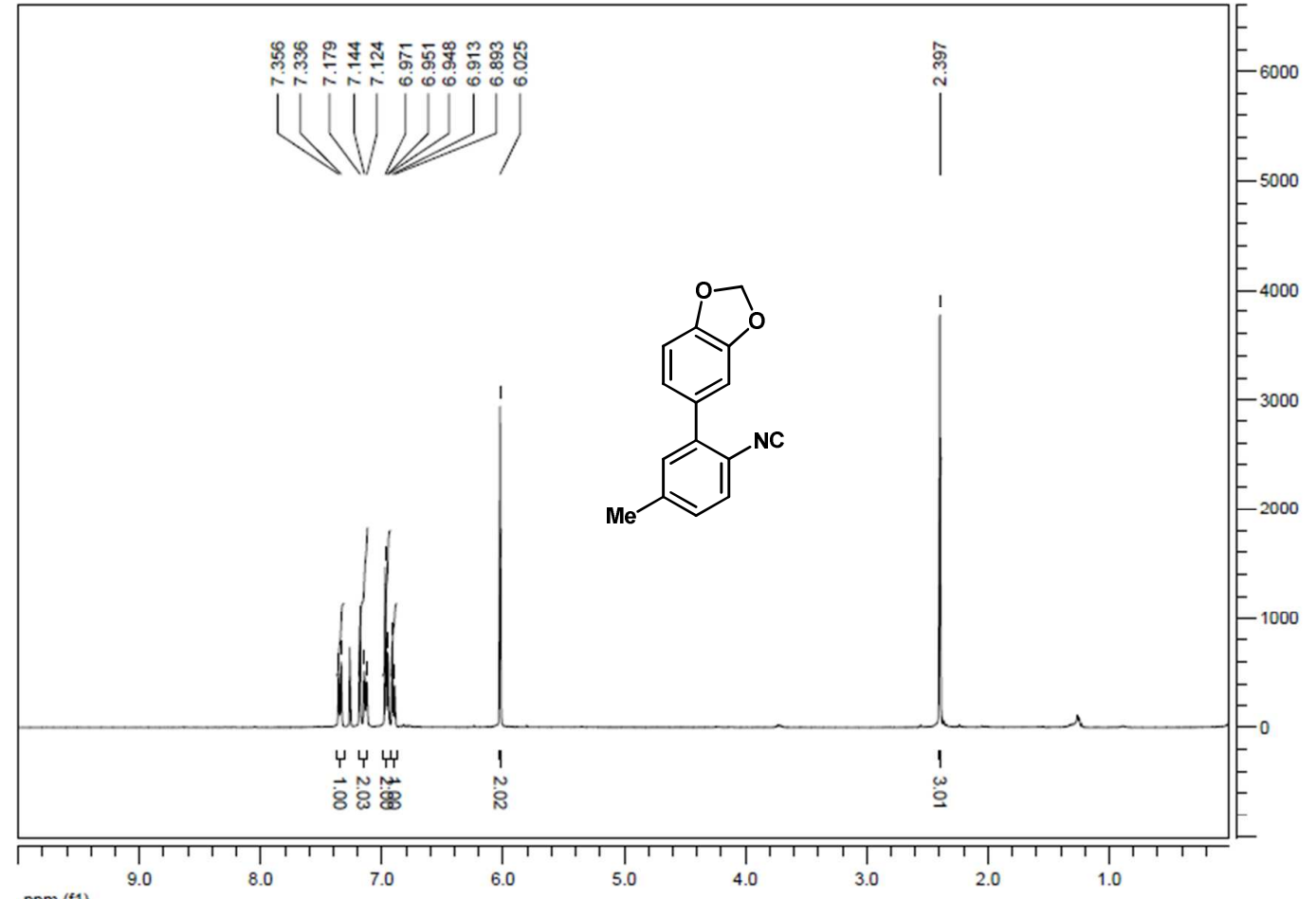

Carbon NMR $\left(\mathrm{CDCl}_{3}\right)$

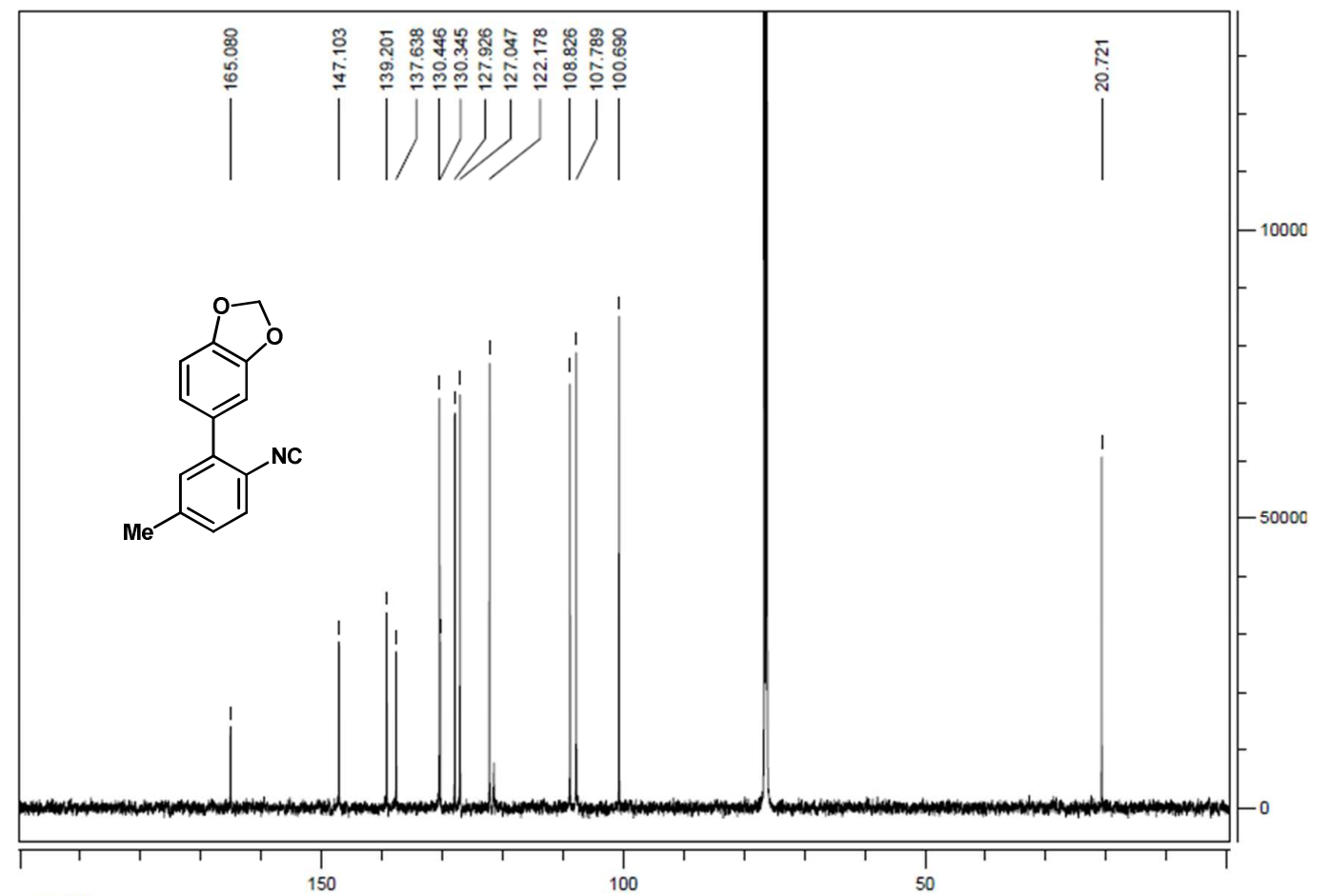

ppm (t1) 
6-(1-ethoxyethyl)phenanthridine (3a)

Proton NMR $\left(\mathrm{CDCl}_{3}\right)$

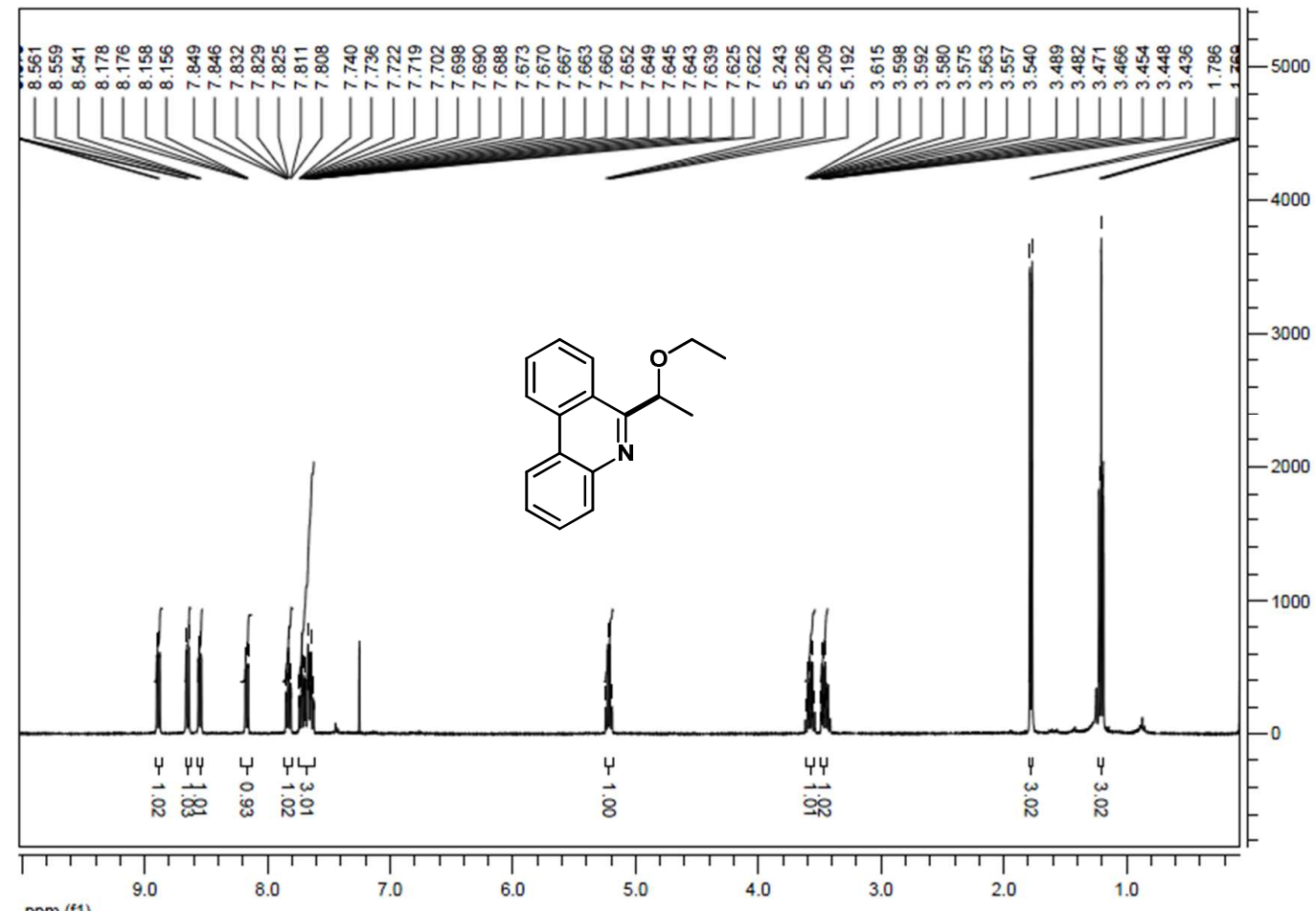

Carbon NMR $\left(\mathrm{CDCl}_{3}\right)$

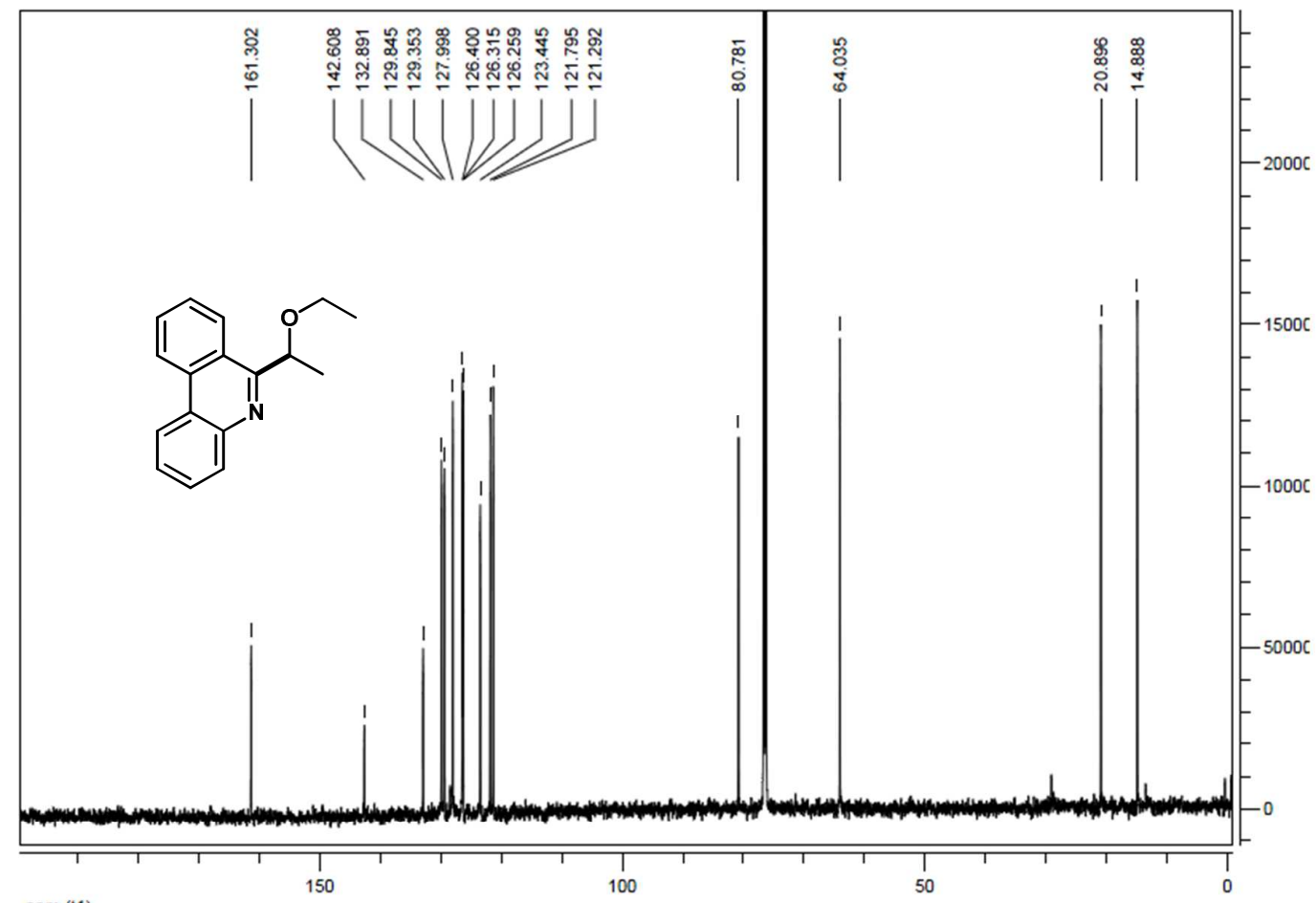

$\mathrm{ppm}(\mathrm{t} 1)$ 
6-(1-ethoxyethyl)-2-methylphenanthridine (3b)

Proton NMR $\left(\mathrm{CDCl}_{3}\right)$

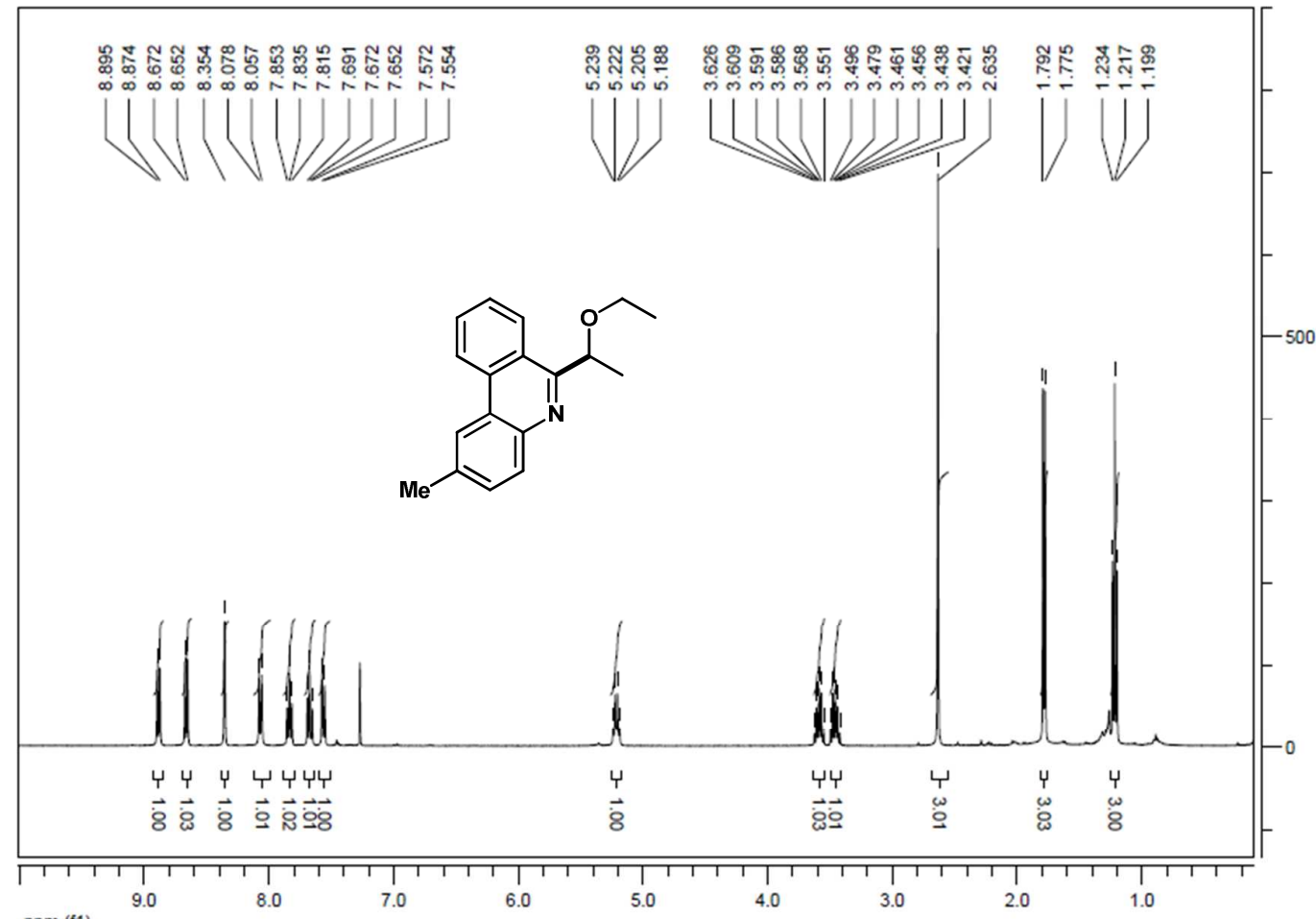

\section{Carbon NMR (CDCl $)_{3}$}

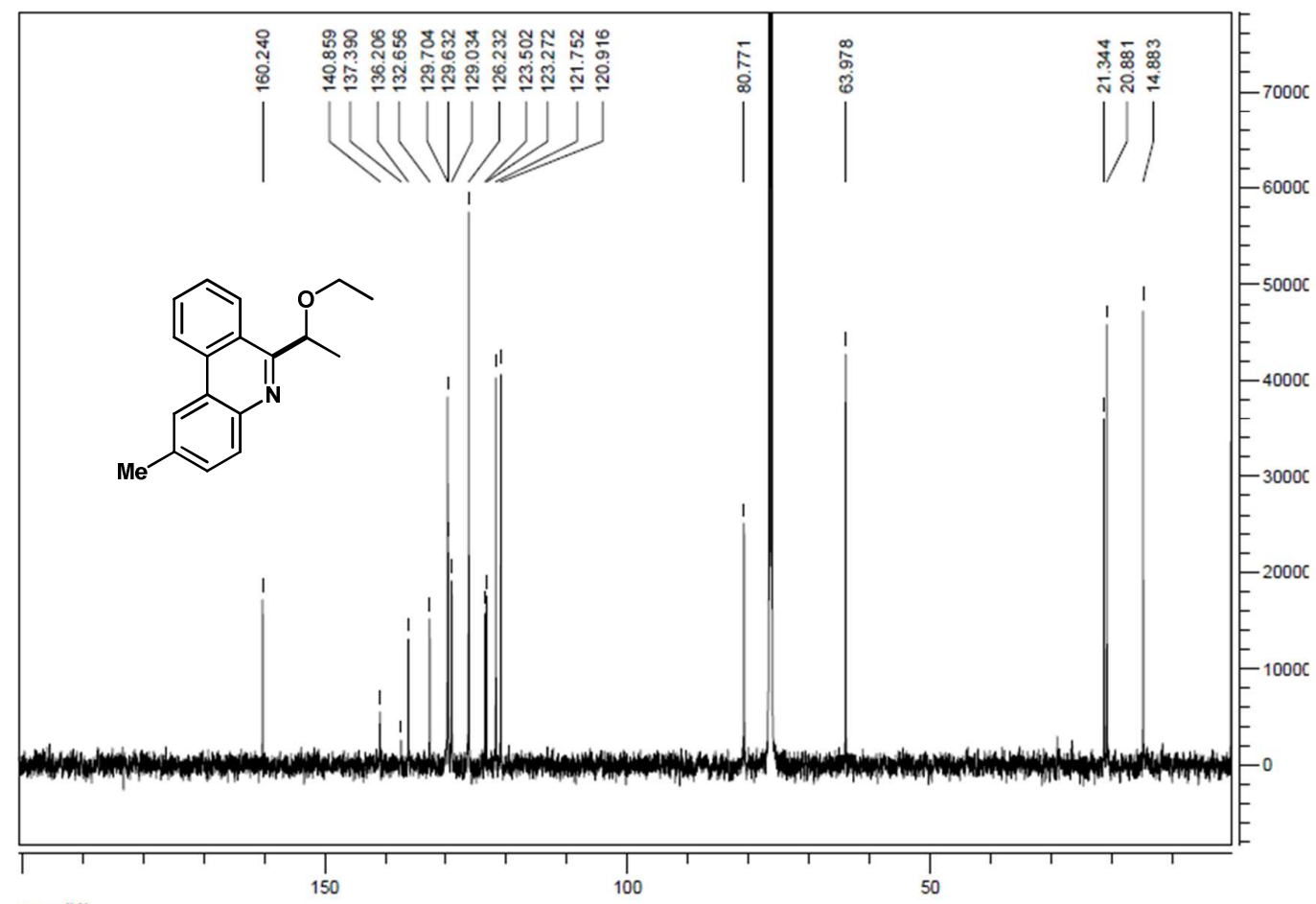

ppm (t1) 
6-(1-ethoxyethyl)-2-methoxyphenanthridine (3c)

Proton NMR $\left(\mathrm{CDCl}_{3}\right)$

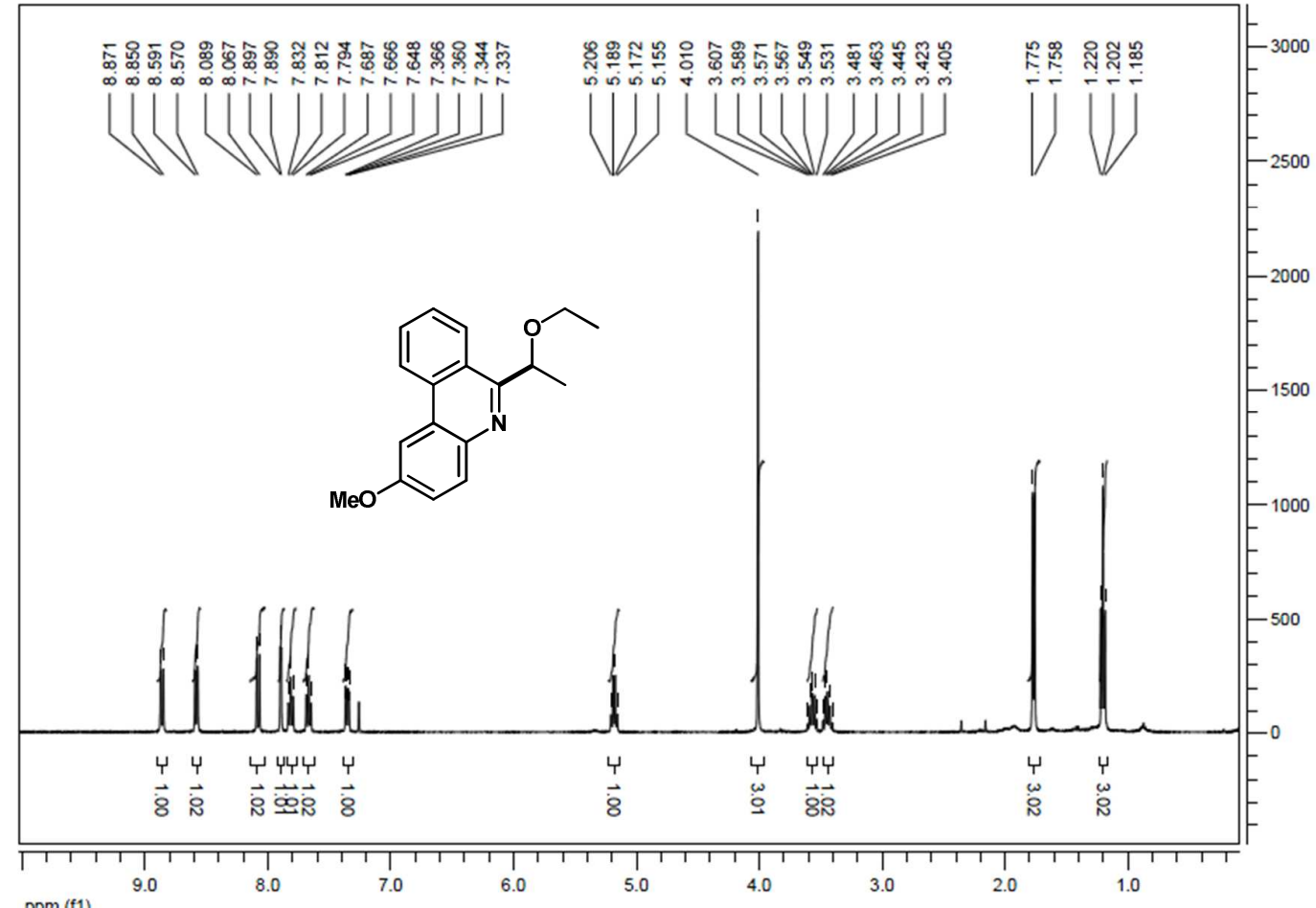

Carbon NMR ( $\left.\mathrm{CDCl}_{3}\right)$

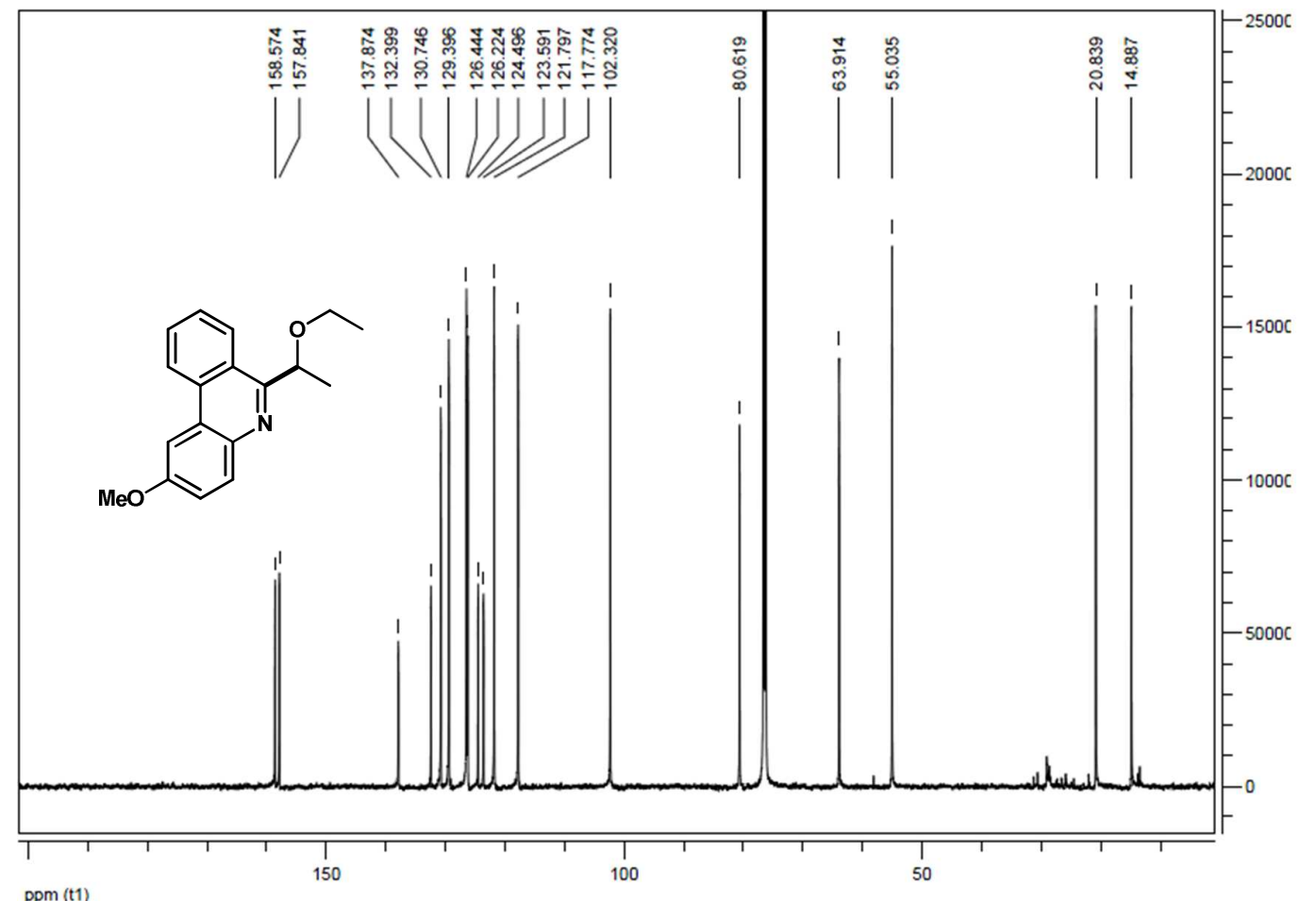


6-(1-ethoxyethyl)-2-fluorophenanthridine (3d)

Proton NMR $\left(\mathrm{CDCl}_{3}\right)$

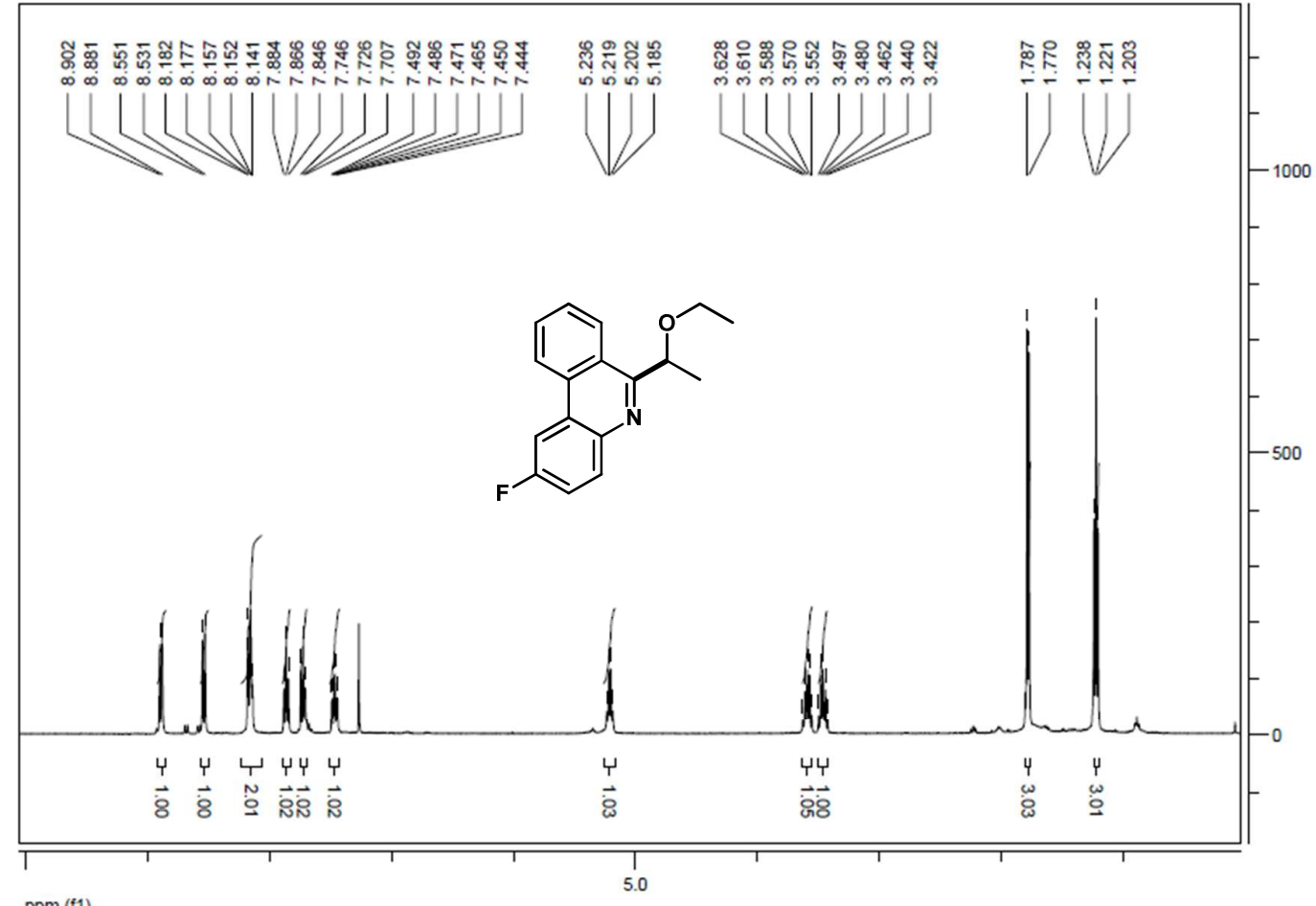

Carbon NMR (CDCl$\left.)_{3}\right)$

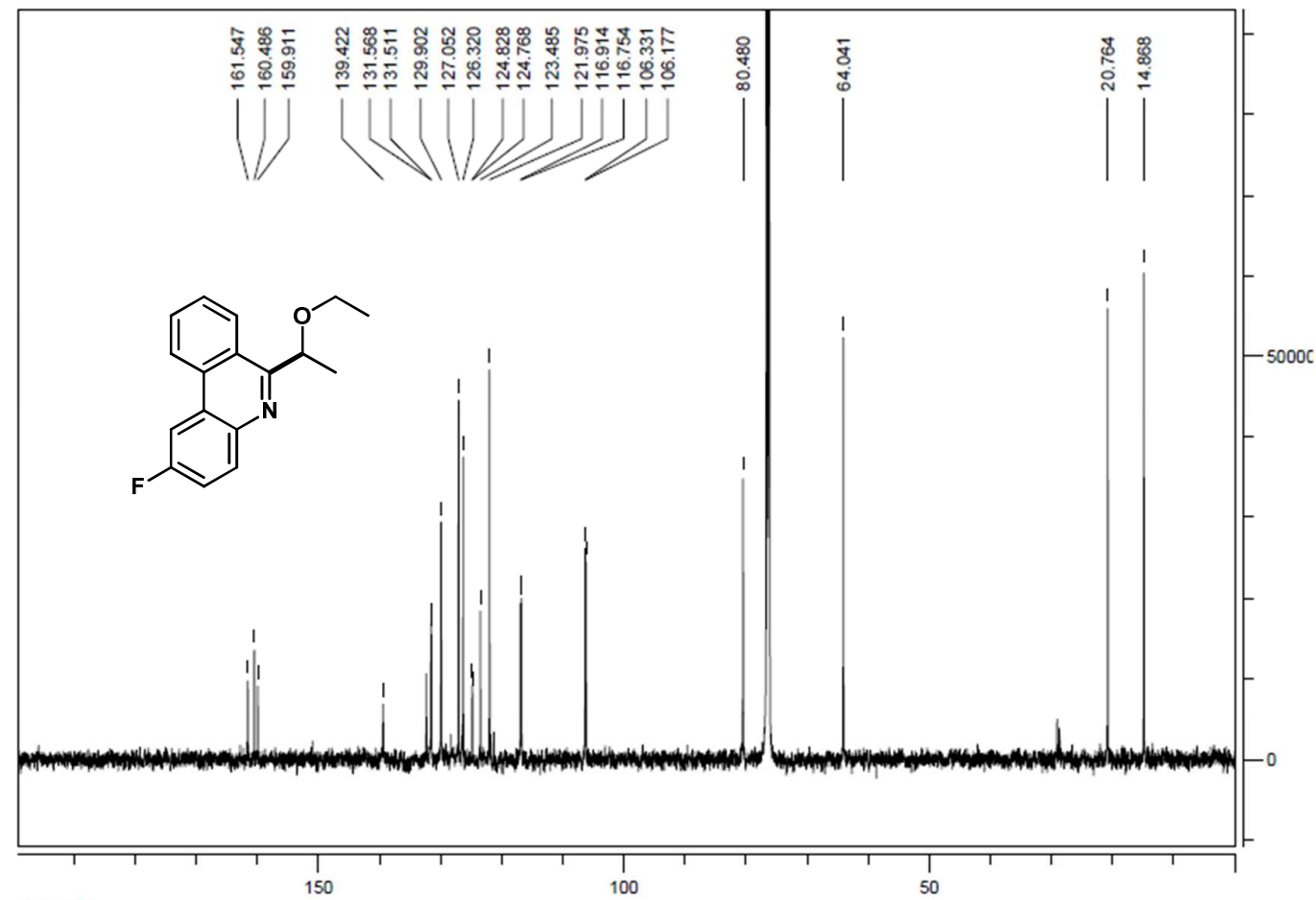

ppm (t1) 
2-chloro-6-(1-ethoxyethyl)phenanthridine (3e)

Proton NMR $\left(\mathrm{CDCl}_{3}\right)$

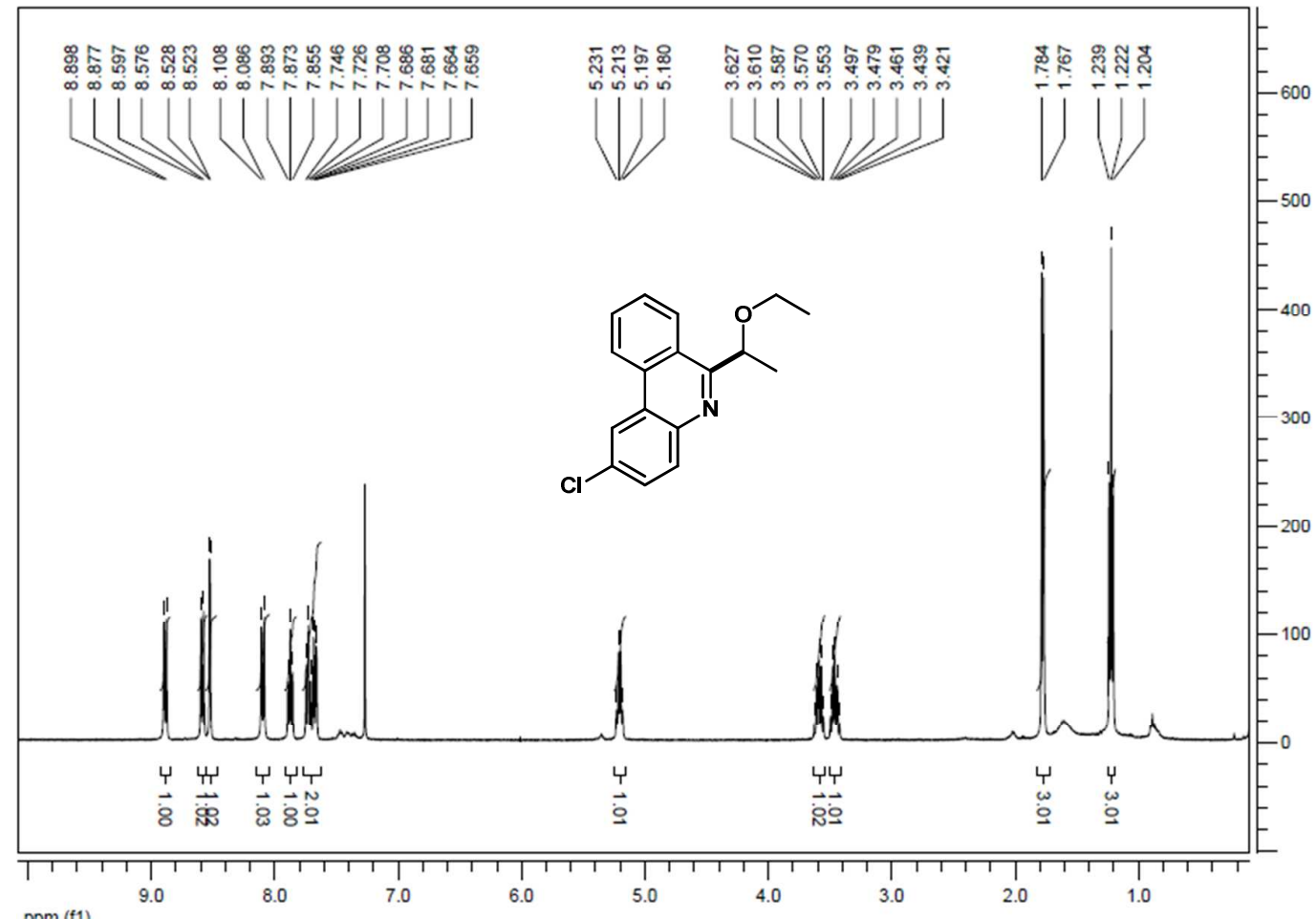

ppm (f1)

Carbon NMR ( $\left.\mathrm{CDCl}_{3}\right)$

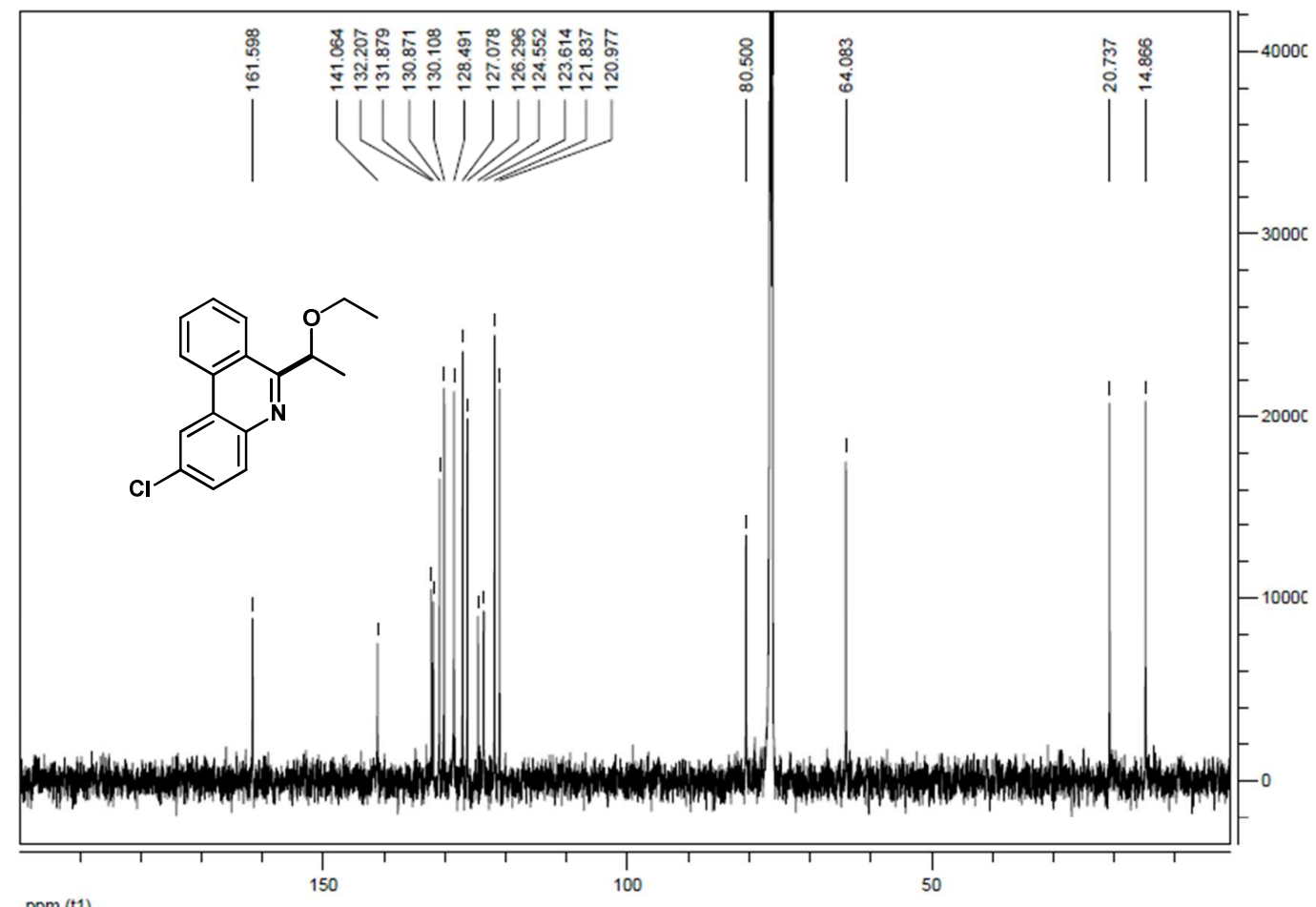

ppm (t1) 
6-(1-ethoxyethyl)-2-(trifluoromethyl)phenanthridine (3f)

Proton NMR $\left(\mathrm{CDCl}_{3}\right)$

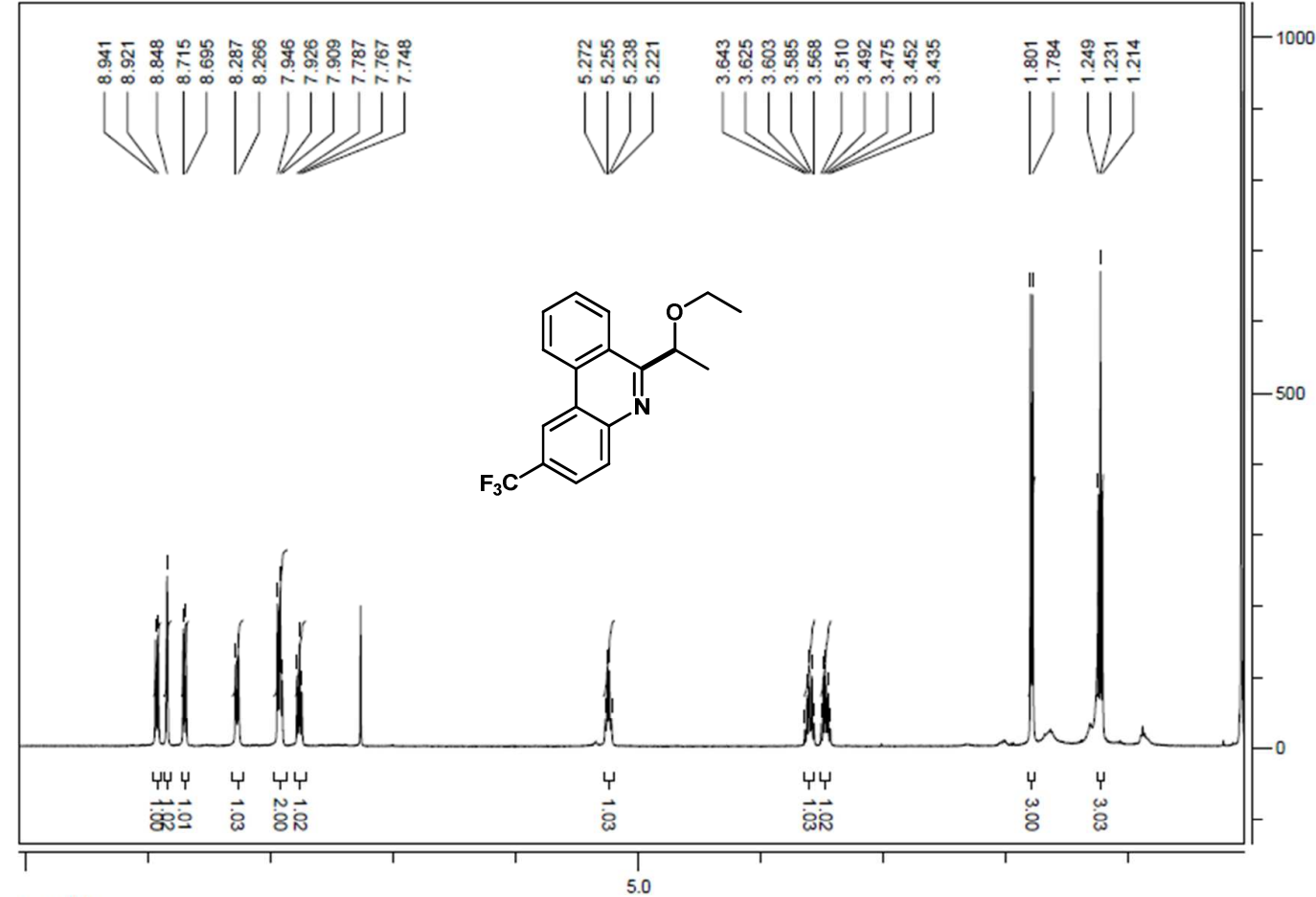

ppm (f1)

\section{Carbon NMR (CDCl $)_{3}$}

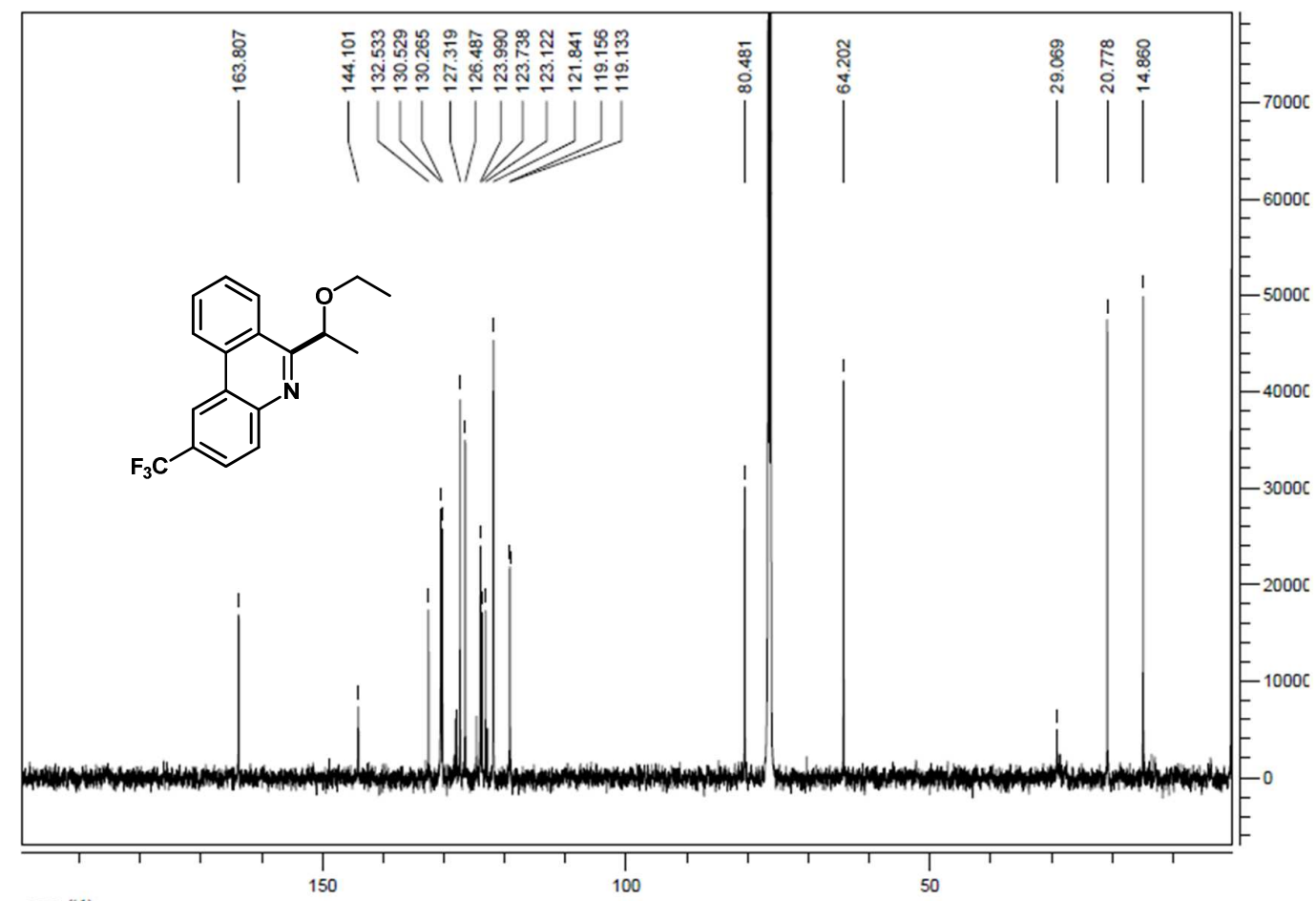

ppm (t1) 
6-(1-ethoxyethyl)-8-methylphenanthridine (3g)

Proton NMR $\left(\mathrm{CDCl}_{3}\right)$

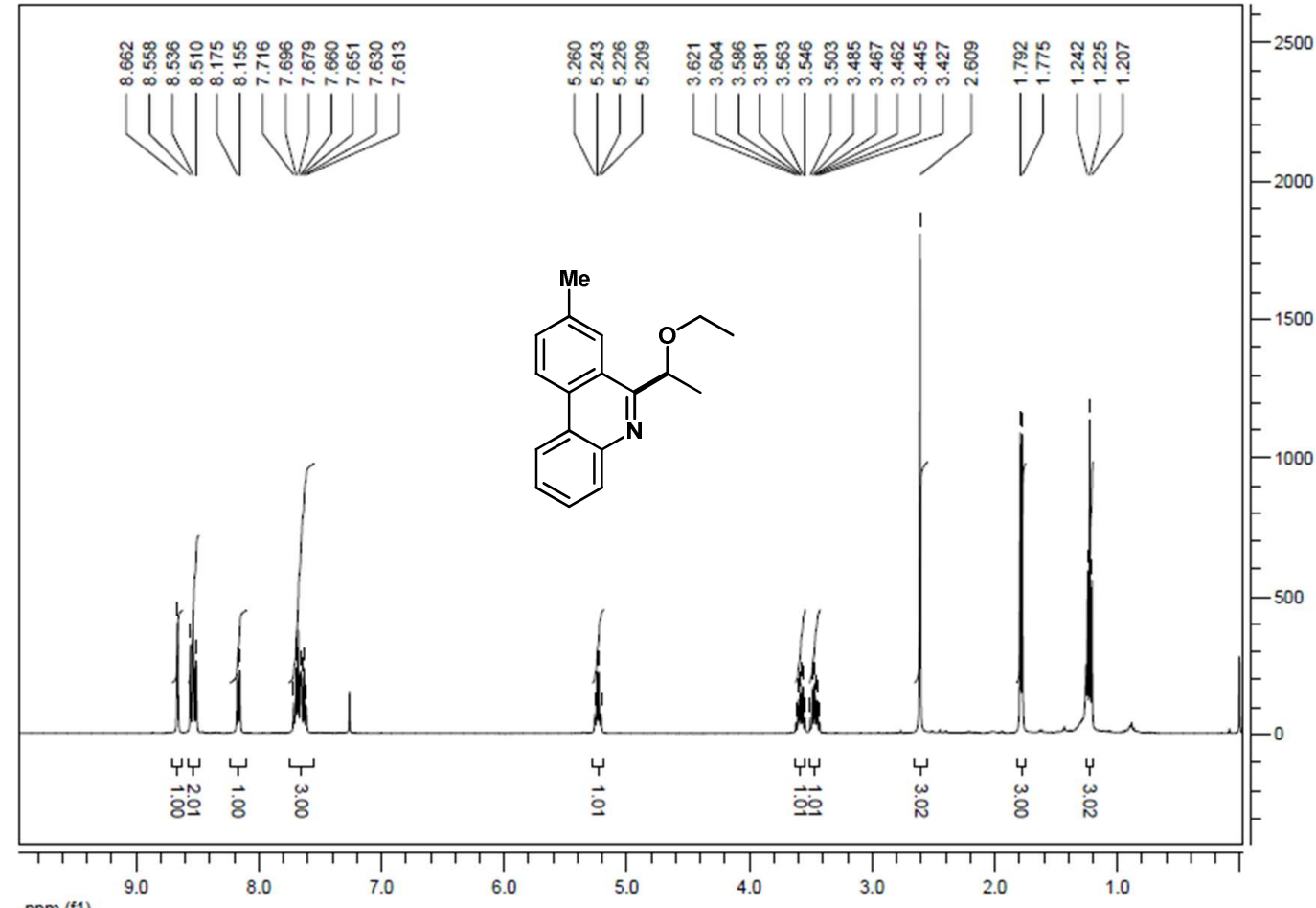

\section{Carbon NMR (CDCl $)_{3}$}

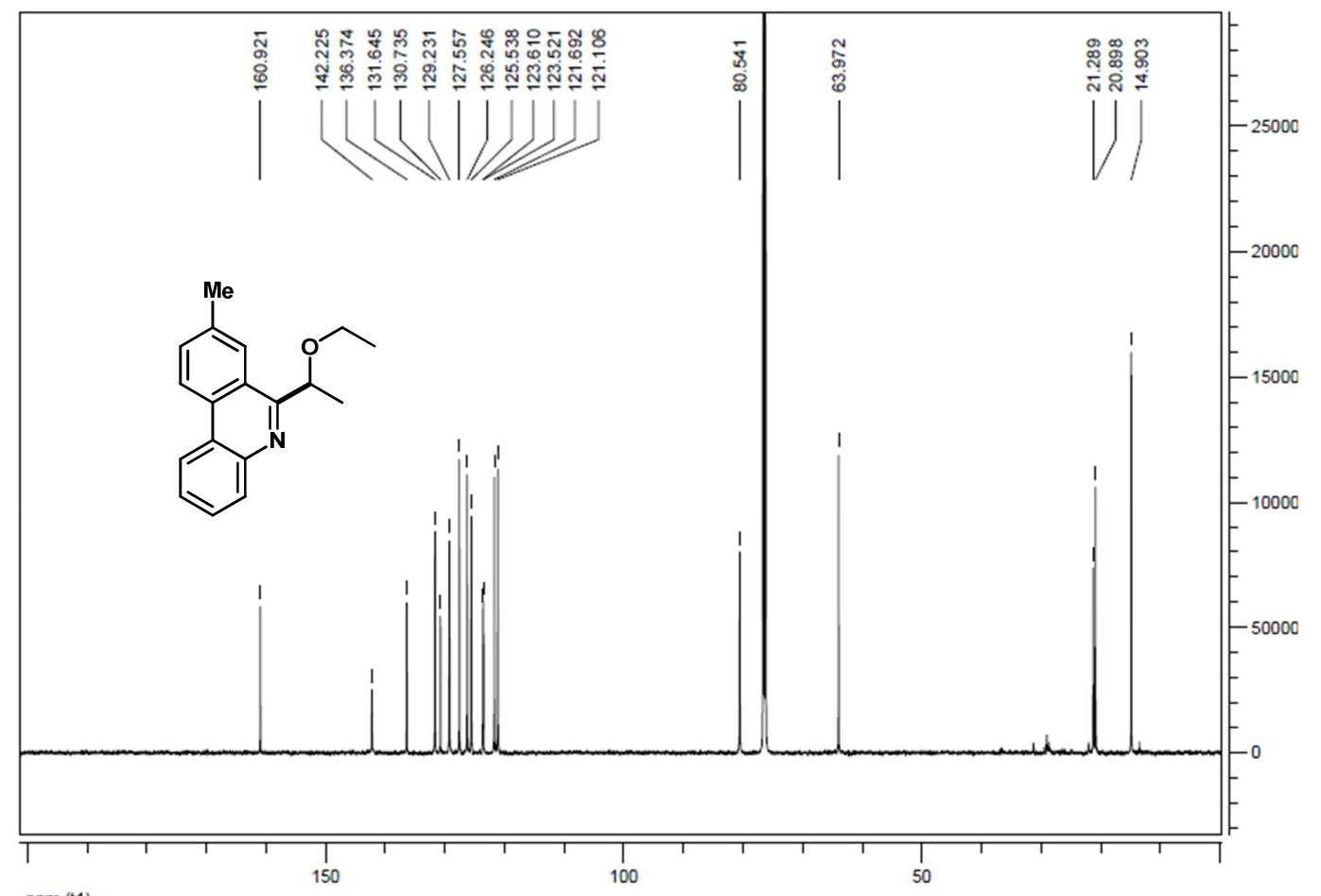

ppm (t1) 
6-(1-ethoxyethyl)-8-methoxyphenanthridine (3h)

Proton NMR $\left(\mathrm{CDCl}_{3}\right)$

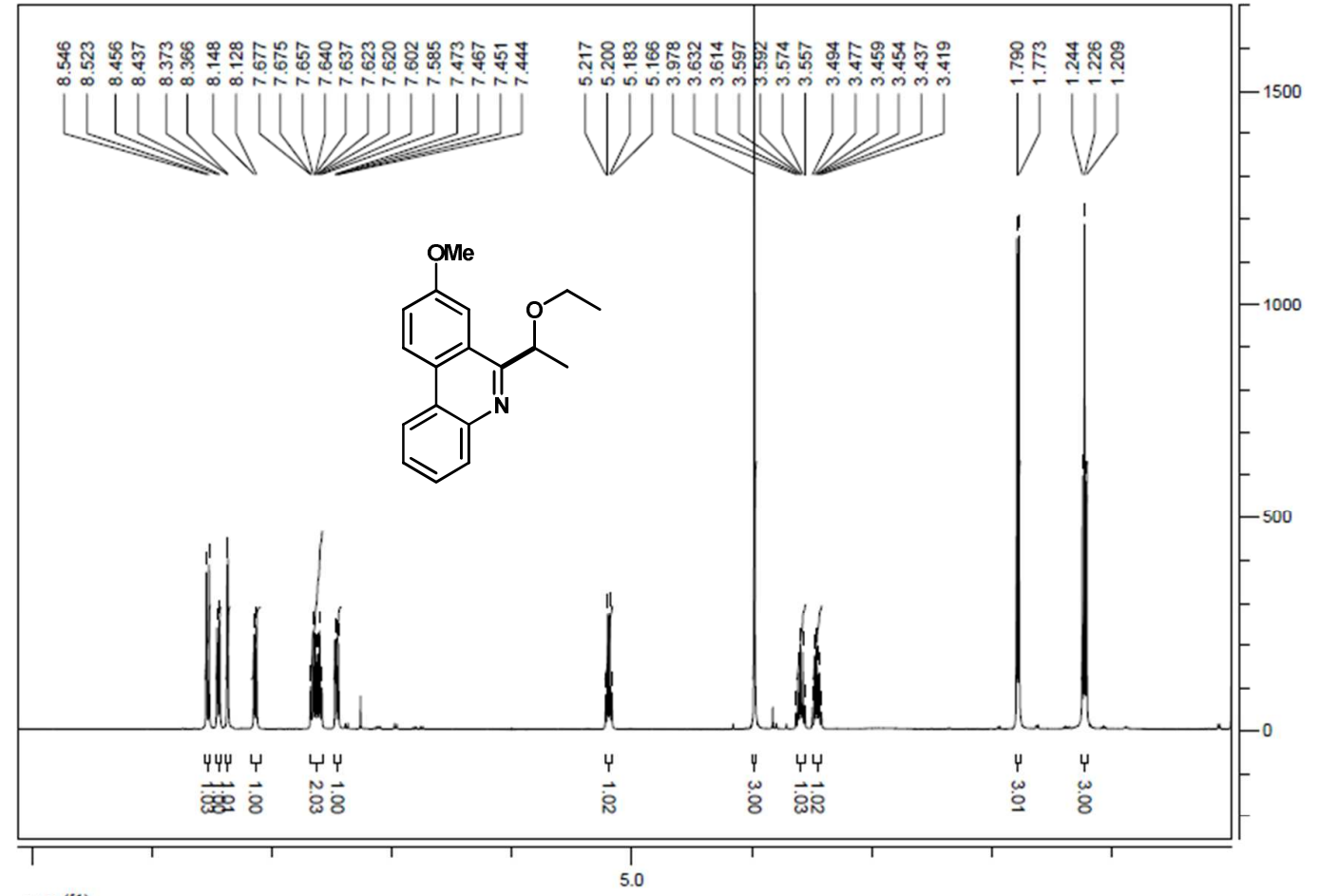

ppm (f1)

\section{Carbon NMR (CDCl $)_{3}$}

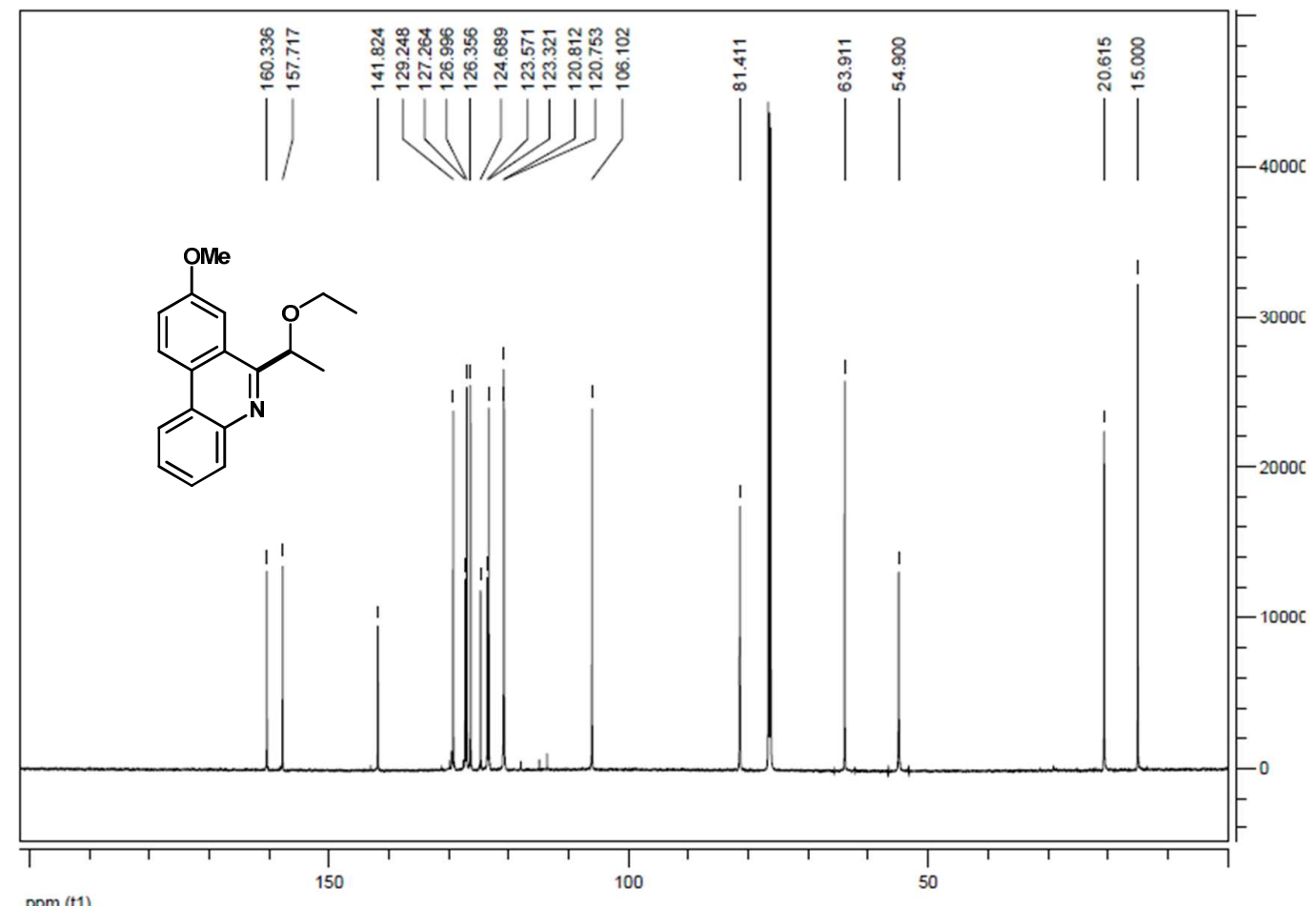

$\mathrm{ppm}(\mathrm{t} 1)$ 
6-(1-ethoxyethyl)-8-fluorophenanthridine (3i)

Proton NMR $\left(\mathrm{CDCl}_{3}\right)$

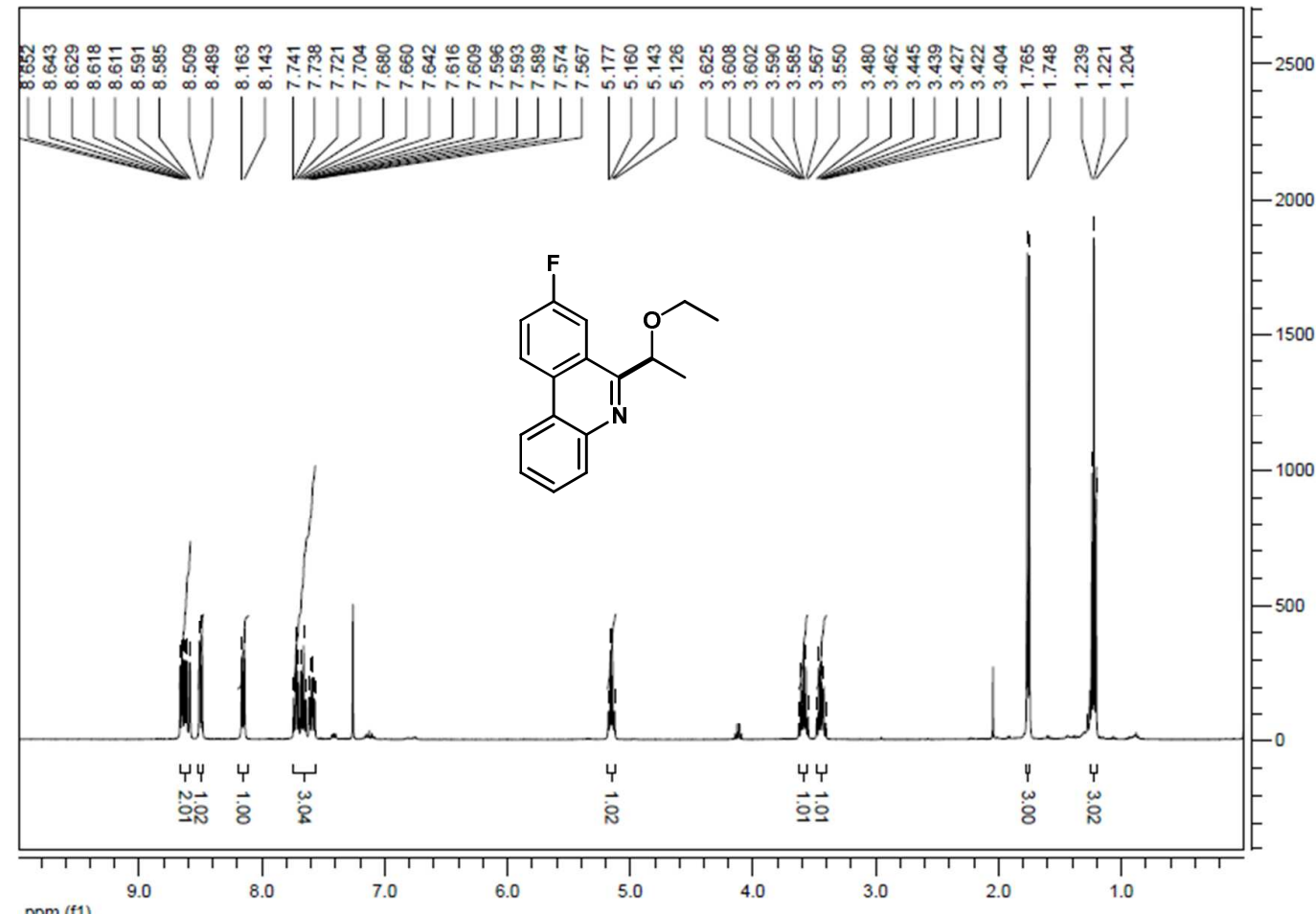

\section{Carbon NMR $\left(\mathrm{CDCl}_{3}\right)$}

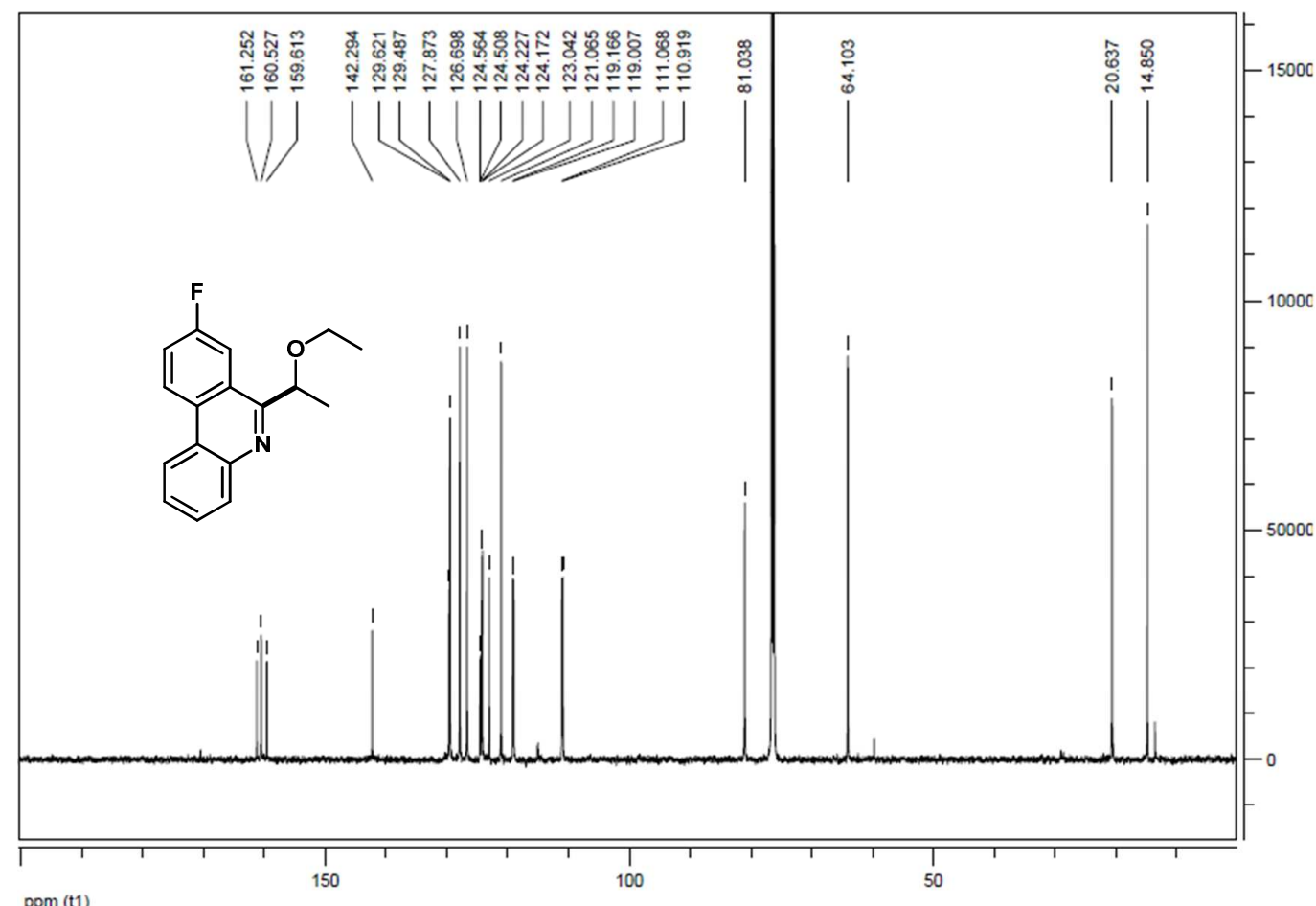


8-chloro-6-(1-ethoxyethyl)phenanthridine (3j)

Proton NMR $\left(\mathrm{CDCl}_{3}\right)$

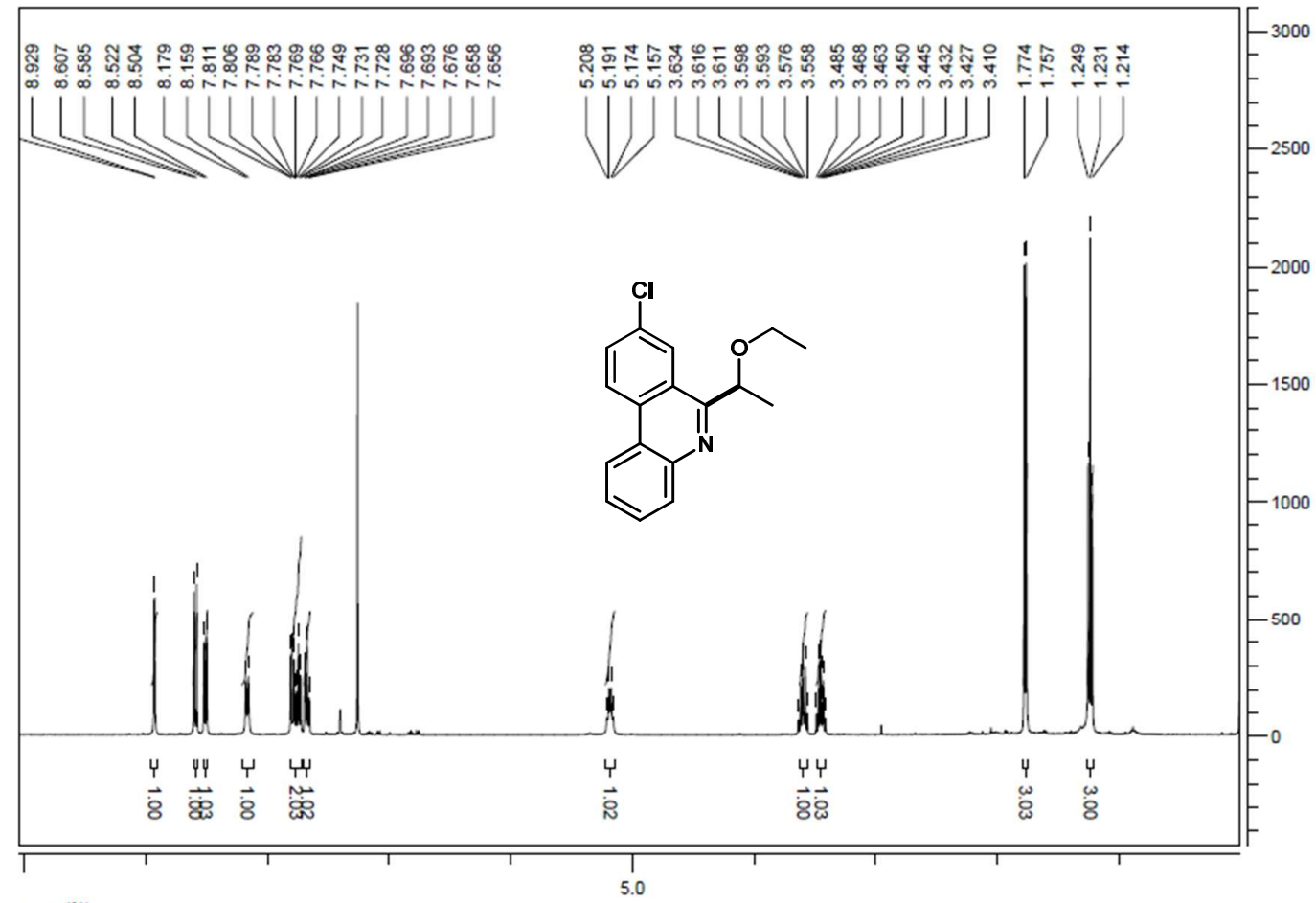

ppm (f1)

\section{Carbon NMR (CDCl $)_{3}$}

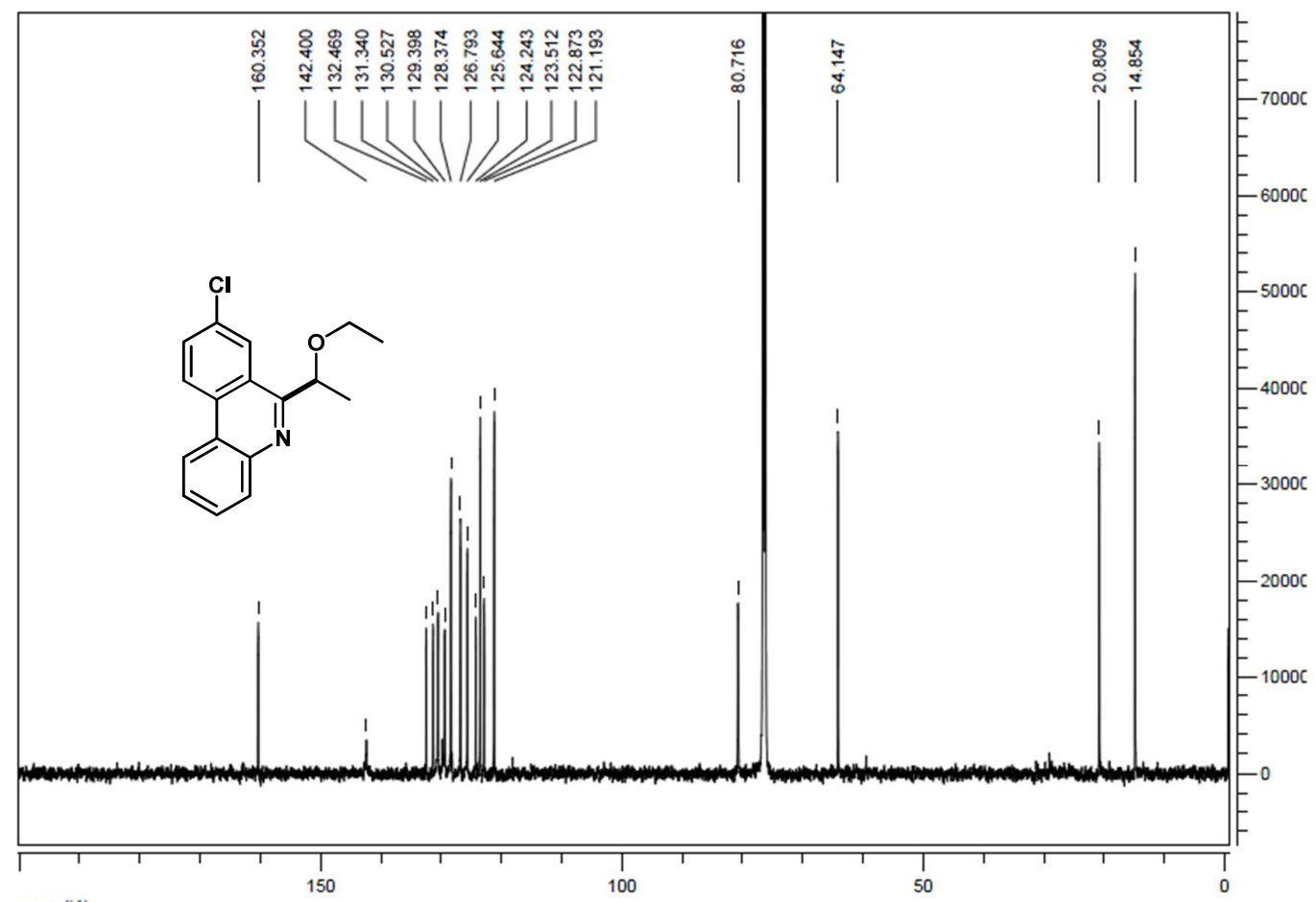

$\mathrm{ppm}(\mathrm{t} 1)$ 
6-(1-ethoxyethyl)-8-(trifluoromethyl)phenanthridine (3k)

Proton NMR $\left(\mathrm{CDCl}_{3}\right)$

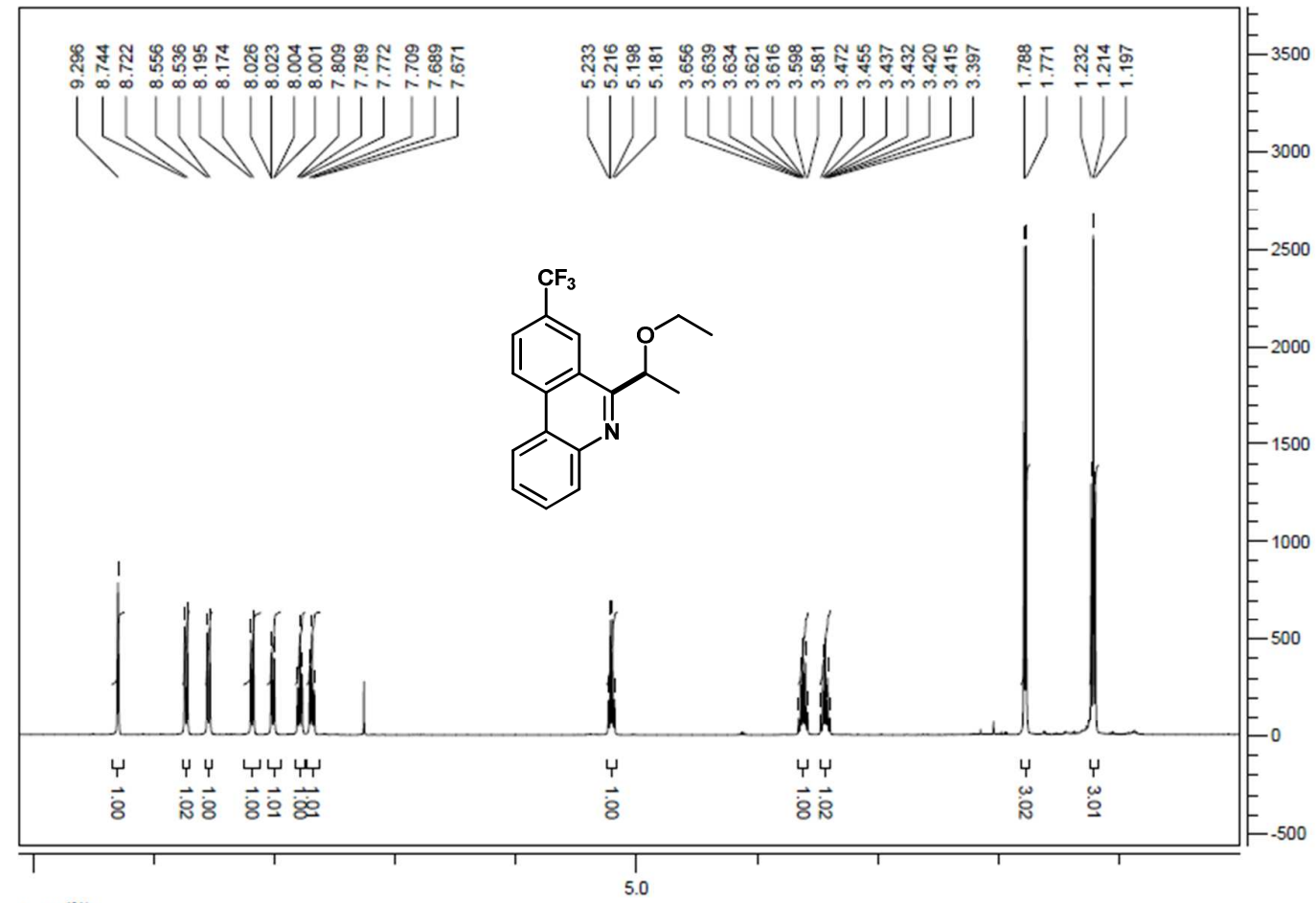

ppm (f1)

\section{Carbon NMR (CDCl $)_{3}$}

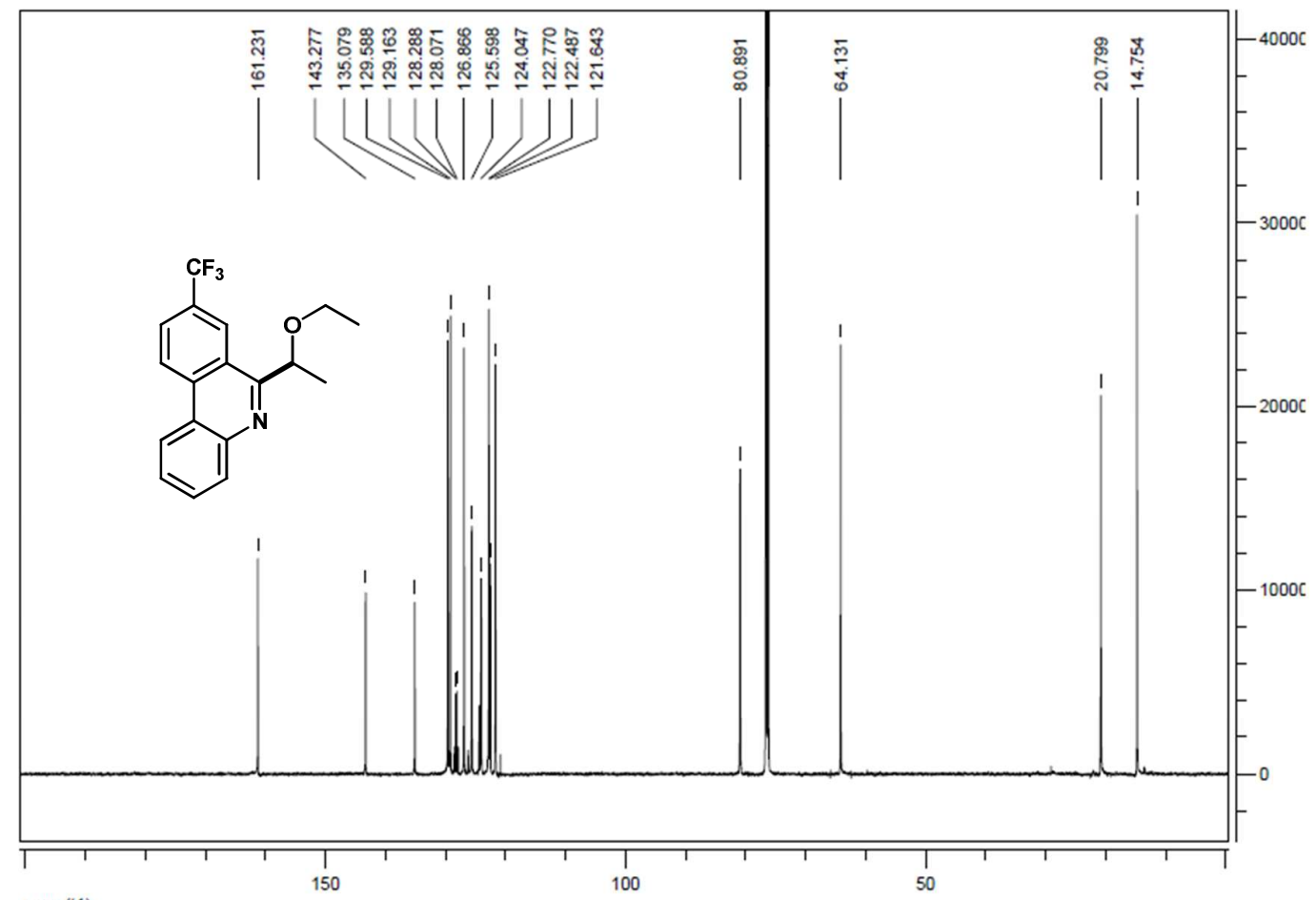

ppm (t1) 
6-(1-ethoxyethyl)-3-methylphenanthridine (3I)

Proton NMR $\left(\mathrm{CDCl}_{3}\right)$

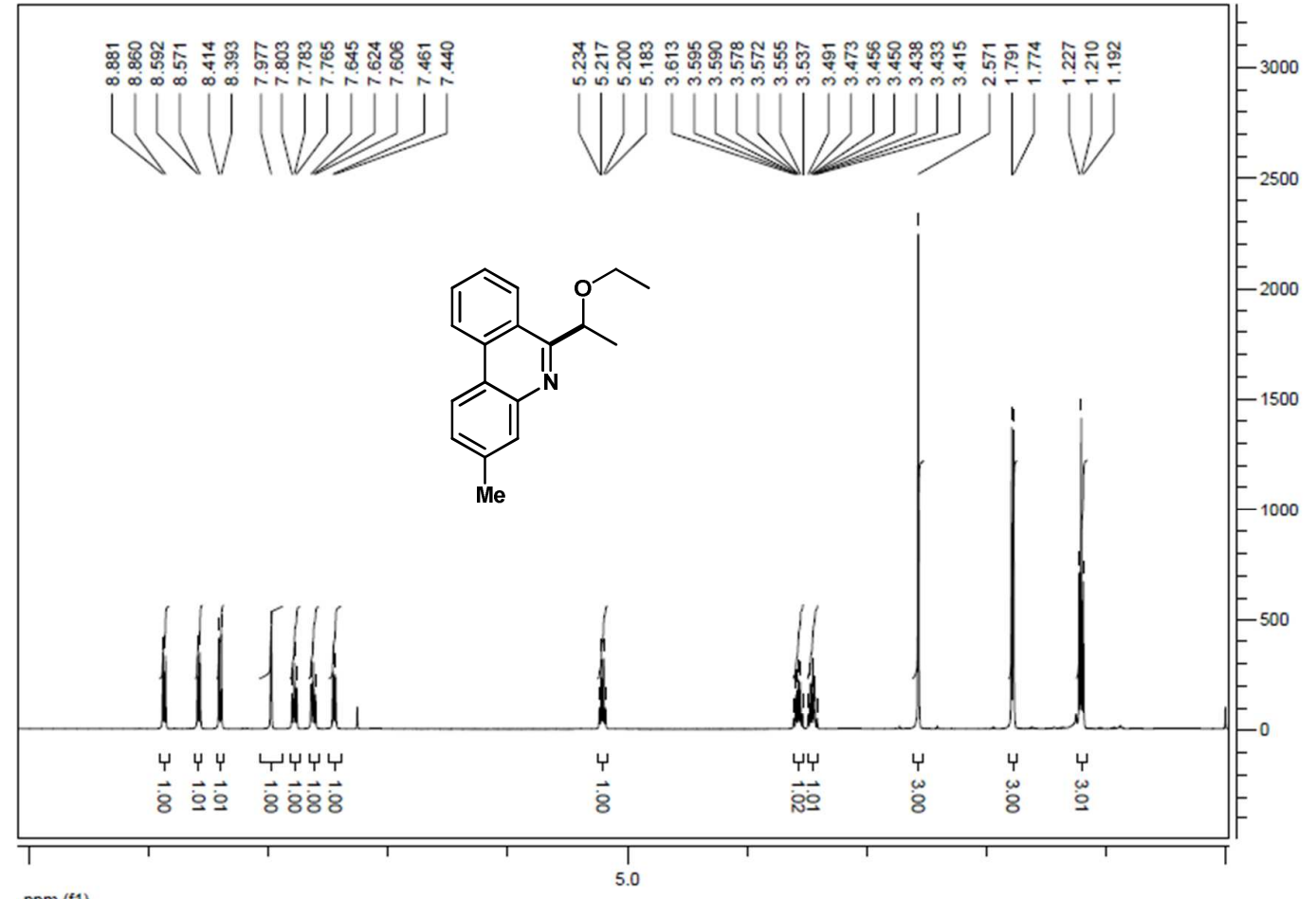

\section{Carbon NMR (CDCl $)_{3}$}

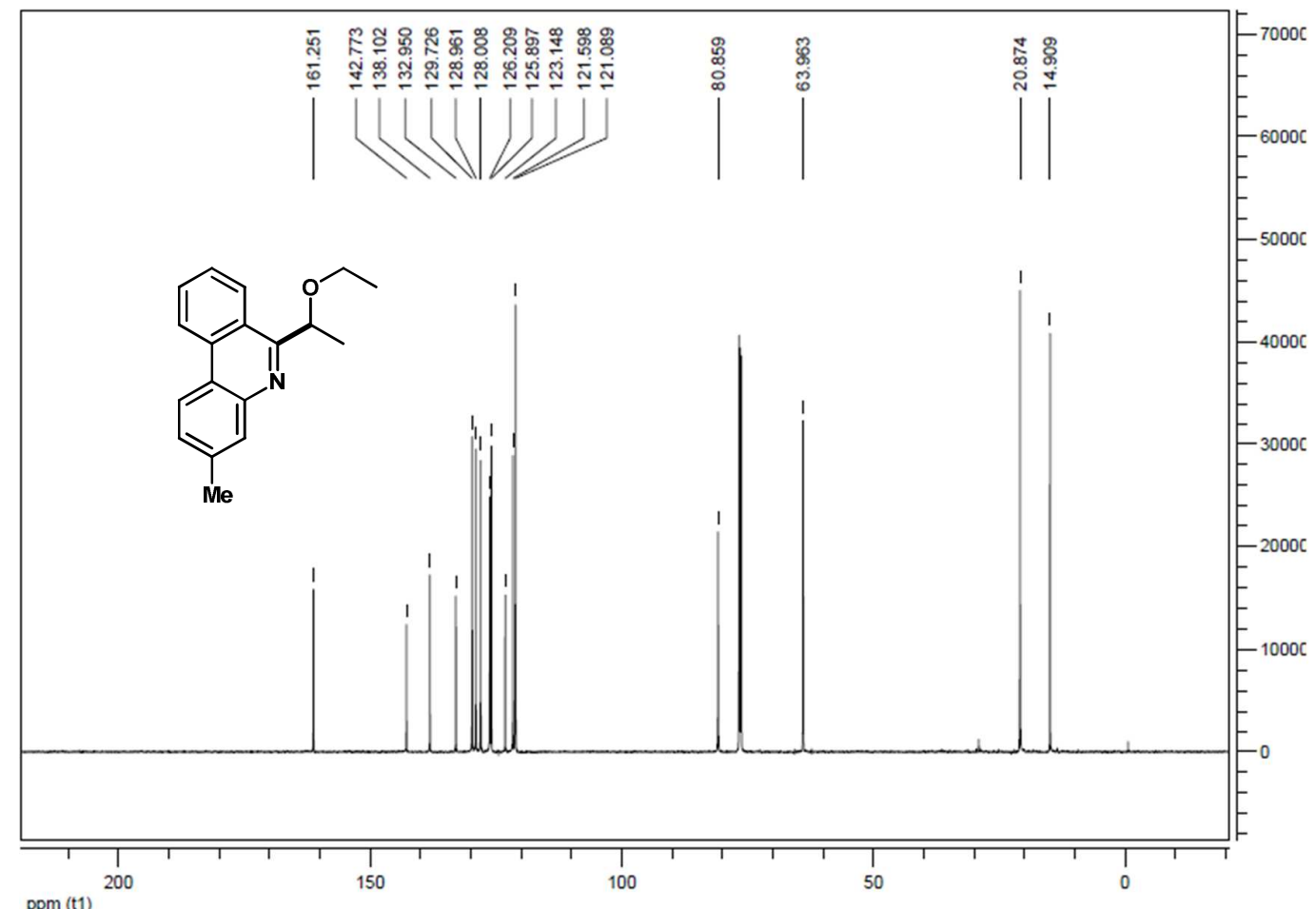


6-(1-ethoxyethyl)-3-methoxyphenanthridine (3m)

Proton NMR $\left(\mathrm{CDCl}_{3}\right)$

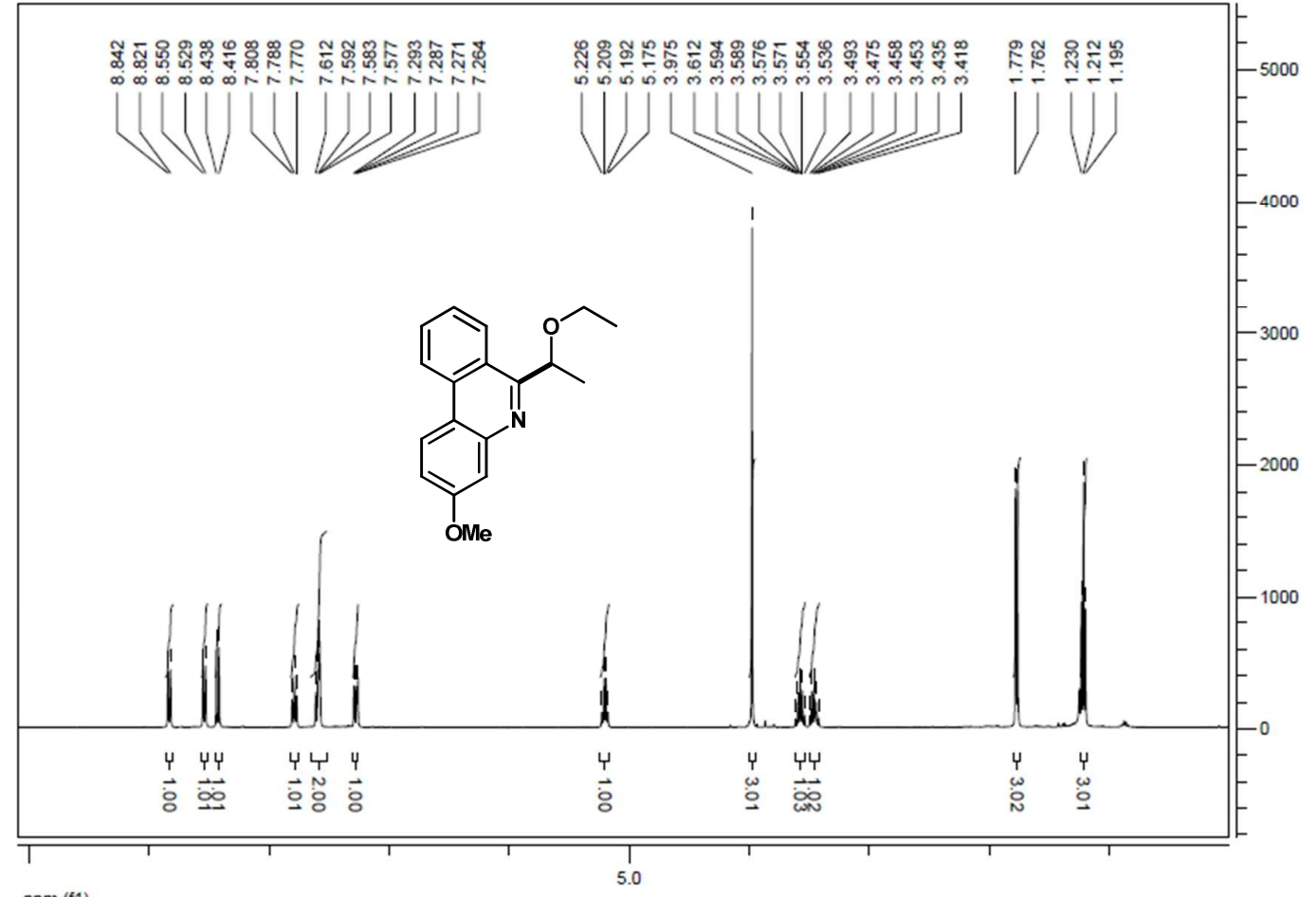

\section{Carbon NMR (CDCl $)_{3}$}

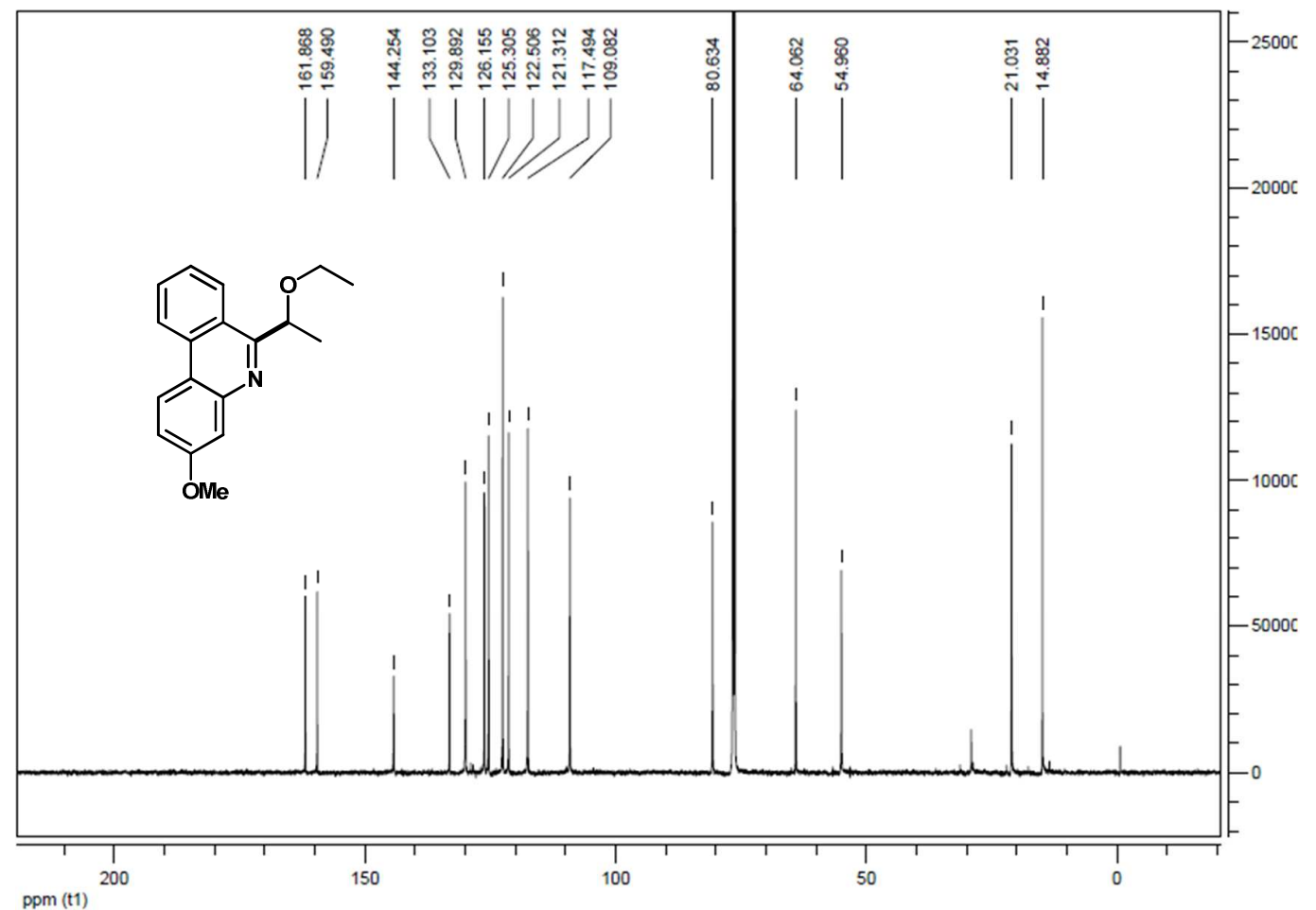


6-(1-ethoxyethyl)-9-methylphenanthridine (3n)

Proton NMR $\left(\mathrm{CDCl}_{3}\right)$

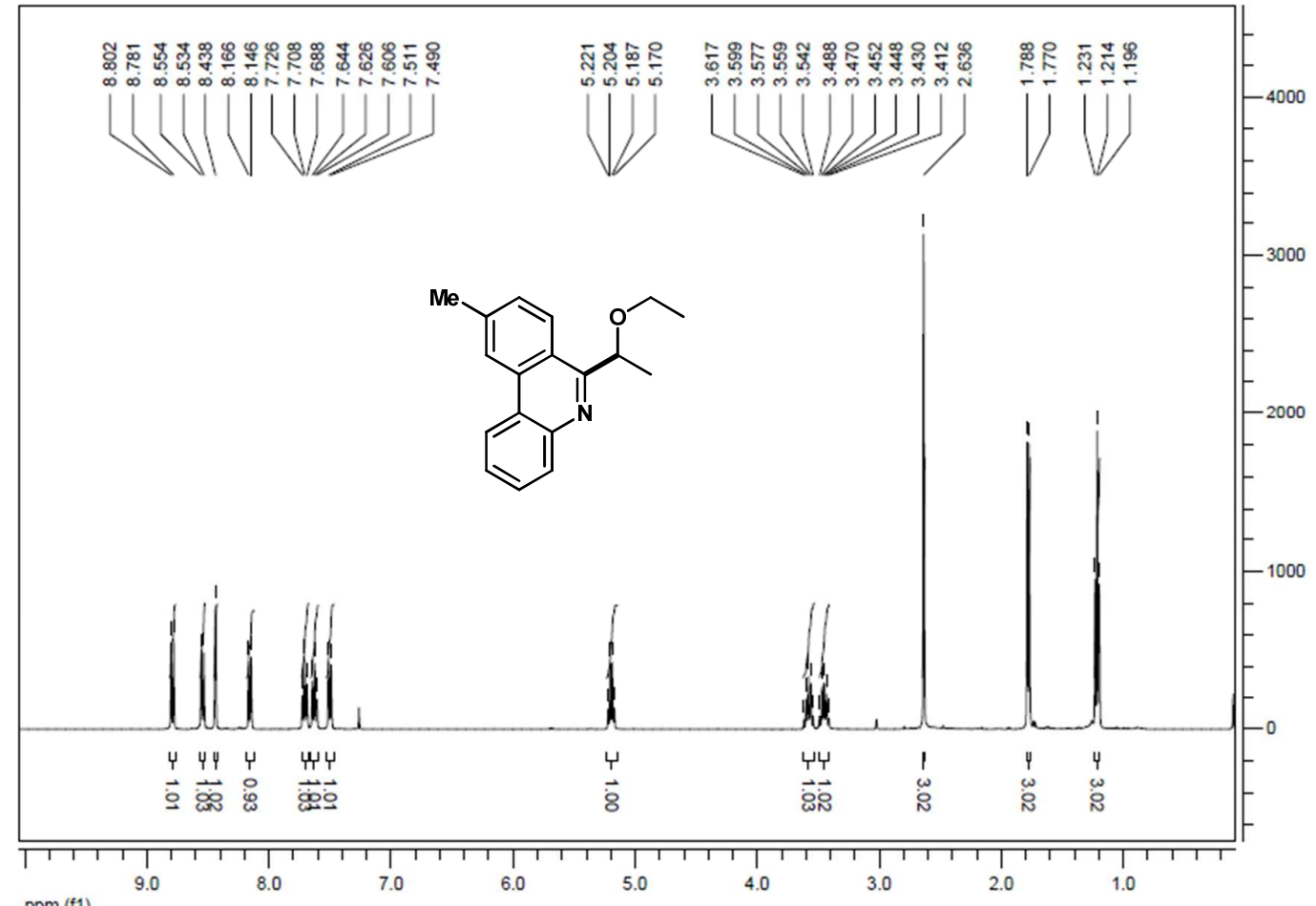

\section{Carbon NMR (CDCl $)_{3}$}

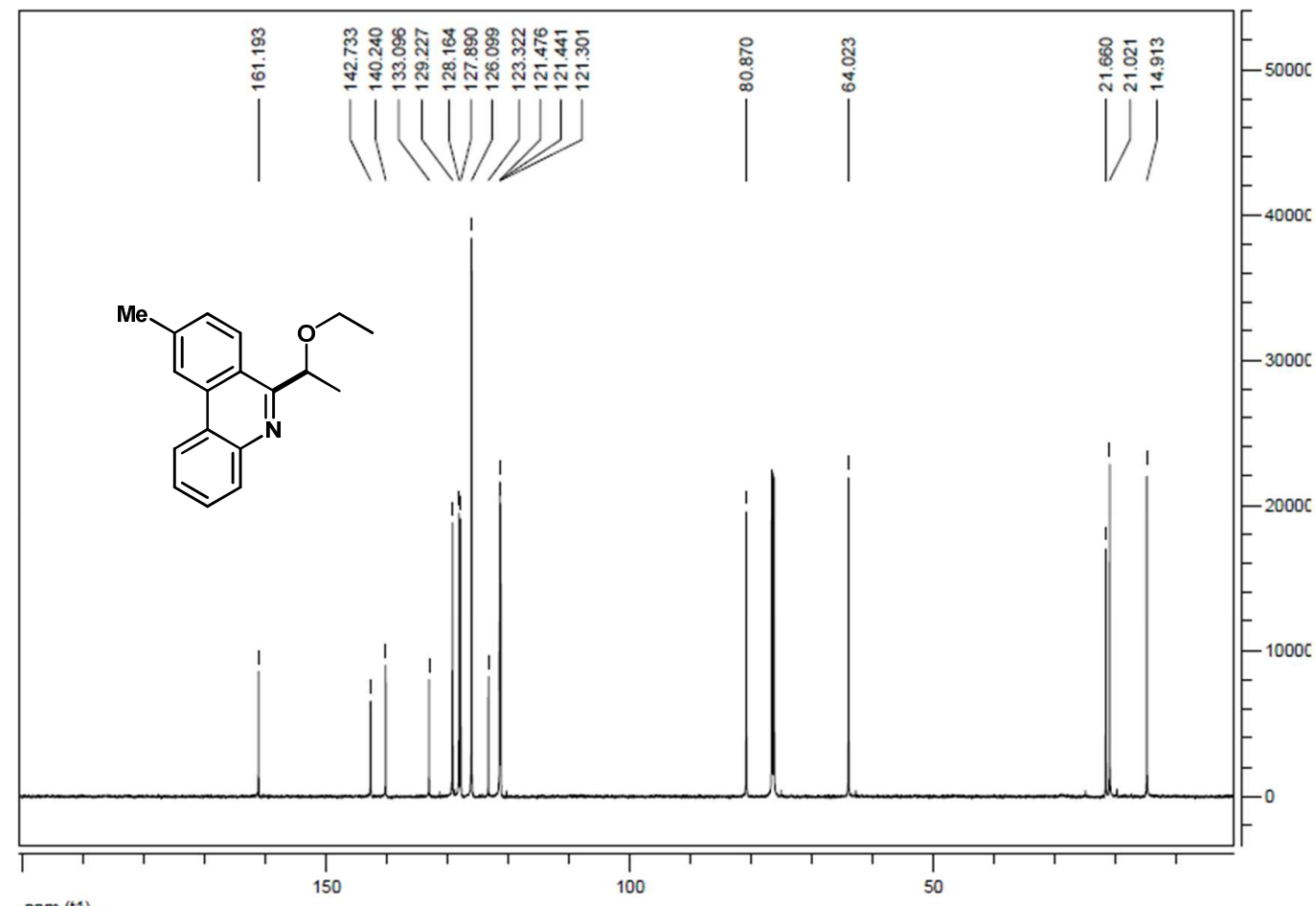

ppm (t1) 
6-(1-ethoxyethyl)-7-methylphenanthridine (3n')

Proton NMR $\left(\mathrm{CDCl}_{3}\right)$

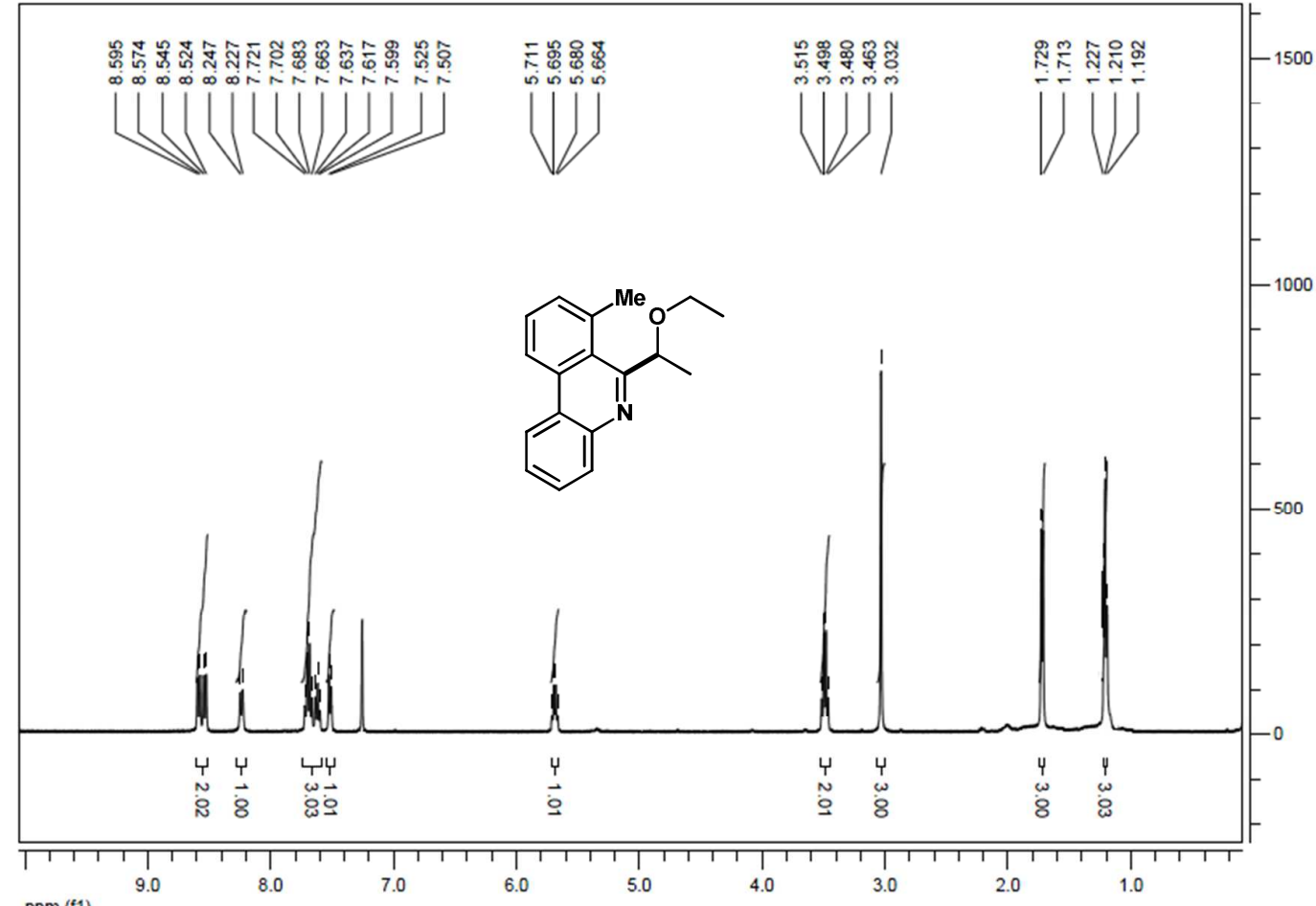

ppm (f1)

\section{Carbon NMR (CDCl $)$}

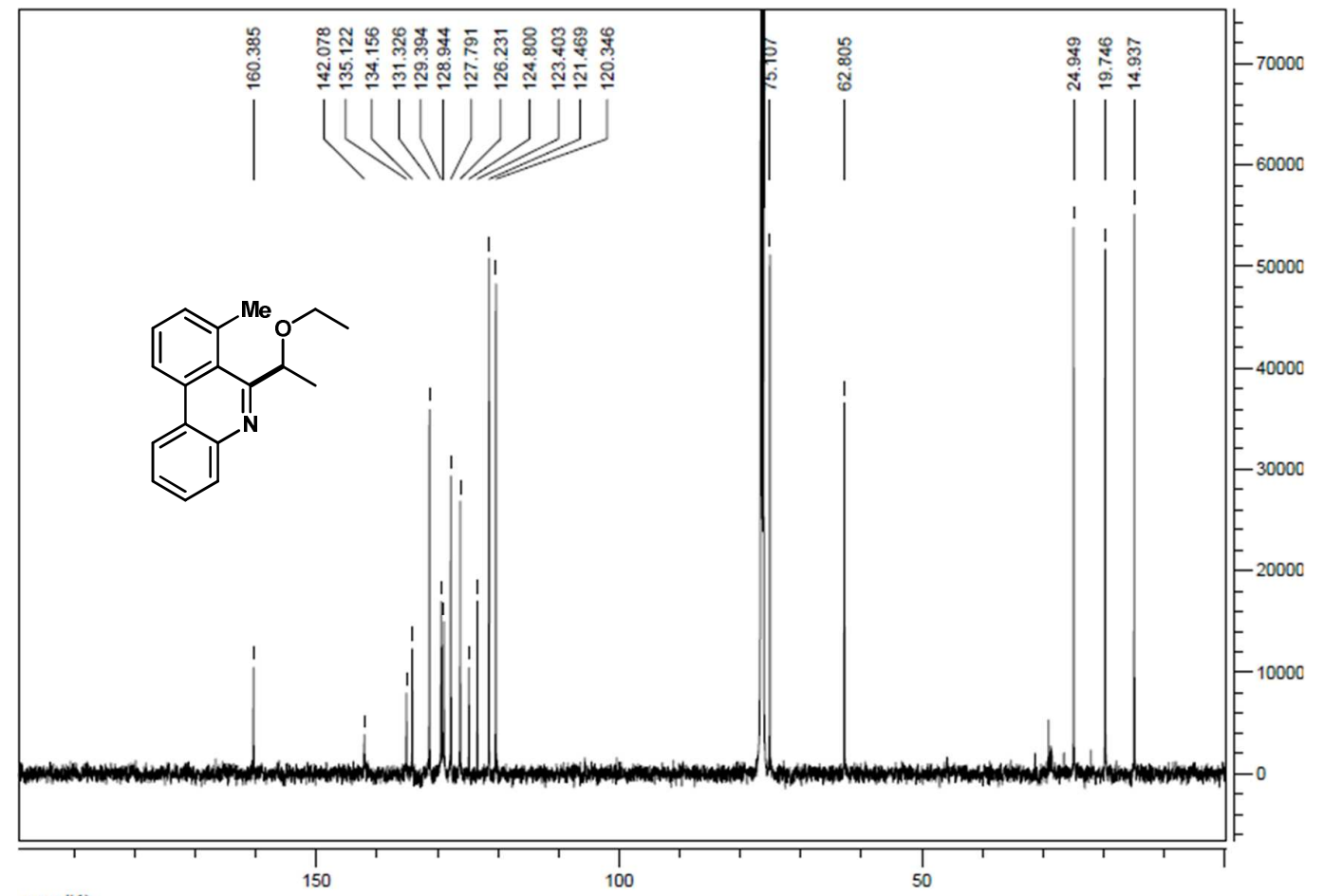

ppm (t1) 
6-(1-ethoxyethyl)-2,8-dimethylphenanthridine (3o)

Proton NMR $\left(\mathrm{CDCl}_{3}\right)$

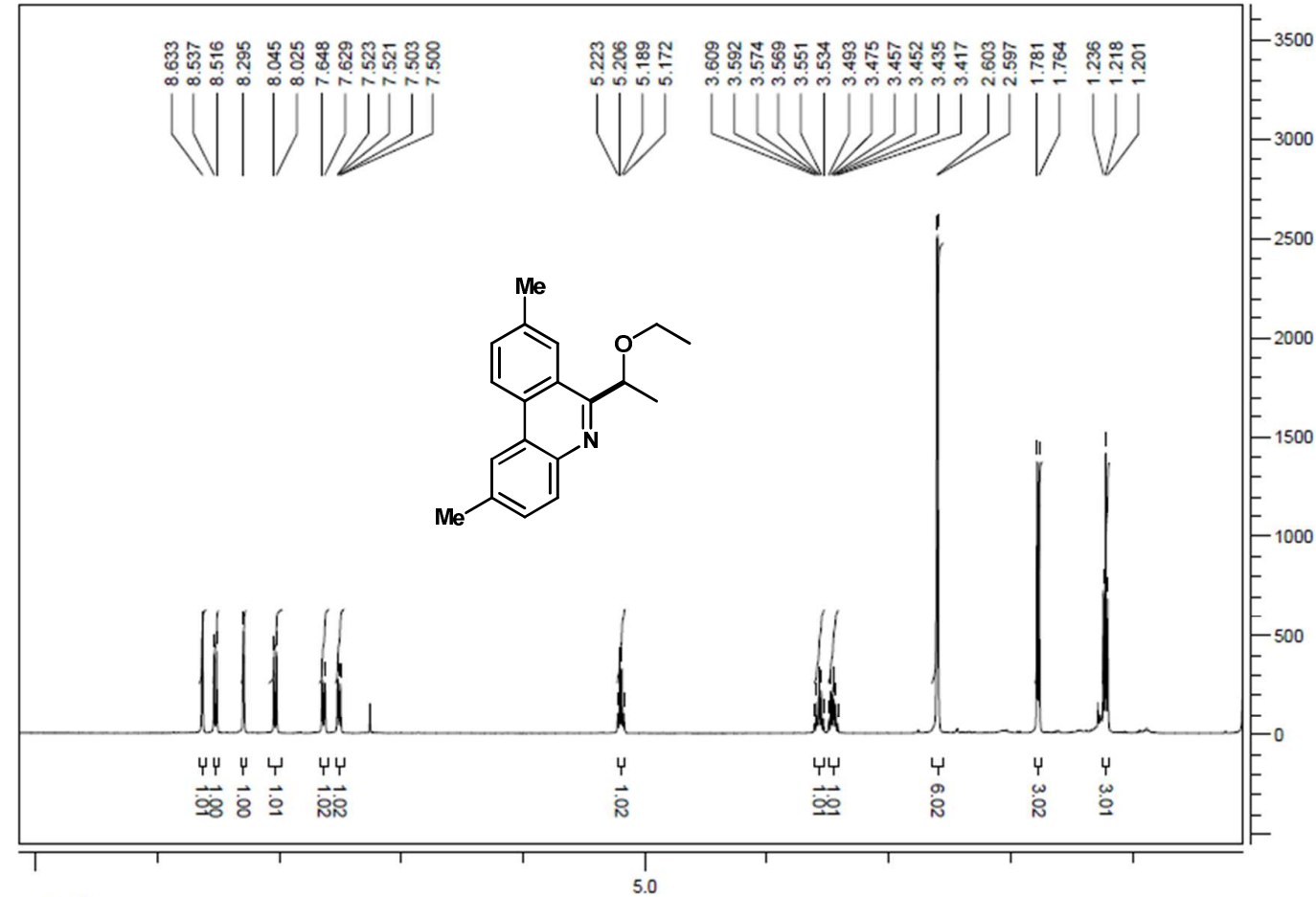

ppm (t1)

\section{Carbon NMR $\left(\mathrm{CDCl}_{3}\right)$}

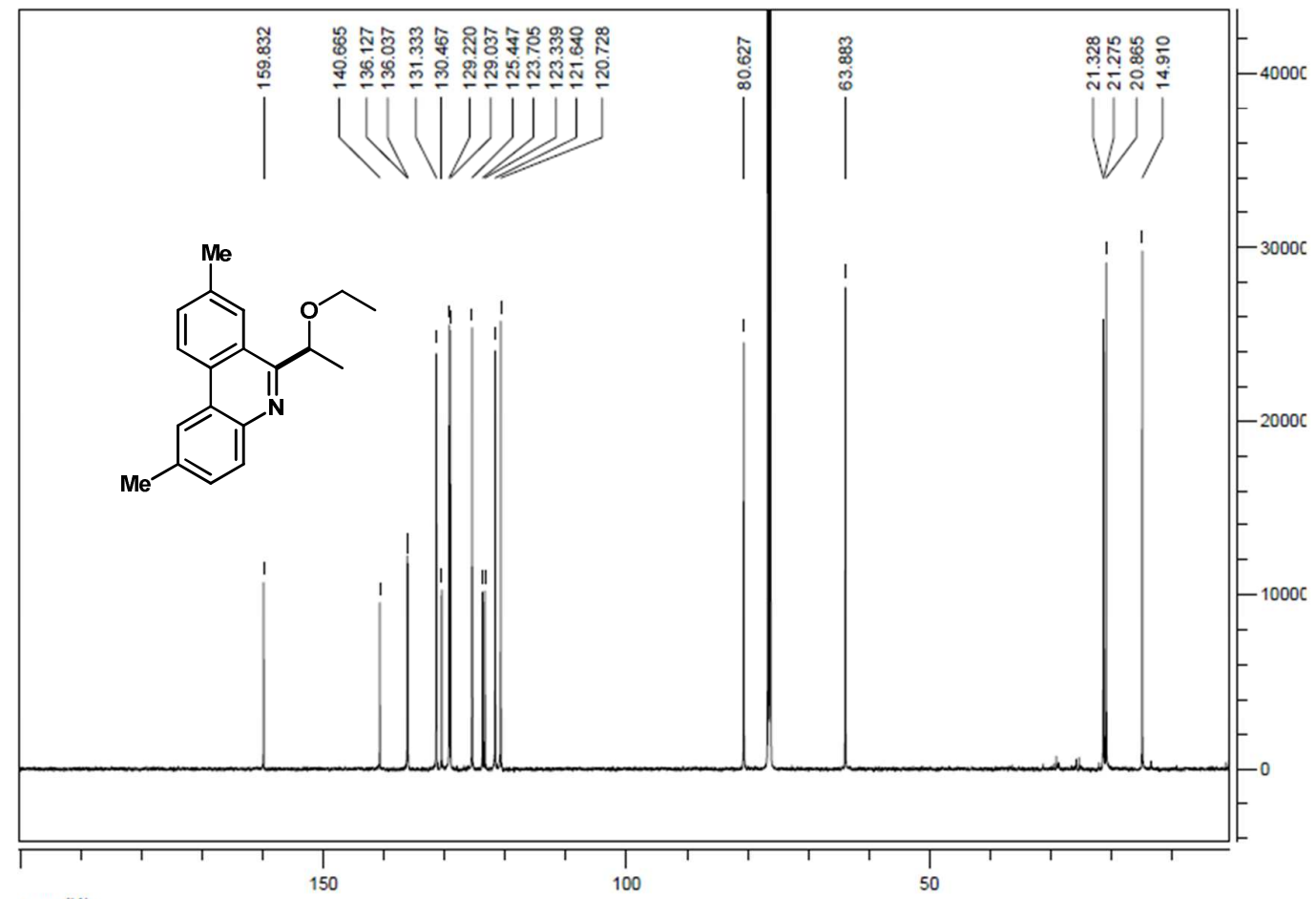

ppm (t1) 
6-(1-ethoxyethyl)-8-fluoro-2-methylphenanthridine (3p)

Proton NMR $\left(\mathrm{CDCl}_{3}\right)$

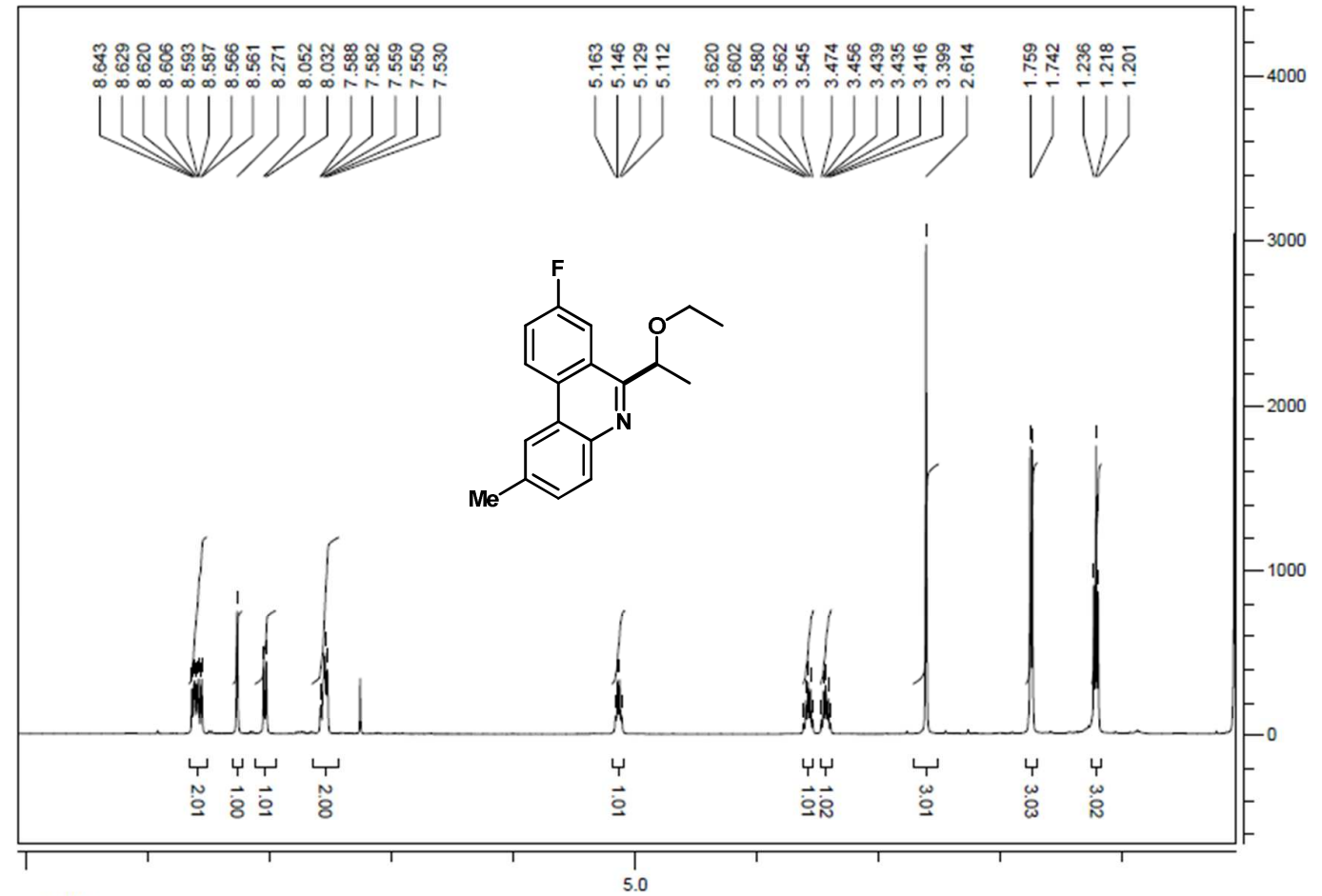

ppm (f1)

\section{Carbon NMR (CDCl $)_{3}$}

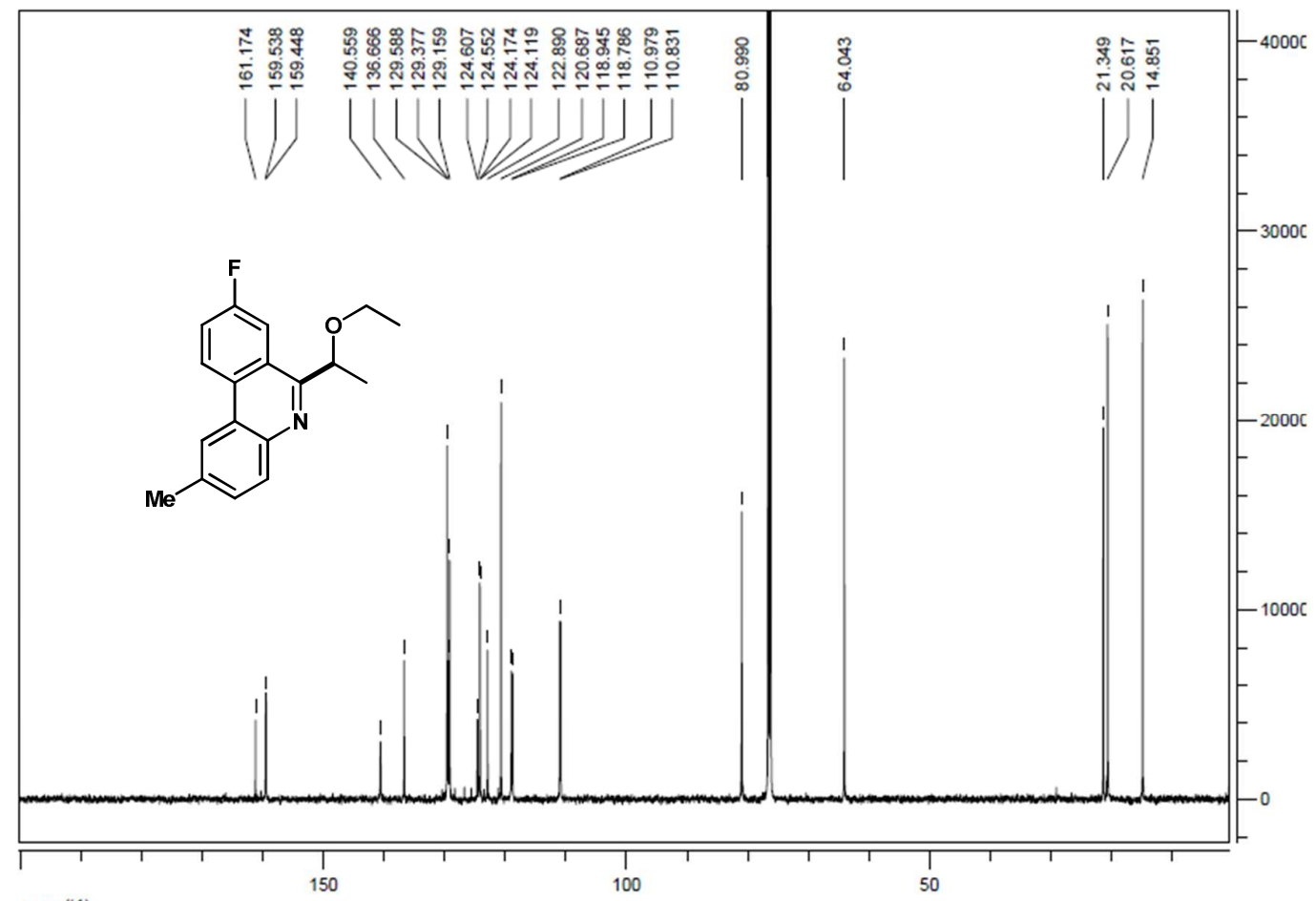

ppm (t1) 
6-(1-ethoxyethyl)-2-methyl-[1,3]dioxolo[4,5-j]phenanthridine (3q)

Proton NMR $\left(\mathrm{CDCl}_{3}\right)$

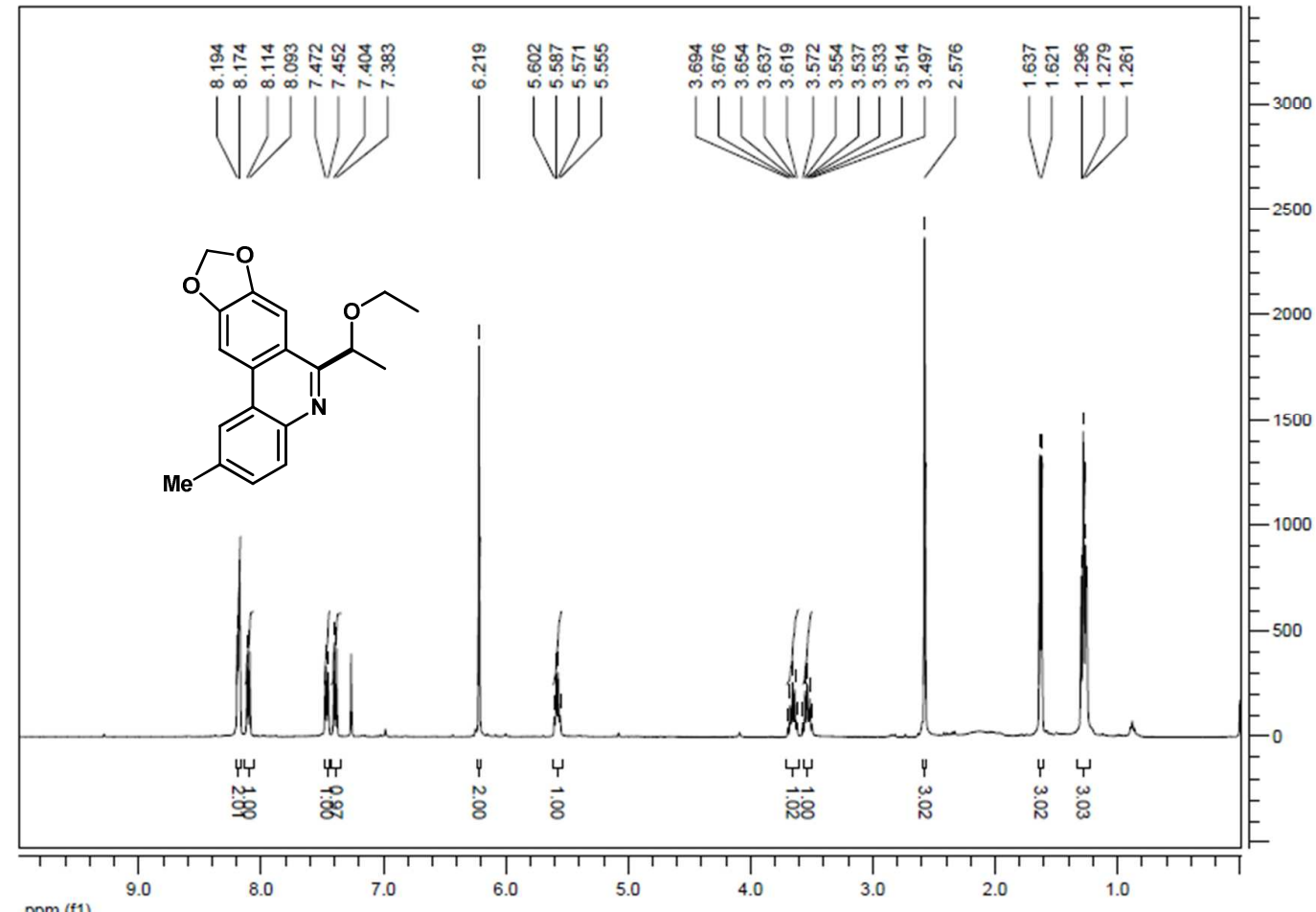

Carbon NMR (CDCl $\left.)_{3}\right)$

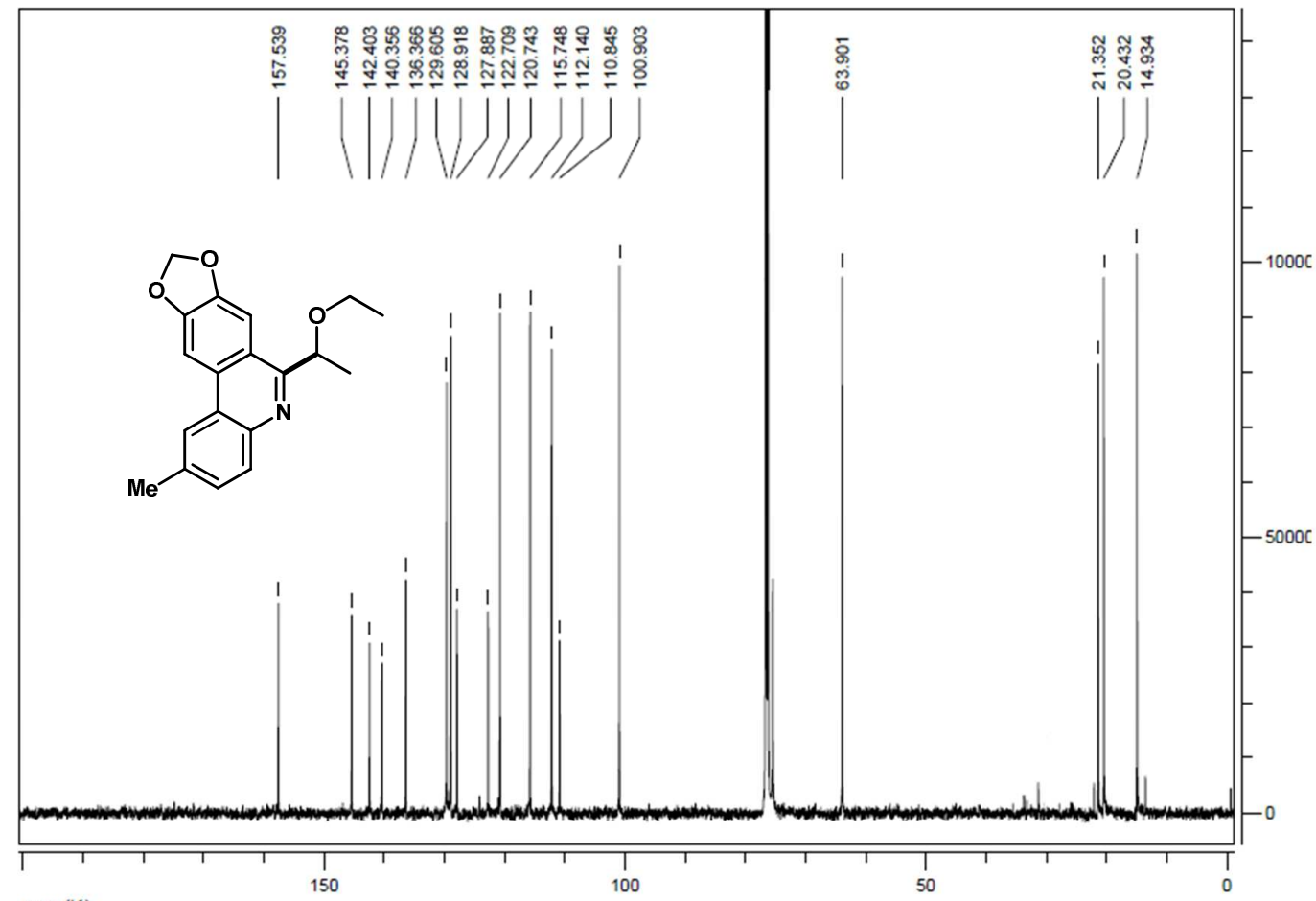

ppm (t1) 


\section{4-(1-ethoxyethyl)-8-methyl-[1,3] dioxolo[4,5-i]phenanthridine (3q')}

Proton NMR $\left(\mathrm{CDCl}_{3}\right)$

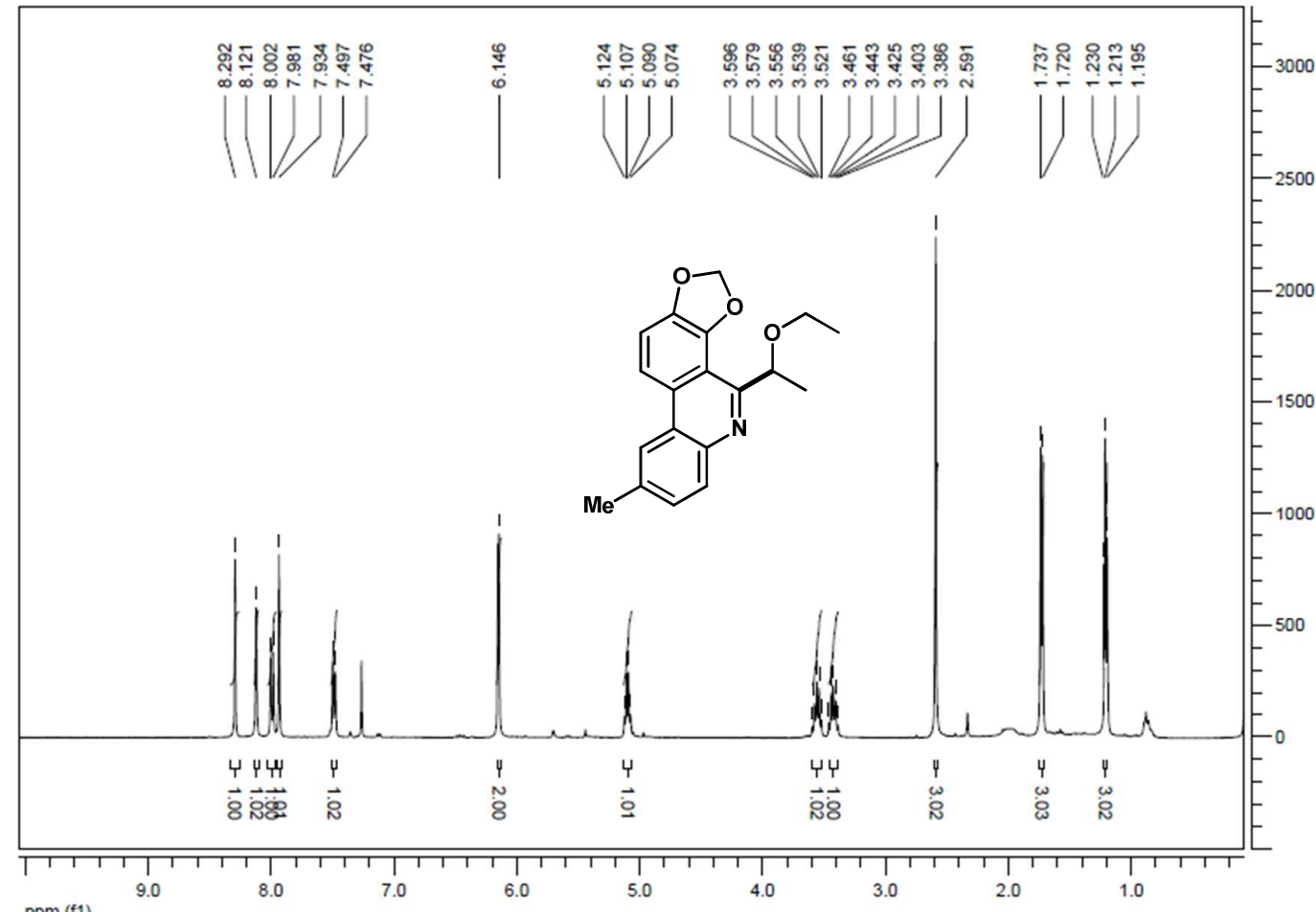

\section{Carbon NMR $\left(\mathrm{CDCl}_{3}\right)$}

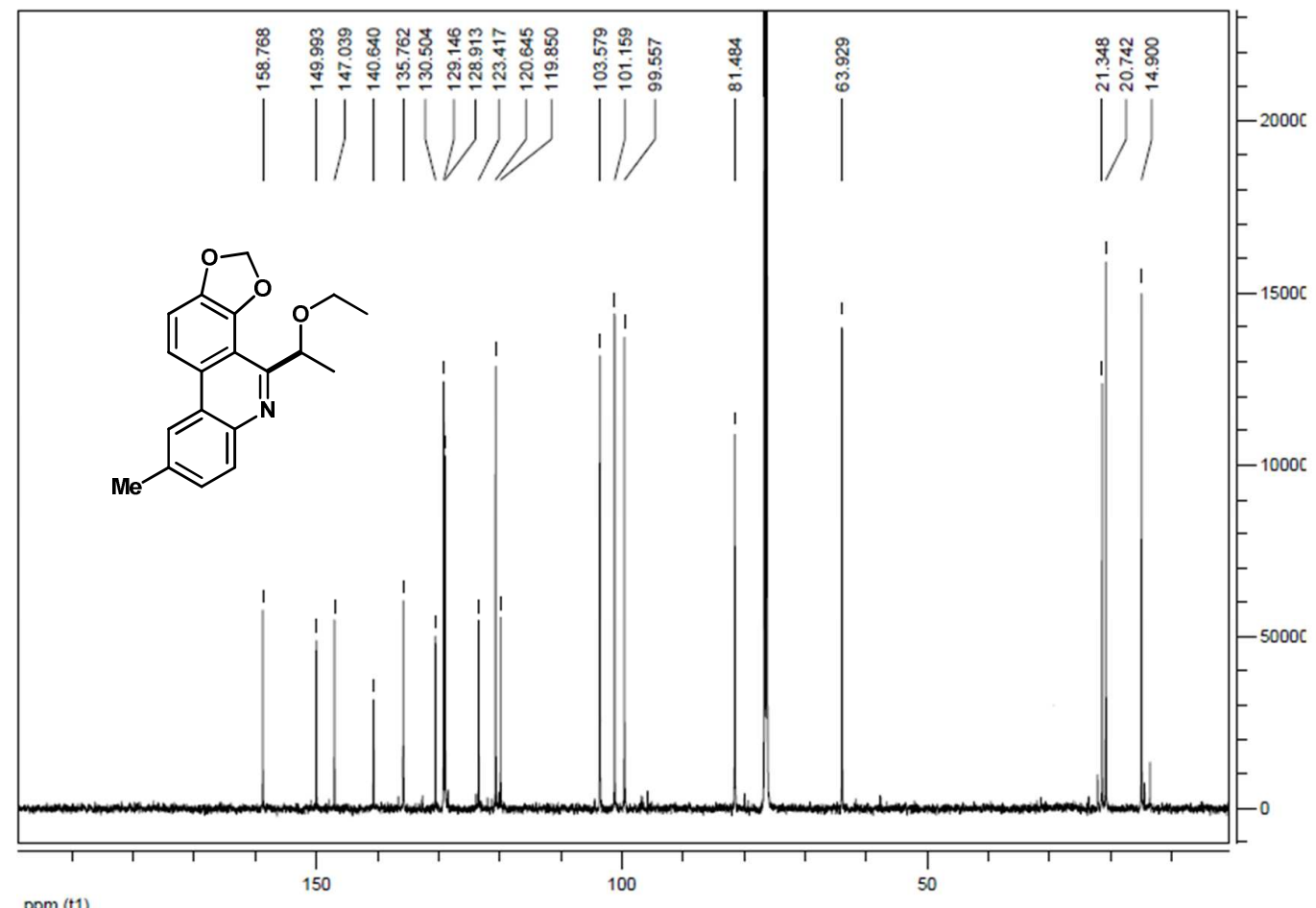


6-(1,2-dimethoxyethyl)phenanthridine (4b)

Proton NMR $\left(\mathrm{CDCl}_{3}\right)$

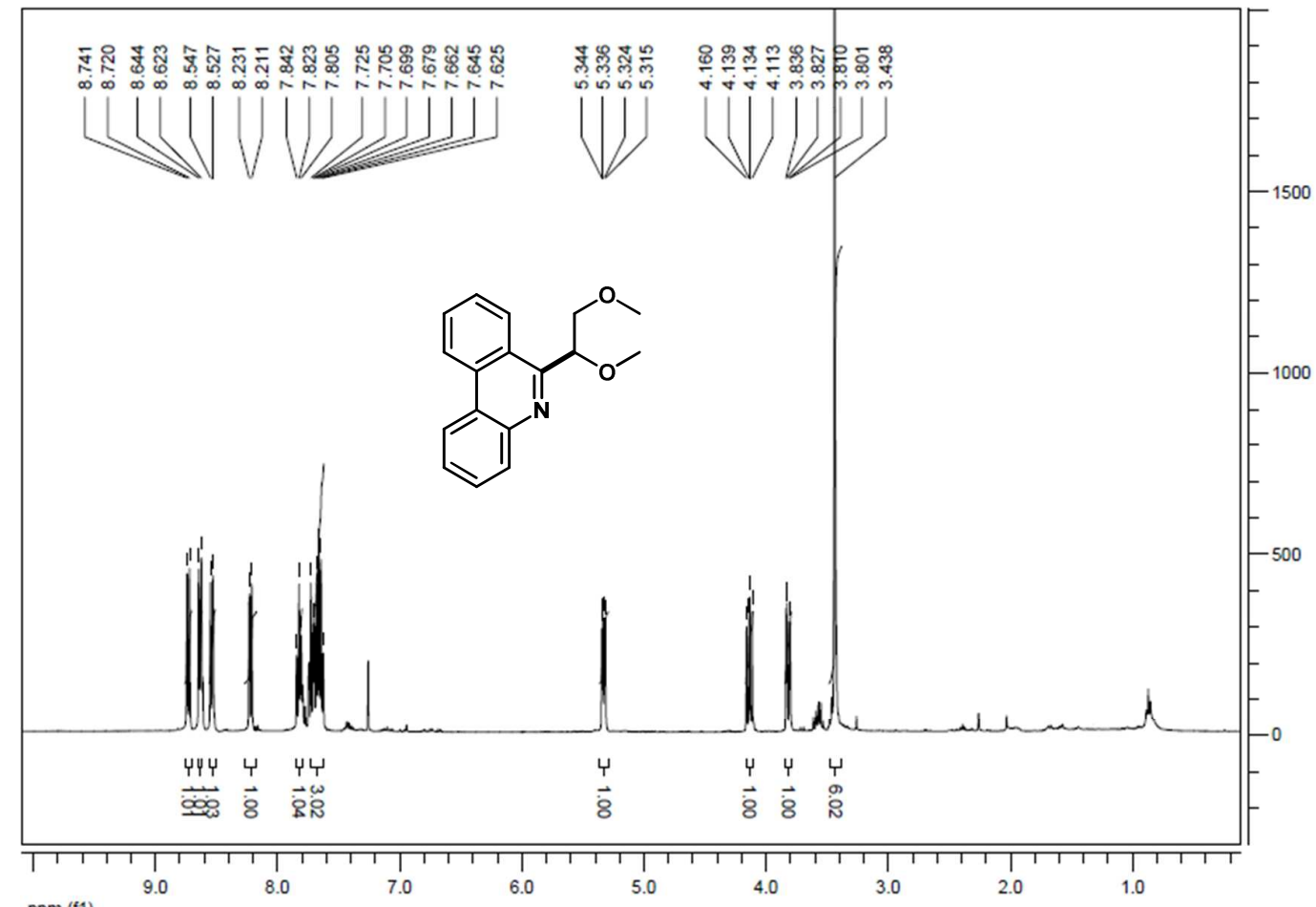

ppm (f1)

\section{Carbon NMR $\left(\mathrm{CDCl}_{3}\right)$}

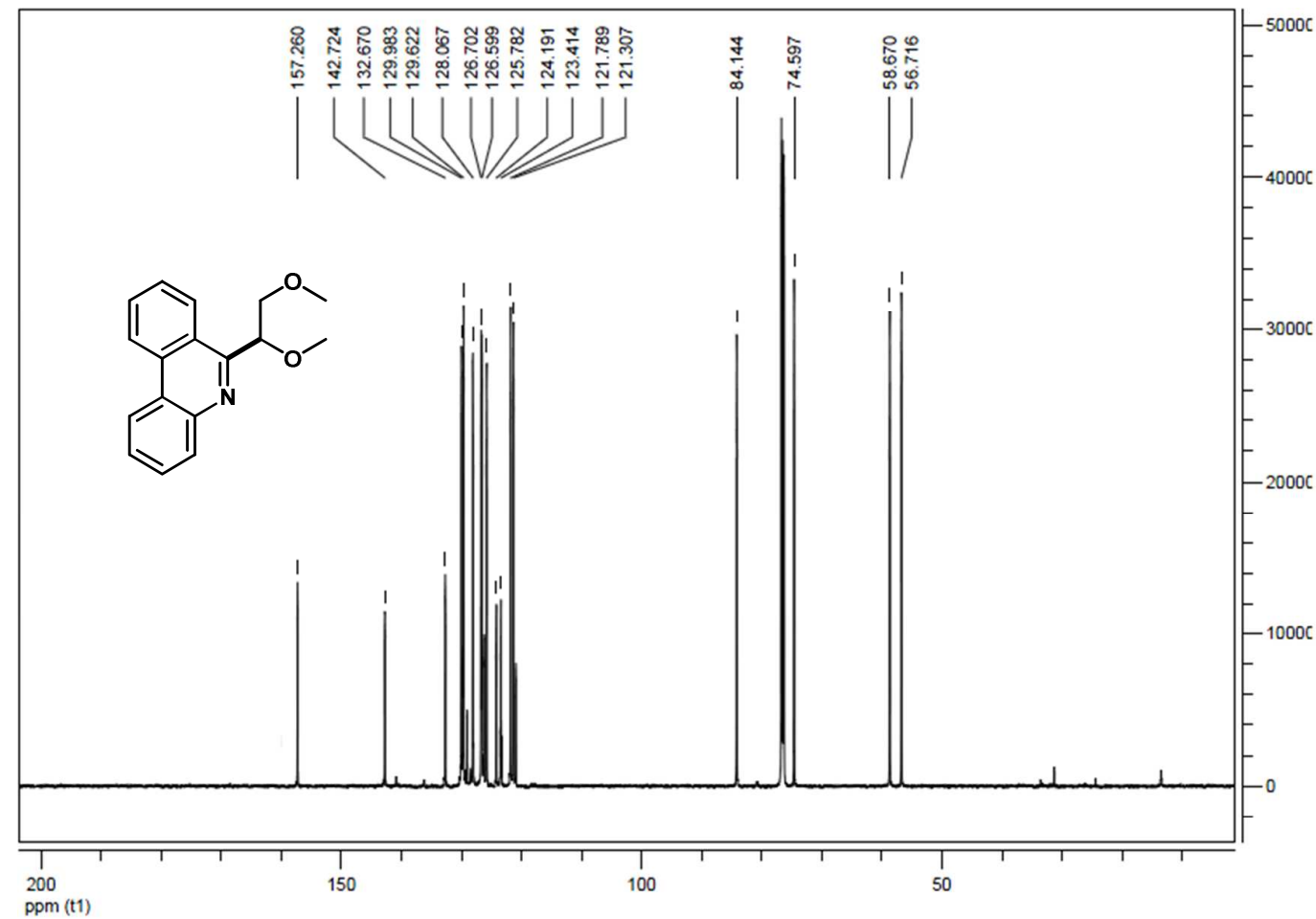


6-(tetrahydrofuran-2-yl)phenanthridine (4c)

Proton NMR $\left(\mathrm{CDCl}_{3}\right)$

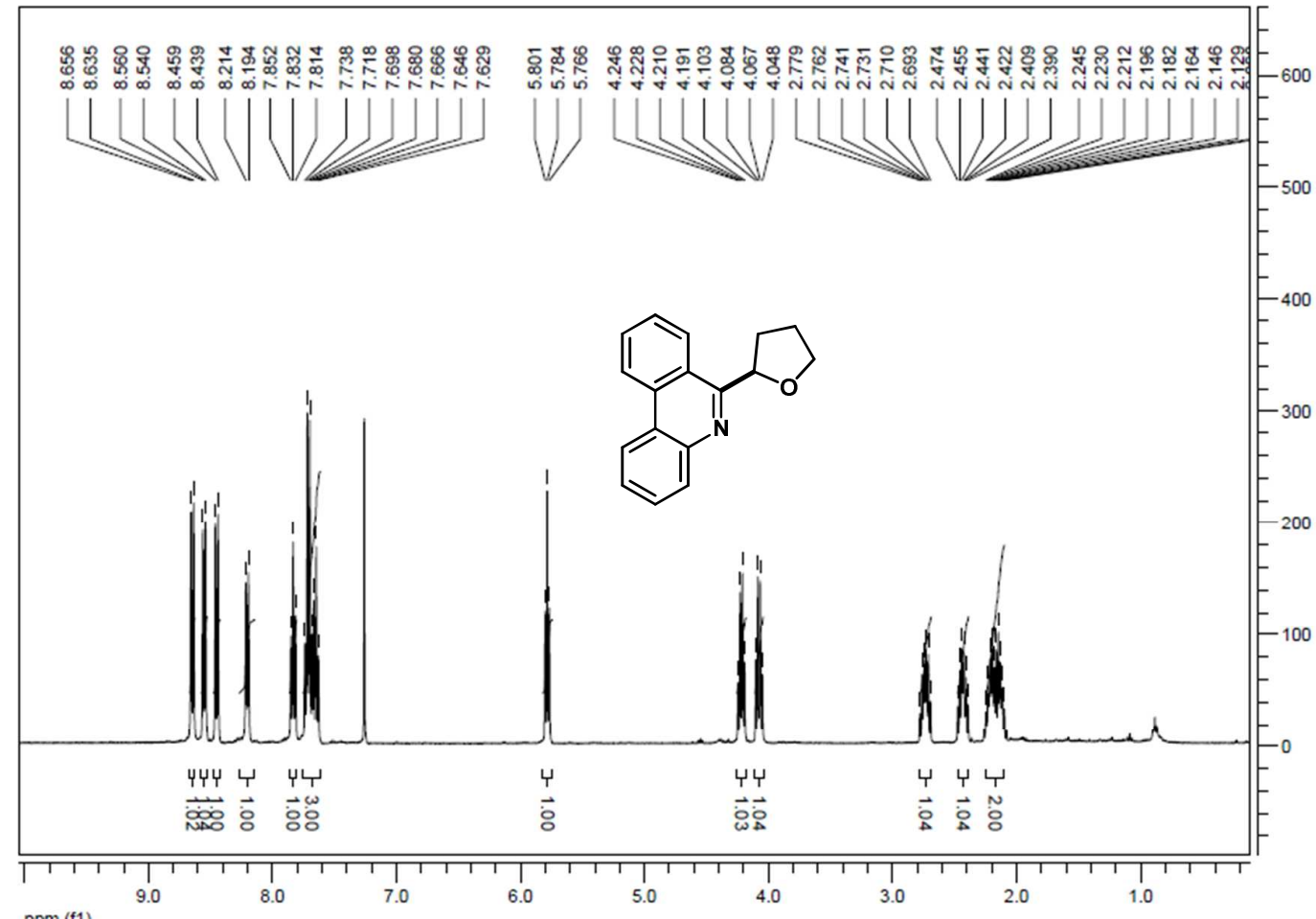

ppm (f1)

\section{Carbon NMR (CDCl $)_{3}$}

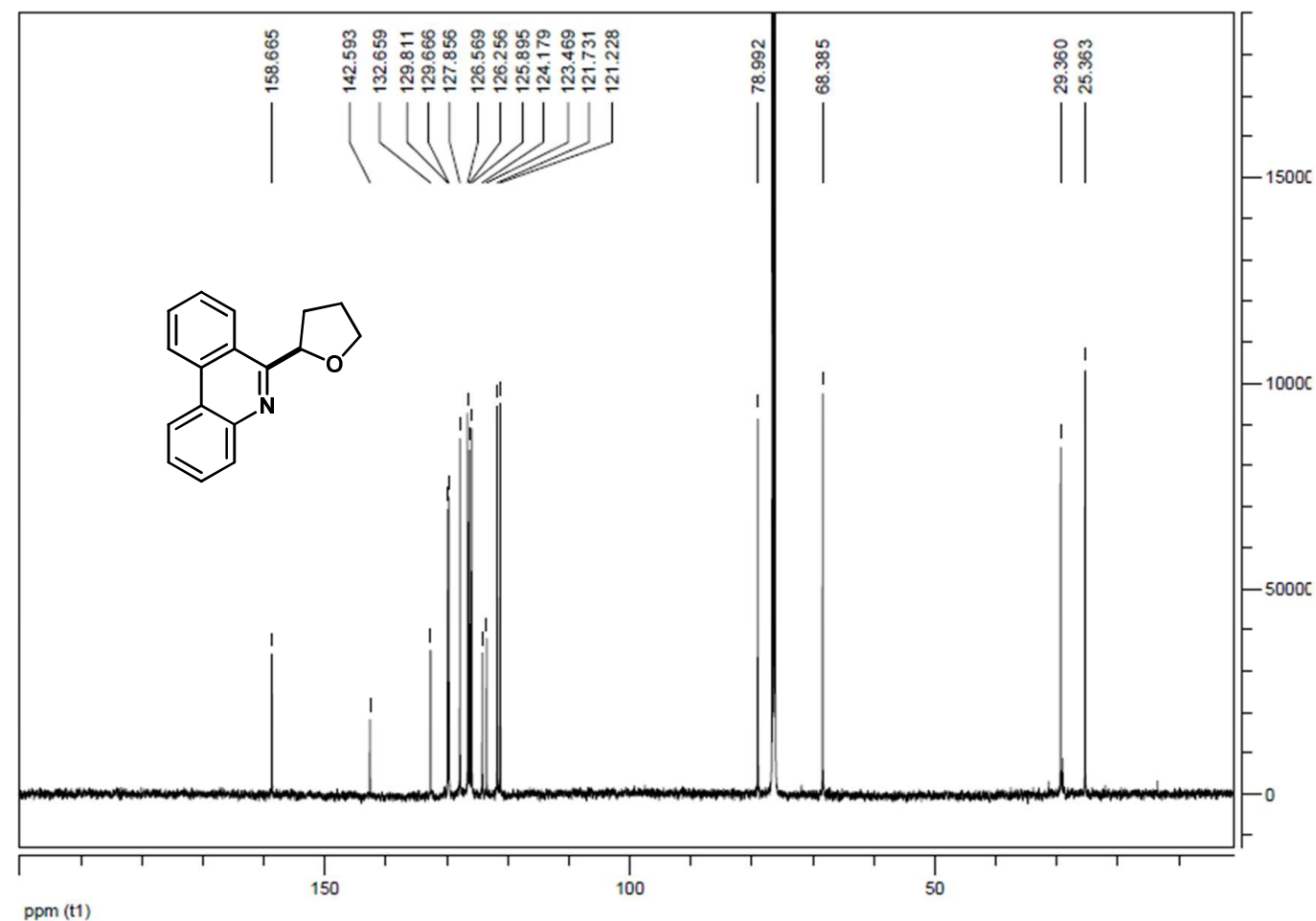

$\mathrm{ppm}(\mathrm{t} 1)$ 
6-(1,4-dioxan-2-yl)phenanthridine (4d)

Proton NMR $\left(\mathrm{CDCl}_{3}\right)$

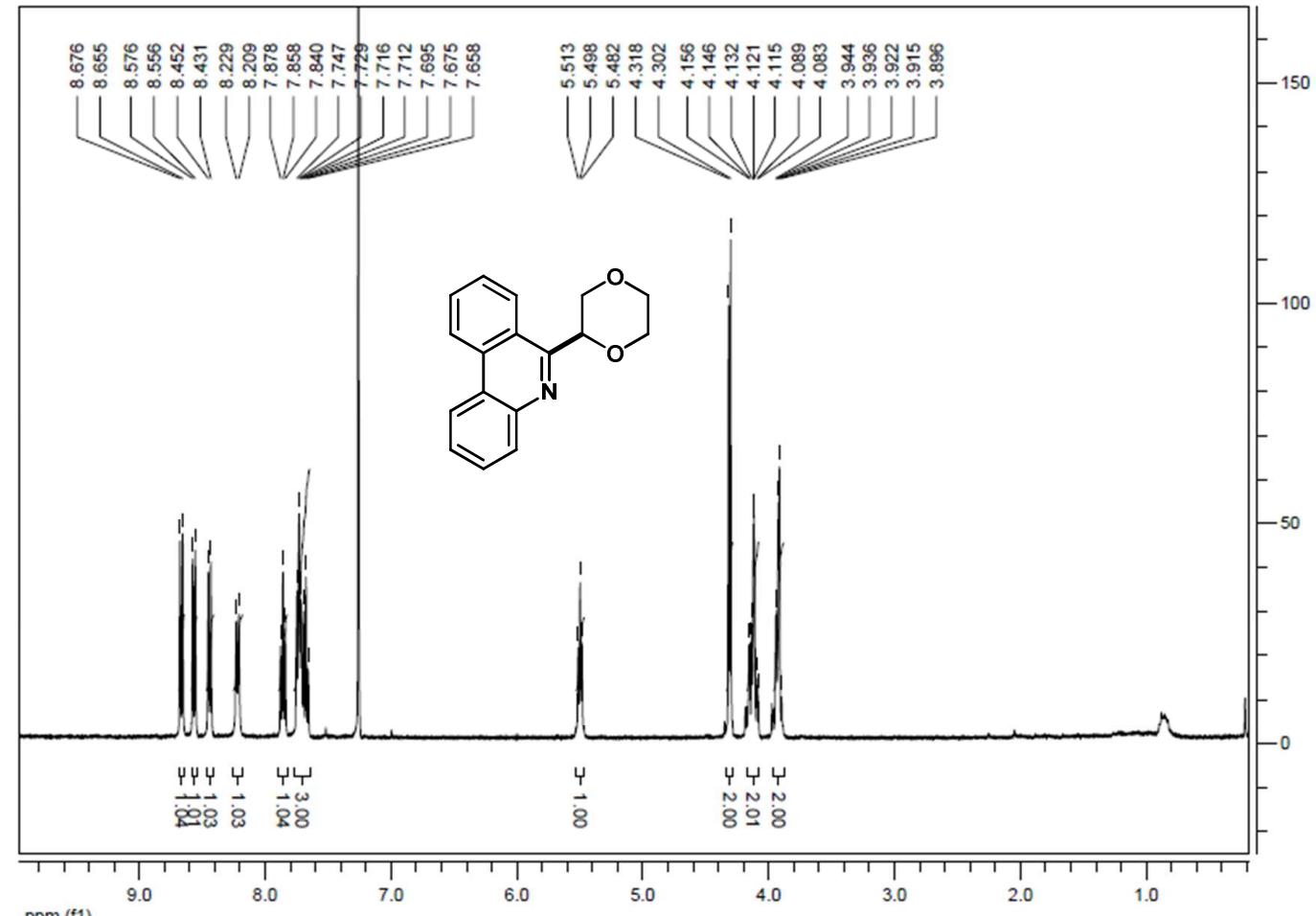

Carbon NMR (CDCl $\left.)_{3}\right)$

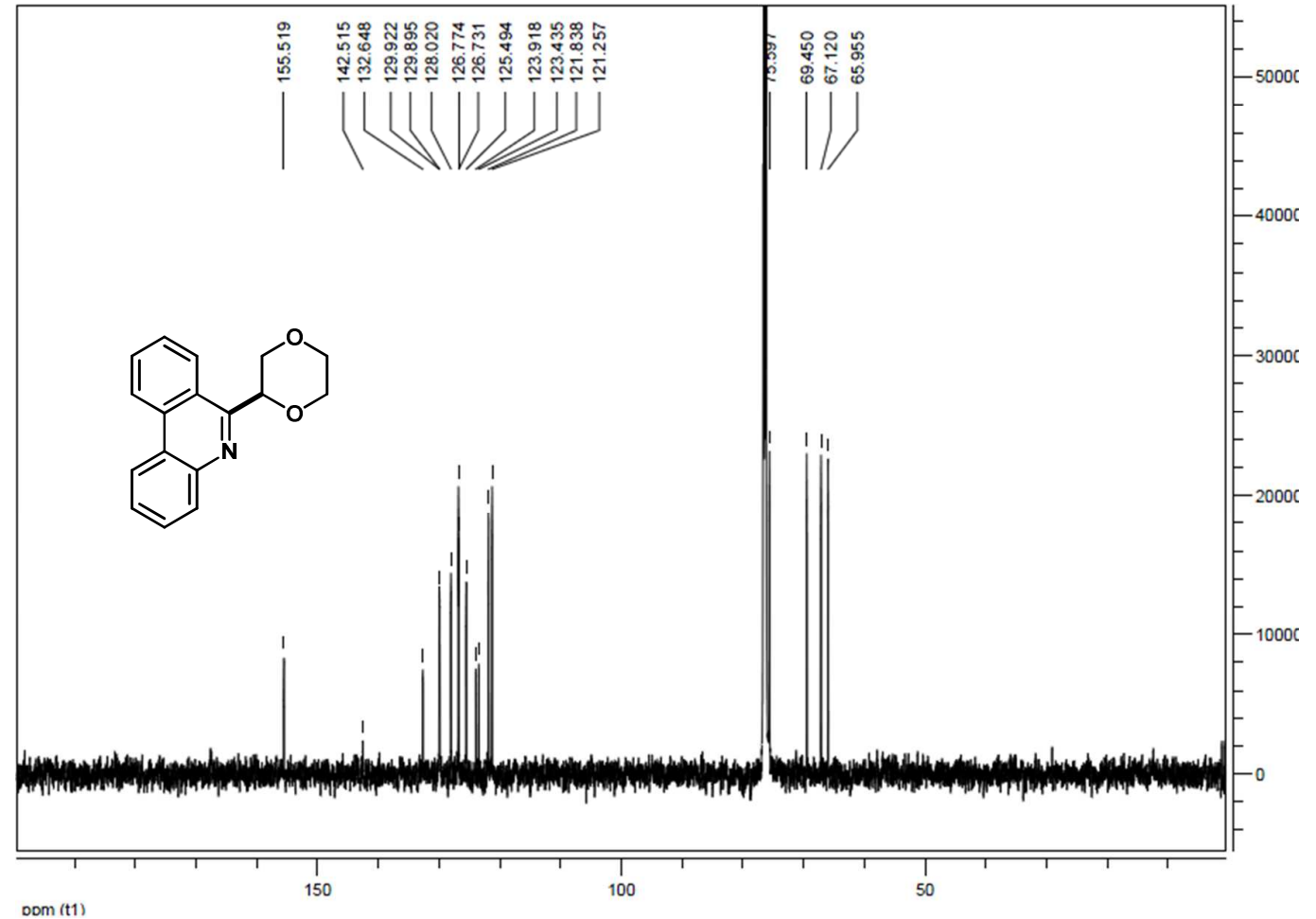


6-(tetrahydro-2H-pyran-2-yl)phenanthridine (4e)

\section{Proton NMR $\left(\mathrm{CDCl}_{3}\right)$}

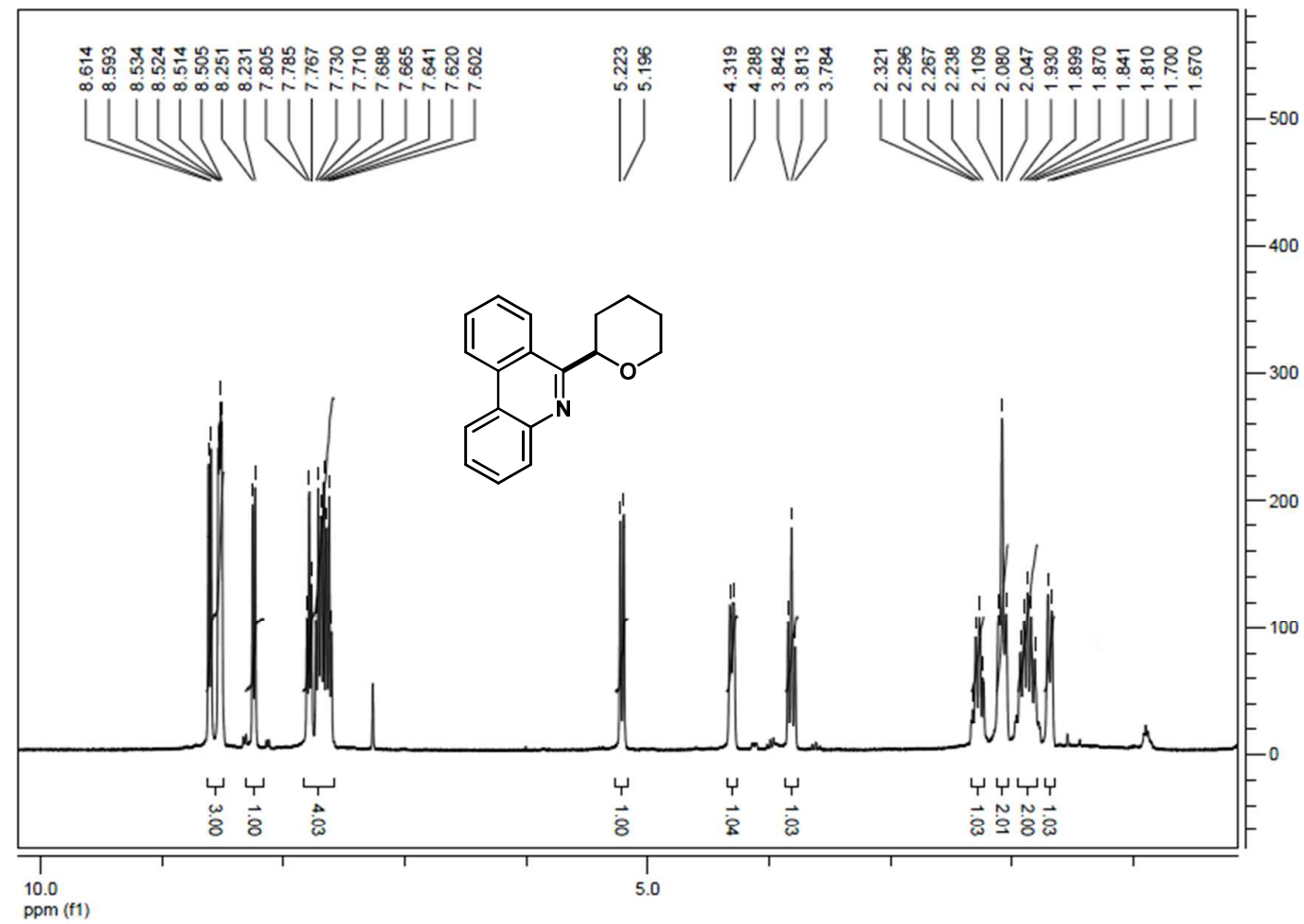

\section{Carbon NMR (CDCl $)_{3}$}

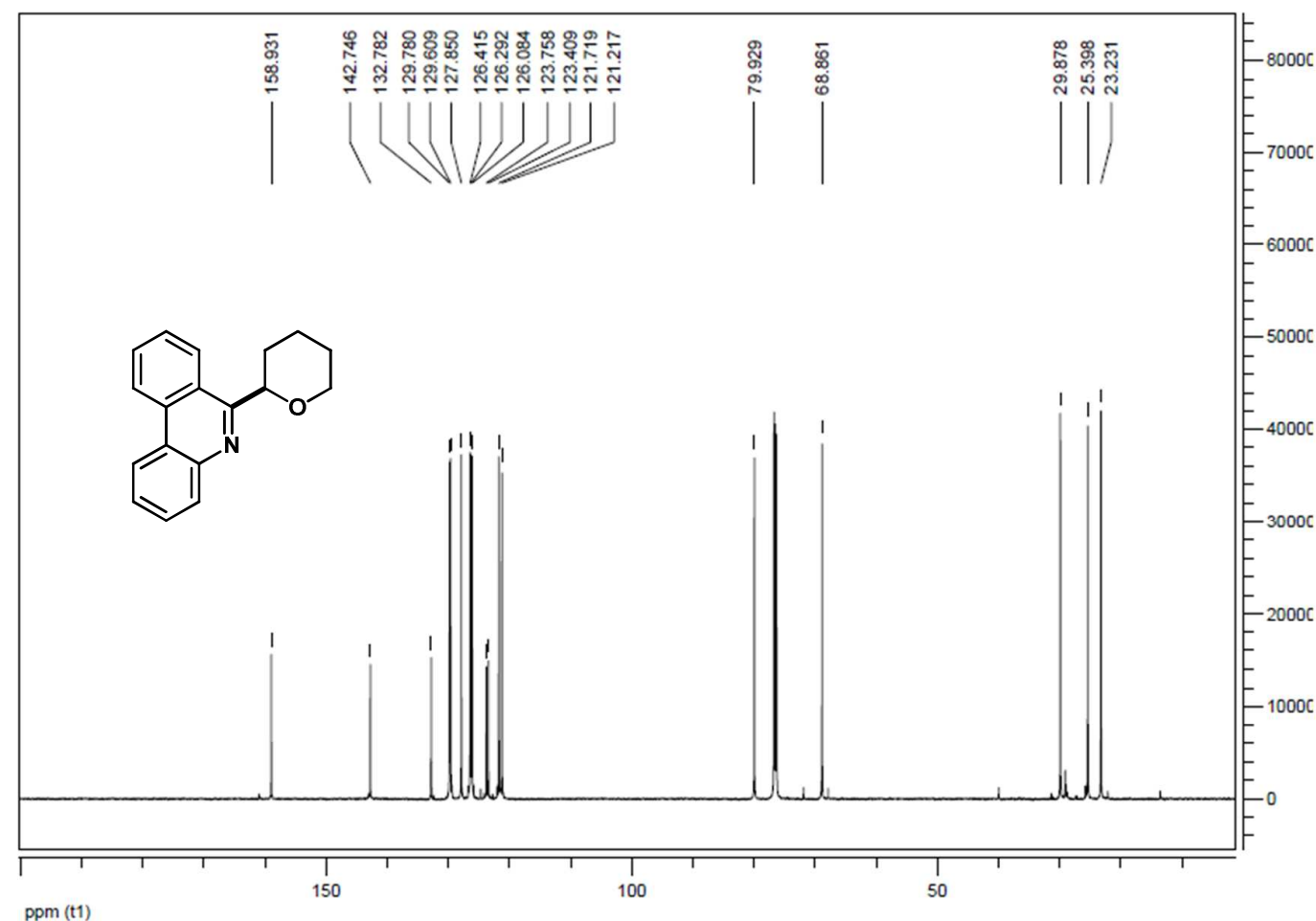


6-(2,3-dihydrobenzofuran-2-yl)phenanthridine (4f)

\section{Proton NMR $\left(\mathrm{CDCl}_{3}\right)$}

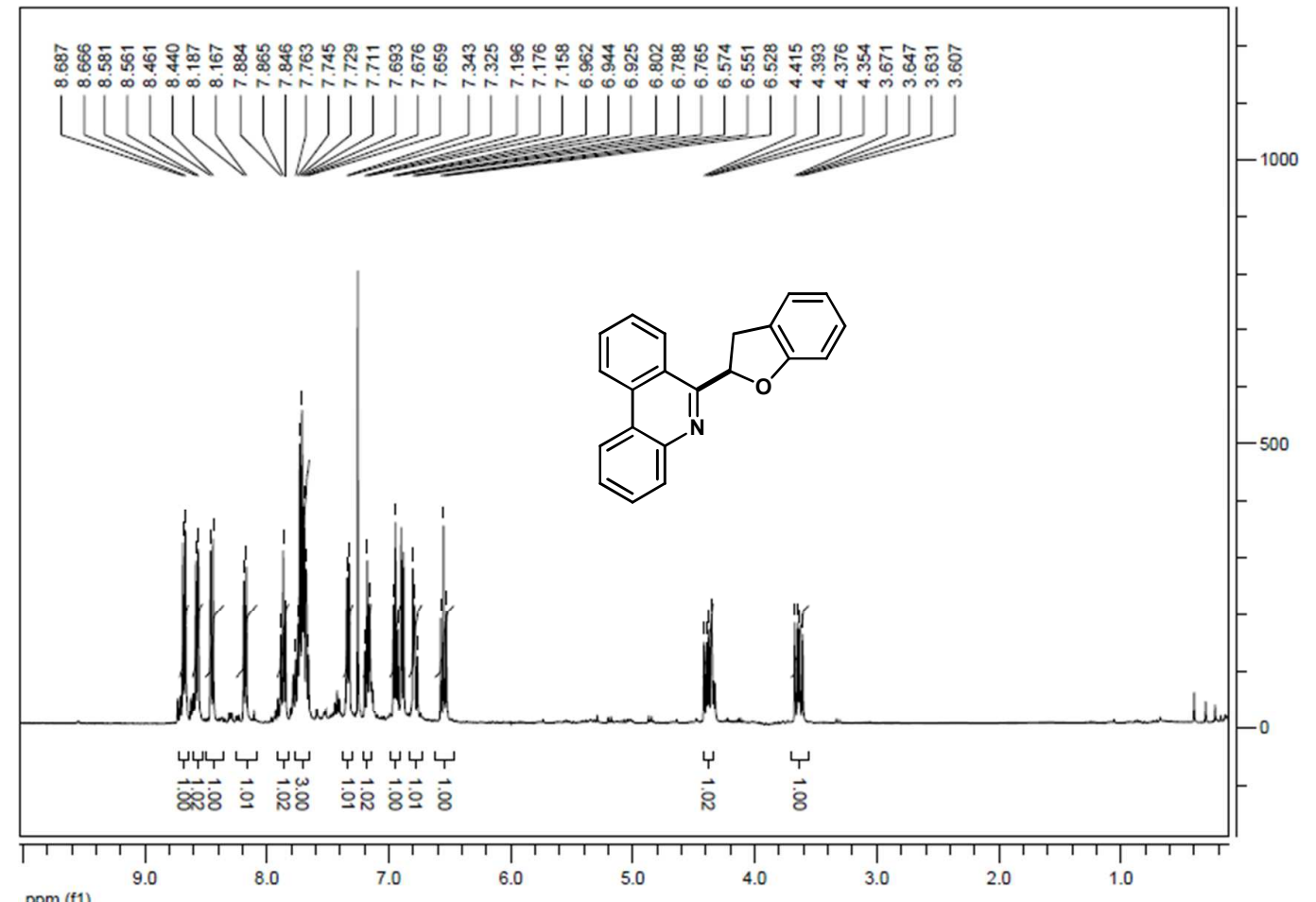

\section{Carbon NMR $\left(\mathrm{CDCl}_{3}\right)$}

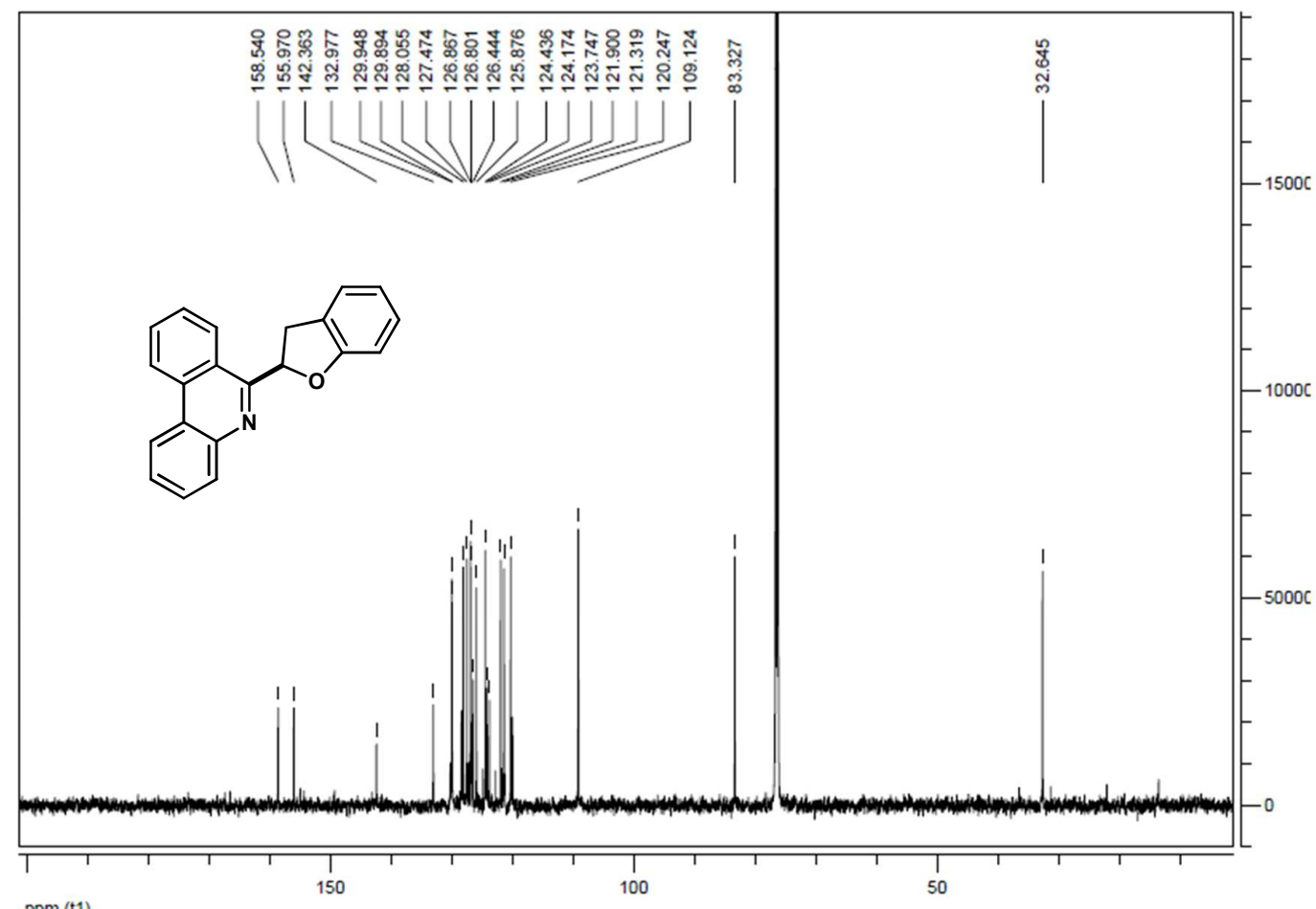

$\mathrm{ppm}(\mathrm{t} 1)$ 
6-(tetrahydro-2H-thiopyran-2-yl)phenanthridine (4g)

Proton NMR $\left(\mathrm{CDCl}_{3}\right)$

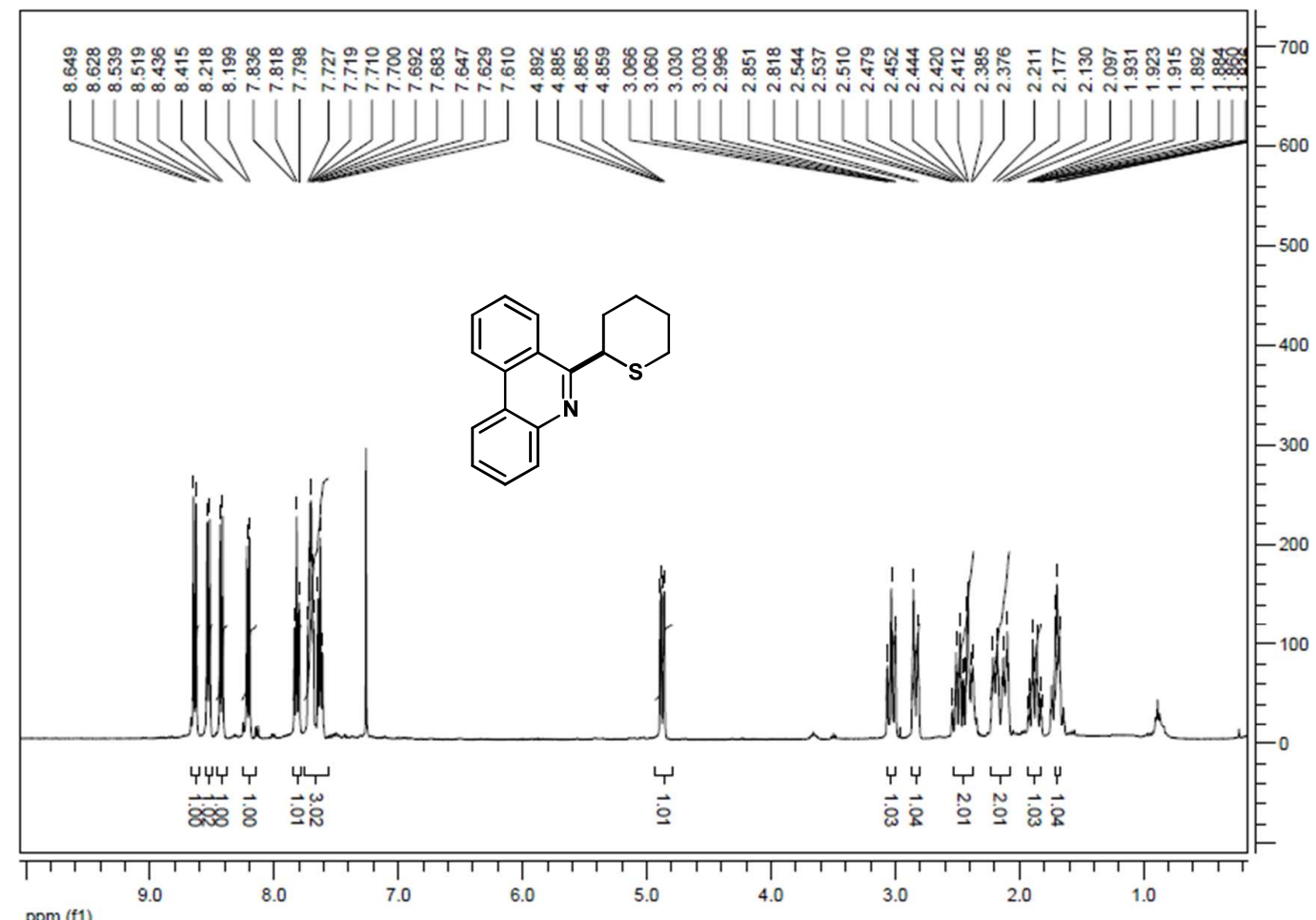

ppm (f1)

Carbon NMR (CDCl $\left.)_{3}\right)$

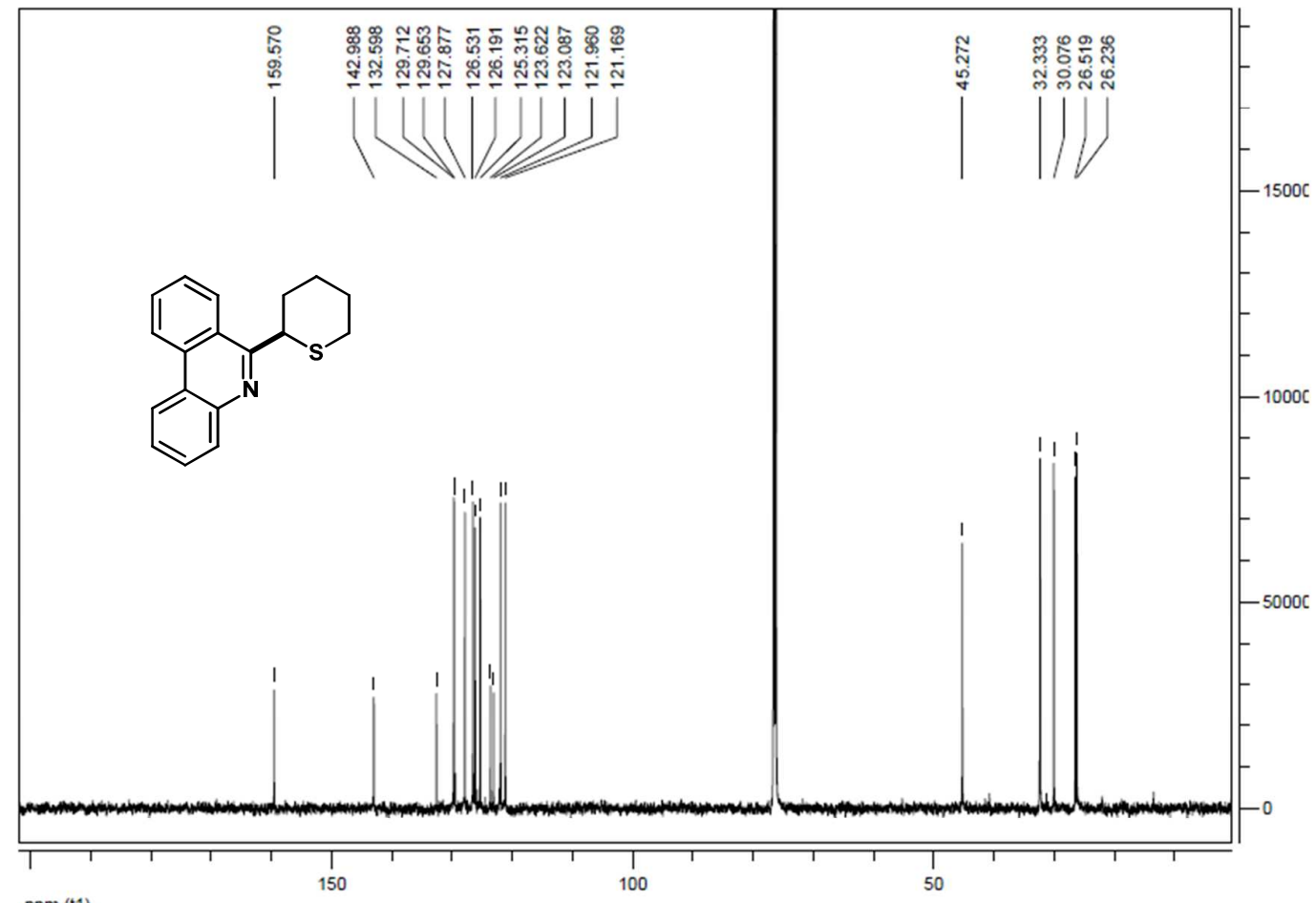

ppm (t1) 
$N$-methyl- $N$-(phenanthridin-6-ylmethyl)acetamide (4h)

Proton NMR $\left(\mathrm{CDCl}_{3}\right)$

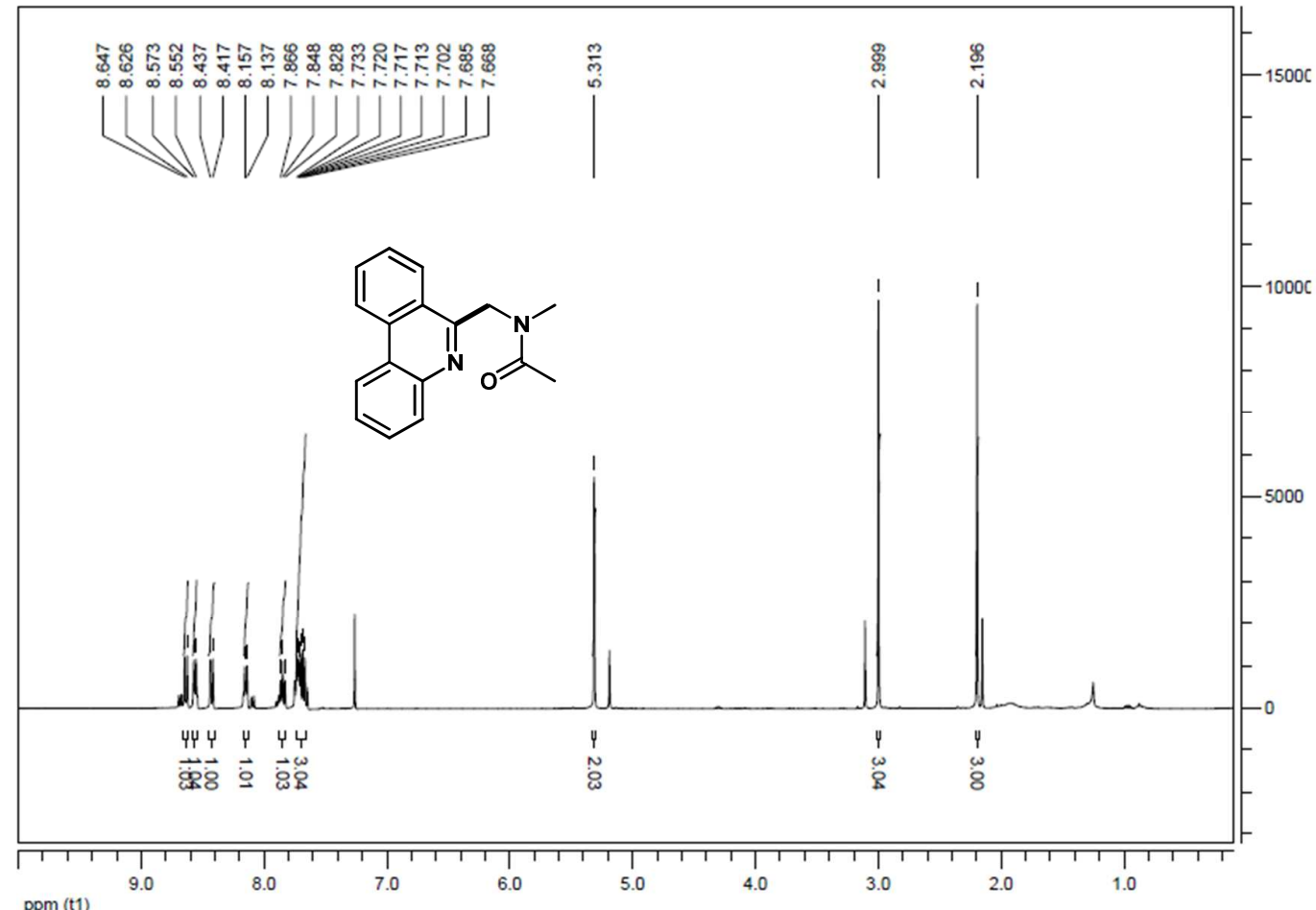

Carbon NMR (CDCl $\left.)_{3}\right)$

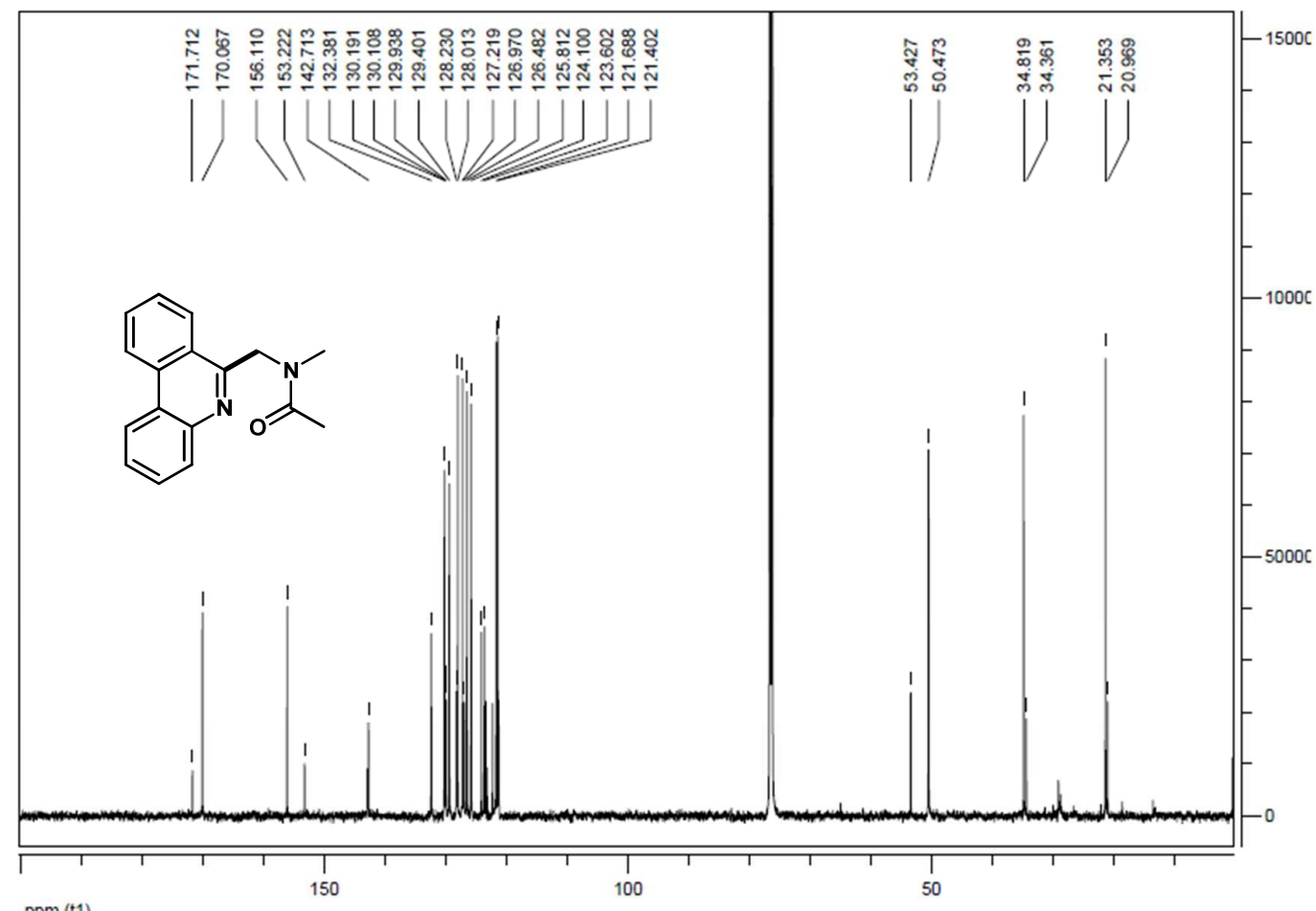

ppm (t1) 